\title{
Prospects for observing and localizing gravitational-wave transients with Advanced LIGO, Advanced Virgo and KAGRA
}

\author{
Abbott, B. P. et al. (KAGRA Collaboration, LIGO \\ Scientific Collaboration and Virgo Collaboration)*
}

Received: 11 September 2017 / Accepted: 7 February 2018 / Published online: 26 April 2018

(C) The Author(s) 2018

\begin{abstract}
We present possible observing scenarios for the Advanced LIGO, Advanced Virgo and KAGRA gravitational-wave detectors over the next decade, with the intention of providing information to the astronomy community to facilitate planning for multi-messenger astronomy with gravitational waves. We estimate the sensitivity of the network to transient gravitational-wave signals, and study the capability of the network to determine the sky location of the source. We report our findings for gravitationalwave transients, with particular focus on gravitational-wave signals from the inspiral of binary neutron star systems, which are the most promising targets for multi-messenger astronomy. The ability to localize the sources of the detected signals depends on the geographical distribution of the detectors and their relative sensitivity, and $90 \%$ credible regions can be as large as thousands of square degrees when only two sensitive detectors are operational. Determining the sky position of a significant fraction of detected signals to areas of 5-20 deg ${ }^{2}$ requires at least three detectors of sensitivity within a factor of $\sim 2$ of each other and with a broad frequency bandwidth. When all detectors, including KAGRA and the third LIGO detector in India, reach design sensitivity, a significant fraction of gravitational-wave signals will be localized to a few square degrees by gravitational-wave observations alone.
\end{abstract}

This article is a revised version of https://doi.org/10.1007/1rr-2016-1

Change summary: Major revision, updated and expanded.

Change details: Several updates to the document have been made. The most significant changes are the inclusion of details regarding KAGRA, and results from O1 and O2, including GW170817, the first detection with an unambiguous multi-messenger counterpart. The key differences are outlined in an appendix.

(KAGRA Collaboration, LIGO Scientific Collaboration and Virgo Collaboration) lvc.publications@ligo.org

*The full author list and affiliations are given at the end of paper. 
Keywords Gravitational waves - Gravitational-wave detectors - Electromagnetic counterparts · Data analysis

\section{Contents}

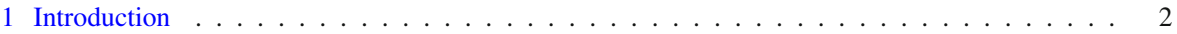

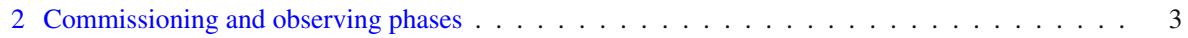

2.1 Commissioning and observing roadmap . . . . . . . . . . . . . . . . . . . . 4

2.2 Past and envisioned observing schedule . . . . . . . . . . . . . . . . 7

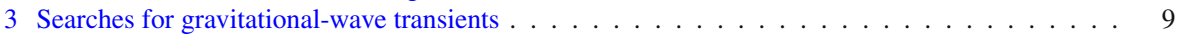

3.1 Detection and false alarm rates . . . . . . . . . . . . . . . . . . . . . . . 12

3.2 Localization . . . . . . . . . . . . . . . . . . . . . . . . . 15

3.2.1 Localization of binary neutron star coalescences . . . . . . . . . . . . . . . . . . 17

3.2 .2 Localization of bursts . . . . . . . . . . . . . . . . . . . . . . . . . . . . . . . . . . . . . . . .

4 Observing scenarios . . . . . . . . . . . . . . . . . . . . . . 22

$4.12015-2016$ run $(\mathrm{O} 1):$ aLIGO . . . . . . . . . . . . . . . . . . . . . 24

$4.22016-2017$ run $(\mathrm{O} 2)$ : aLIGO joined by AdV . . . . . . . . . . . . . . . . . . . . . 25

4.3 2018-2019 run (O3): aLIGO 120-170 Mpc, AdV 65-85 Mpc . . . . . . . . . . . . . . . 28

$4.42020+$ runs: aLIGO 190 Mpc, AdV 65-125 Mpc . . . . . . . . . . . . . . . . . . . . . 28

4.5 2024+ runs: aLIGO (including LIGO-India) $190 \mathrm{Mpc}$, AdV $125 \mathrm{Mpc}$, KAGRA $140 \mathrm{Mpc}$. . 28

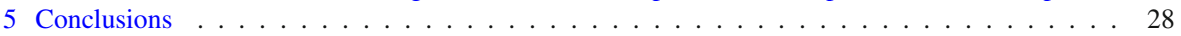

A Changes between versions . . . . . . . . . . . . . . . . . . . . 30

A.1 Updates to detector commissioning . . . . . . . . . . . . . . . . . . 30

A.2 Updates to data analysis . . . . . . . . . . . . . . . . . . . 31

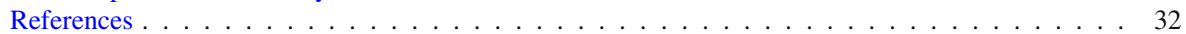

\section{Introduction}

Advanced LIGO (aLIGO; Harry 2010; Aasi et al. 2015a), Advanced Virgo (AdV; Acernese et al. 2009; Accadia et al. 2012; Acernese et al. 2015) and KAGRA (Somiya 2012; Aso et al. 2013) are kilometer-scale gravitational-wave (GW) detectors that are sensitive to GWs with frequencies of $\sim 20-2000 \mathrm{~Hz}^{1}{ }^{1}$ The era of GW astronomy began with the detection of GW150914 (Abbott et al. 2016j), a signal from the coalescence of a binary black hole (BBH); the first confirmed multi-messenger counterpart to a GW observation came with GW170817 (Abbott et al. 2017i), a signal from a binary neutron star (BNS) coalescence which was accompanied by detections across the electromagnetic spectrum (Abbott et al. 2017k). In this article, we describe the currently projected schedule, sensitivity, and sky-localization accuracy for the GWdetector network. We discuss the past and future planned sequence of observing runs (designated $\mathrm{O} 1, \mathrm{O} 2, \mathrm{O} 3$, etc.) and the prospects for multi-messenger astronomy.

The purpose of this article is to provide information to the astronomy community to assist in the formulation of plans for forthcoming $\mathrm{GW}$ observations. In particular, we intend this article to provide the information required for assessing the features of programs for joint observation of $\mathrm{GW}$ events using electromagnetic, neutrino, or other

\footnotetext{
${ }^{1}$ LIGO is short for Laser Interferometer Gravitational-Wave Observatory. KAGRA is named after the Japanese word for traditional sacred music and dance for the gods kagura; the name has a secondary meaning as an abbreviation for KAmioka GRavitational-wave Antenna. Virgo is not an acronym, and is not written in all caps.
} 
facilities (e.g., Abbott et al. 2016i; Adrian-Martinez et al. 2016; Albert et al. 2017c; Abbott et al. 2017k).

The full science of ground-based GW detectors is broad (Abbott et al. 2016j), and is not covered in this article. We concentrate solely on candidate GW transient signals. We place particular emphasis on the coalescence of BNS systems, which are the GW source for which electromagnetic follow-up is most promising (Metzger and Berger 2012; Patricelli et al. 2016; Paschalidis 2017; Rosswog et al. 2017; Metzger 2017). However, we also mention BBHs, as they are the most commonly detected source (Abbott et al. 2016c, 2017f). No electromagnetic emission is expected for vacuum BBH mergers (Centrella et al. 2010), but is possible if there is surrounding material (Schnittman 2013), for example, remnants of mass lost from the parent star (Perna et al. 2016; Janiuk et al. 2017) or if the binary was embedded in a circumbinary disc or a common envelope (Bartos et al. 2017; Woosley 2016; Stone et al. 2017). For more general introductory articles on GW generation, detection and astrophysics, we point readers to Blanchet (2014), Pitkin et al. (2011), Sathyaprakash and Schutz (2009).

Although our collaborations have amassed a great deal of experience with GW detectors and analysis, it is still difficult to make predictions for both improvements in search methods and for the rate of progress for detectors which are not yet fully installed or operational. The scenarios of detector sensitivity evolution and observing times given here should not be considered as fixed or firm commitments.

As the detectors' construction and commissioning progress, we intend to release updated versions of this article. This is the third version of the article, written to coincide with the close of the second observing run $(\mathrm{O} 2)$ of the advanced-detector era. Changes with respect to the previous version (Aasi et al. 2016) are given in Appendix A. Progress has been made in the commissioning of the detectors. We include projections for KAGRA for the first time; we also include results from the first observing run (O1) and currently available results from $\mathrm{O} 2$.

\section{Commissioning and observing phases}

We divide the development of the GW observatories into three components:

Construction includes the installation and testing of the detectors. This phase ends with acceptance of the detectors. Acceptance means that the interferometers can lock for periods of hours: light is resonant in the arms of the interferometer with no guaranteed $G W$ sensitivity. Construction incorporates several short engineering runs with no astrophysical output as the detectors progress towards acceptance. The aLIGO construction project ended in March 2015. The construction of AdV was completed at the end of 2016. KAGRA will be operational in its full configuration by early 2019 .

Commissioning improves the detectors' performance with the goal of reaching the design sensitivity. Engineering runs in the commissioning phase allow us to understand our detectors and analyses in an observational mode; these are not intended to produce astrophysical results, but that does not preclude the possi- 
bility of this happening. ${ }^{2}$ Rather than proceeding directly to design sensitivity before making astrophysical observations, commissioning is interleaved with observing runs.

Observing runs begin when the detectors have reached (and can stably maintain) a significantly improved sensitivity compared with previous operation. Observing runs will produce astrophysical results, direct detections from some GW sources and upper limits on the rates or energetics of others. During the first two observing runs (O1 and $\mathrm{O} 2)$, exchange of $\mathrm{GW}$ candidates with partners outside the LIGO Scientific Collaboration (LSC) and the Virgo Collaboration was governed by memoranda of understanding (MOUs; Abadie et al. 2012d; Aasi et al. 2013b). From the start of the third observing run (O3), all GW event candidates identified with high confidence will be released immediately to the full astronomical community. KAGRA will become a part of the global network with full data sharing, once sensitivities comparable with aLIGO and AdV are achieved.

The progress in sensitivity as a function of time will influence the duration of the observing runs that we plan at any stage. Commissioning is a complex process which involves both scheduled improvements to the detectors and tackling unexpected new problems. While our experience makes us cautiously optimistic regarding the schedule for the advanced detectors, we are targeting an order of magnitude improvement in sensitivity relative to the previous generation of detectors over a wider frequency band. Consequently, it is not possible to make concrete predictions for sensitivity or duty cycle as a function of time. We can, however, use our experience as a guide to plausible scenarios for the detector operational states that will allow us to reach the desired sensitivity. Unexpected problems could slow down the commissioning, but there is also the possibility that progress may happen faster than predicted here. The schedule of commissioning phases and observation runs will be driven by a costbenefit analysis of the time required to make significant sensitivity improvements. More information on event rates could also change the schedule and duration of runs.

In Sect. 2.1, we present the commissioning plans for the aLIGO, AdV and KAGRA detectors. A summary of expected observing runs is in Sect. 2.2.

\subsection{Commissioning and observing roadmap}

The anticipated strain sensitivity evolution for aLIGO, AdV and KAGRA is shown in Fig. 1. As a standard figure of merit for detector sensitivity, we use the range, the volume- and orientation-averaged distance at which a compact binary coalescence consisting of a particular mass gives a matched filter signal-to-noise ratio (SNR) of 8 in a single detector (Finn and Chernoff 1993). We define $V_{z}$ as the orientationaveraged spacetime volume surveyed per unit detector time; for a population with a constant comoving source-frame rate density, $V_{z}$ multiplied by the rate density gives the detection rate of those sources by the particular detector. We define the range $R$

\footnotetext{
2 The detection of GW150914 occurred in the engineering run ER8 immediately preceding the formal start of $\mathrm{O} 1$.
} 

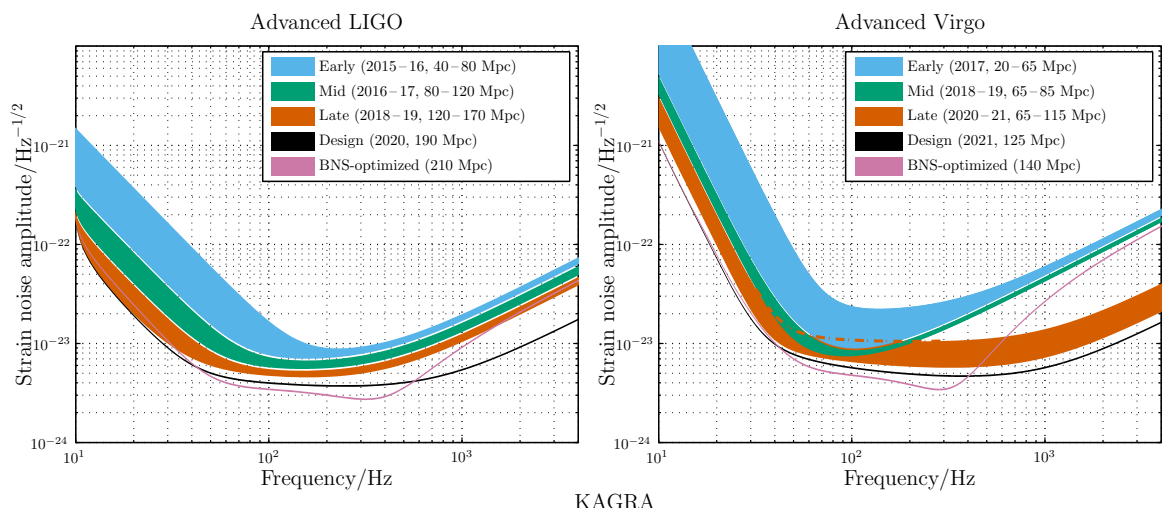

KAGRA

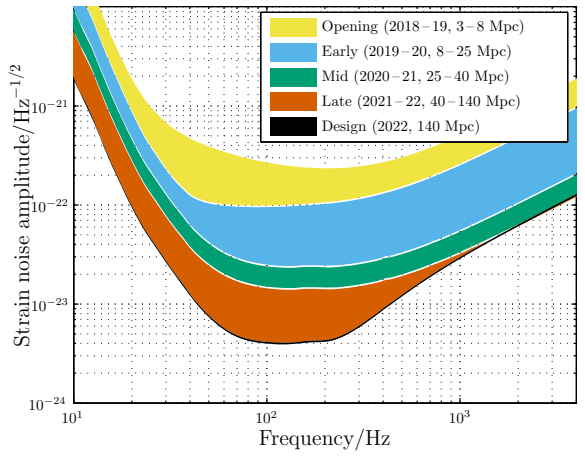

Fig. 1 Regions of aLIGO (top left), AdV (top right) and KAGRA (bottom) target strain sensitivities as a function of frequency. The binary neutron star (BNS) range, the average distance to which these signals could be detected, is given in megaparsec. Current notions of the progression of sensitivity are given for early, mid and late commissioning phases, as well as the design sensitivity target and the BNS-optimized sensitivity. While both dates and sensitivity curves are subject to change, the overall progression represents our best current estimates

as the distance for which $(4 \pi / 3) R^{3}=V_{z}$. In Table 1 we present values of $R$ for different detector networks and binary sources. For further insight into the range, and a discussion of additional quantities such as the median and average distances to sources, please see Chen et al. (2017). The BNS ranges, assuming two $1.4 M_{\odot}$ neutron stars, for the various stages of the expected evolution are provided in Fig. 1, and the BNS and BBH ranges are quoted in Table 1.

There are currently two operational aLIGO detectors (Aasi et al. 2015a). The original plan called for three identical 4-km interferometers, two at Hanford (H1 and H2) and one at Livingston (L1). In 2011, the LIGO Lab and IndIGO consortium in India proposed installing one of the aLIGO Hanford detectors (H2) at a new observatory in India (LIGO-India; Iyer et al. 2011). In early 2015, LIGO Laboratory placed the H2 interferometer in long-term storage for use in India. The Government of India granted in-principle approval to LIGO-India in February 2016.

The first observations with aLIGO have been made. O1 formally began 18 September 2015 and ended 12 January 2016; however, data from the surrounding engineering periods were of sufficient quality to be included in the analysis, and hence the first 
Table 1 Plausible target detector sensitivities

\begin{tabular}{|c|c|c|c|c|c|c|}
\hline & \multicolumn{2}{|l|}{ LIGO } & \multicolumn{2}{|l|}{ Virgo } & \multicolumn{2}{|l|}{ KAGRA } \\
\hline & $\begin{array}{l}\mathrm{BNS} \\
\text { range/Mpc }\end{array}$ & $\begin{array}{l}\mathrm{BBH} \\
\text { range/Mpc }\end{array}$ & $\begin{array}{l}\mathrm{BNS} \\
\text { range/Mpc }\end{array}$ & $\begin{array}{l}\mathrm{BBH} \\
\text { range/Mpc }\end{array}$ & $\begin{array}{l}\mathrm{BNS} \\
\text { range/Mpc }\end{array}$ & $\begin{array}{l}\mathrm{BBH} \\
\text { range/Mpc }\end{array}$ \\
\hline Early & $40-80$ & $415-775$ & $20-65$ & $220-615$ & $8-25$ & $8-250$ \\
\hline Mid & $80-120$ & $775-1110$ & $65-85$ & $615-790$ & $25-40$ & $250-405$ \\
\hline Late & $120-170$ & 1110-1490 & $65-115$ & $610-1030$ & $40-140$ & $405-1270$ \\
\hline Design & 190 & 1640 & 125 & 1130 & 140 & 1270 \\
\hline
\end{tabular}

The different phases match those in Fig. 1. We quote the range, the average distance to which a signal could be detected, for a $1.4 M_{\odot}+1.4 M_{\odot}$ binary neutron star (BNS) system and a $30 M_{\odot}+30 M_{\odot}$ binary black hole $(\mathrm{BBH})$ system

observations span 12 September 2015 to 19 January 2016. The run involved the H1 and L1 detectors; the detectors were not at full design sensitivity (Abbott et al. 2016f). We aimed for a BNS range of 40-80 Mpc for both instruments (see Fig. 1), and achieved a 60-80 Mpc range. Subsequent observing runs have increasing duration and sensitivity. $\mathrm{O} 2$ began 30 November 2016, transitioning from the preceding engineering run which began at the end of October, and ended 25 August 2017. The achieved sensitivity across the run was typically in the range 60-100 Mpc (Abbott et al. 2017f). Several improvements to the aLIGO detectors will be performed between $\mathrm{O} 2$ and $\mathrm{O} 3$, including further mitigation of technical noises, increase of laser power delivered to the interferometer, and installation of a squeezed vacuum source. Assuming that no unexpected obstacles are encountered, the aLIGO detectors are expected to achieve a $190 \mathrm{Mpc}$ BNS range by 2020. After the first observing runs, it might be desirable to optimize the detector sensitivity for a specific class of astrophysical signals, such as BNSs. The BNS range may then become $210 \mathrm{Mpc}$. The sensitivity for each of these stages is shown in Fig. 1.

The $\mathrm{H} 2$ detector will be installed in India once the LIGO-India Observatory is completed, and will be configured to be identical to the $\mathrm{H} 1$ and $\mathrm{L} 1$ detectors. We refer to the detector in this state as I1 (rather than H2). Operation at the same level as the $\mathrm{H} 1$ and L1 detectors is anticipated for no earlier than 2024.

The AdV interferometer (V1; Accadia et al. 2012) officially joined O2 on 1 August 2017. We aimed for an early step with sensitivity corresponding to a BNS range of 20-65 Mpc; however, AdV used steel wires to suspend the test masses, instead of fused silica fibers. This limited the highest possible BNS range in O2 to 40-60 Mpc; the BNS range achieved was 25-30 Mpc. Fused silica fibers will be reinstalled between $\mathrm{O} 2$ and O3. Other improvements such as reduction of technical noises, laser power increase and installation of a squeezed vacuum source will also be performed, bringing the AdV BNS range to 65-85 Mpc in 2018-2019. A configuration upgrade at this point will allow the range to increase to approximately $65-115 \mathrm{Mpc}$ in 2020 . The design sensitivity, with a BNS range of $125 \mathrm{Mpc}$, is anticipated circa 2021. The corresponding BNS-optimized range would be $140 \mathrm{Mpc}$. The sensitivity curves for the various AdV configurations are shown in Fig. 1. 
The KAGRA detector (K1; Somiya 2012; Aso et al. 2013) is located at the Kamioka underground site. The first operation of a detector in an initial configuration with a simple Michelson interferometer occurred in March 2016 (Akutsu et al. 2018). The detector is now being upgraded to its baseline design configuration. Initial operation at room temperature is expected in 2018. Subsequently, the detector will be cryogenically cooled to reduce thermal noise. Early cryogenic observations may come in 2019-2020 with a range of 8-25 Mpc. Since sensitivity will lag behind that of aLIGO and AdV, observing runs are planned to be short to allow commissioning to proceed as quickly as possible; longer observing runs may begin when the detector nears design sensitivity of $140 \mathrm{Mpc}$. The exact timing of observations has yet to be decided, but it is currently intended to have a three-month observing run in early 2020, and a six-month run at the start of 2021. The sensitivity curves for the various KAGRA commissioning stages are shown in Fig. 1.

GEO 600 (Lück et al. 2010; Dooley et al. 2016) will continue to operate as a GW detector beyond $\mathrm{O} 3$ as techniques for improving the sensitivity at high frequency are investigated (Affeldt et al. 2014). At its current sensitivity, it is unlikely to contribute to detections, but with a deliberate focus on high frequency narrow band sensitivity at a few kilohertz, GEO 600 may contribute to the understanding of BNS merger physics, as well as sky localization for such systems, by around 2021. In the meantime, it will continue observing with frequent commissioning and instrument science investigations related to detuned signal recycling and novel applications of squeezed light, as well as increasing the circulating power and levels of applied squeezing (Abadie et al. 2011; Grote et al. 2013; Aasi et al. 2013a; Brown et al. 2017).

Finally, further upgrades to the LIGO and Virgo detectors, within their existing facilities (e.g., Hild et al. 2012; Miller et al. 2015; Aasi et al. 2015c) as well as future third-generation observatories, for example, the Einstein Telescope (Punturo et al. 2010; Hild et al. 2011; Sathyaprakash et al. 2012) or Cosmic Explorer (Abbott et al. 2017d), are envisioned in the future. It is also possible that for some sources, there could be multiband gravitational-wave observations, where detection with a space-borne detector like the Laser Interferometer Space Antenna (LISA; AmaroSeoane et al. 2012, 2013) could provide early warning and sky localization (Sesana 2016), as well as additional information on system parameters (Vitale 2016), formation mechanisms (Nishizawa et al. 2016a, b; Breivik et al. 2016) and tests of general relativity (Barausse et al. 2016). These potential future developments are beyond the scope of this paper.

\subsection{Past and envisioned observing schedule}

Keeping in mind the important caveats about commissioning affecting the scheduling and length of observing runs, the following are plausible scenarios for the operation of the ground-based GW detector network over the next decade:

2015-2016 (O1) A four-month run (12 September 2015-19 January 2016) with the two-detector H1L1 network at early aLIGO sensitivity (60-80 Mpc BNS range). This is now complete. 


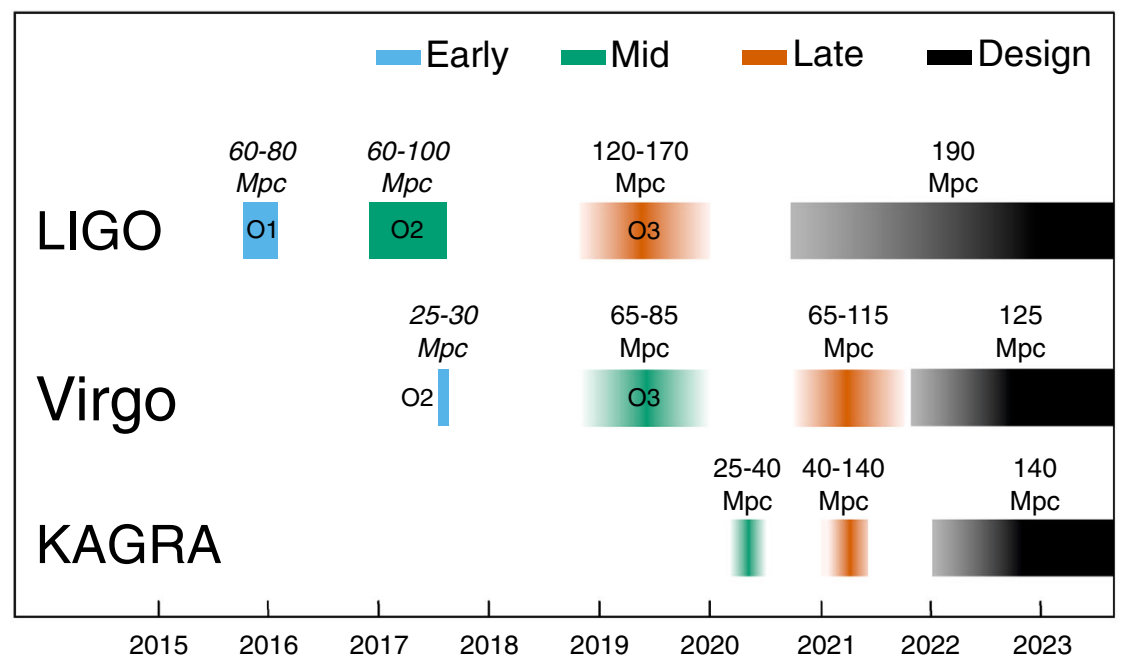

Fig. 2 The planned sensitivity evolution and observing runs of the aLIGO, AdV and KAGRA detectors over the coming years. The colored bars show the observing runs, with the expected sensitivities given by the data in Fig. 1 for future runs, and the achieved sensitivities in $\mathrm{O} 1$ and in $\mathrm{O} 2$. There is significant uncertainty in the start and end times of planned the observing runs, especially for those further in the future, and these could move forward or backwards relative to what is shown above. The plan is summarised in Sect. 2.2

2016-2017 (O2) A nine-month run with H1L1, joined by V1 for the final month. O2 began on 30 November 2016, with AdV joining 1 August 2017 and ended on 25 August 2017. The expected aLIGO range was $80-120 \mathrm{Mpc}$, and the achieved range was in the region of $60-100 \mathrm{Mpc}$; the expected AdV range was $20-65 \mathrm{Mpc}$, and the initial range was $25-30 \mathrm{Mpc}$.

2018-2019 (O3) A year-long run with H1L1 at 120-170 Mpc and with V1 at $65-85 \mathrm{Mpc}$ beginning about a year after the end of $\mathrm{O} 2$.

2020+ Three-detector network with H1L1 at full sensitivity of $190 \mathrm{Mpc}$ and V1 at $65-115 \mathrm{Mpc}$, later increasing to design sensitivity of $125 \mathrm{Mpc}$.

2024+ H1L1V1K1I1 network at full sensitivity (aLIGO at $190 \mathrm{Mpc}$, AdV at $125 \mathrm{Mpc}$ and KAGRA at $140 \mathrm{Mpc}$ ). Including more detectors improves sky localization (Klimenko et al. 2011; Veitch et al. 2012; Nissanke et al. 2013; Rodriguez et al. 2014; Pankow et al. 2018) as well as the fraction of coincident observational time. 2024 is the earliest time we imagine LIGO-India could be operational.

This timeline is summarized in Fig. 2; we do not include observing runs with LIGOIndia yet, as these are still to be decided. Additionally, GEO600 will continue observing, with frequent commissioning breaks, during this period. The observational implications of these scenarios are discussed in Sect. 4. 


\section{Searches for gravitational-wave transients}

Data from GW detectors are searched for many types of possible signals (Abbott et al. 2017j). Here we focus on signals from compact binary coalescence (CBCs) and on generic transient or burst signals. CBCs include BNS, neutron star-black hole (NS-BH) and BBH systems.

Observational results of searches for transient signals are reported in Abbott et al. (2016e, c, 1, 2017b, 2016q, 2017l,f, g, h, i). The O1 results include two clear detections GW150914 (Abbott et al. 2016k) and GW151226 (Abbott et al. 2016g), and a lower significance candidate LVT151012 (Abbott et al. 2016e, c). All three originate from BBH coalescences (Abbott et al. 2016m, c). No other transient sources have been identified in O1 (Abbott et al. 2016q, 2017b, m). The first results of O2 have been announced: GW170104 (Abbott et al. 2017f), GW170608 (Abbott et al. 2017g) and GW170814 (Abbott et al. 2017h) are from BBH coalescences, and GW170817 (Abbott et al. 2017i) is the first detection of a BNS coalescence.

Using our observation of GW170817, we calculate that the merger rate density has a $90 \%$ credible range of $320-4740 \mathrm{Gpc}^{-3} \mathrm{yr}^{-1}$ (Abbott et al. 2017i). This initial estimate assumes that neutron star masses are uniformly distributed between $1 M_{\odot}$ and $2 M_{\odot}$ and their dimensionless spins are less than 0.4. Compatible estimates for the merger rate were derived from the rate of electromagnetic transients similar to the counterpart of GW170817 (Siebert et al. 2017; Kasliwal et al. 2017; Smartt et al. 2017; Yang et al. 2017; Zhang et al. 2018). Complementary rate estimation based upon astrophysical population models and observations of Galactic BNS systems (e.g., Abadie et al. 2010b; Kim et al. 2013; Dominik et al. 2015; Vangioni et al. 2016; de Mink and Belczynski 2015; Eldridge et al. 2017; Belczynski et al. 2017; Kruckow et al. 2018) remains an active are of research.

While the rates per unit volume of $\mathrm{NS}-\mathrm{BH}$ and $\mathrm{BBH}$ mergers are expected to be lower than for BNSs, the distance to which they can be observed is larger. Consequently, the predicted observable rates are comparable (Abadie et al. 2010b; Rodriguez et al. 2015; Abbott et al. 2016b; Li et al. 2017). From our observations of $\mathrm{BBHs}$, we infer that their rate of mergers is in the range $\sim 1.2 \times 10^{-8}$ $2.13 \times 10^{-7} \mathrm{Mpc}^{-3} \mathrm{yr}^{-1}$ (Abbott et al. 2017f). The non-detection of NS-BHs in O1 allows us to place a $90 \%$ upper limit of the merger rate of $3.6 \times 10^{-6} \mathrm{Mpc}^{-3} \mathrm{yr}^{-1}$, assuming $1.4 M_{\odot}+5 M_{\odot}$ binaries with isotropically distributed spins (Abbott et al. 2016q); the upper limit on the rate decreases for higher mass black holes. Expected detection rates for other transient sources are lower and/or less well constrained.

For the purpose of detection, the gravitational waveform from the inspiral phase of a BNS coalescence is well modeled and matched filtering can be used to search for signals (Lindblom et al. 2008; Buonanno et al. 2009; Brown et al. 2012; Read et al. 2013; Abbott et al. 2016e; Harry et al. 2016). For systems containing black holes, or in which the component spin is significant, uncertainties in the waveform model can reduce the sensitivity of the search (Nitz et al. 2013; Harry et al. 2014; Dal Canton et al. 2015; Taracchini et al. 2014; Pan et al. 2014; Schmidt et al. 2015; Khan et al. 2016; Bustillo et al. 2017).

Searches for bursts make few assumptions on the signal morphology, using timefrequency decompositions to identify statistically significant excess-power transients 
in the data. Burst searches generally perform best for short-duration signals $(\lesssim 1 \mathrm{~s})$, although search development remains an area of active research (e.g., Klimenko et al. 2008; Sutton et al. 2010; Chassande-Mottin et al. 2010; Thrane et al. 2011; Adams et al. 2013; Thrane and Coughlin 2013; Cornish and Littenberg 2015; Thrane et al. 2015; Kanner et al. 2016); their astrophysical targets include core-collapse supernovae, magnetar flares, BBH coalescences, cosmic string cusps, and, possibly, as-yet-unknown systems.

In the era of advanced detectors, we are searching in near real-time for $\mathrm{CBC}$ and burst signals for the purpose of rapidly identifying event candidates. A prompt notice of a potential GW transient can enable follow-up observations in the electromagnetic spectrum.

A first follow-up program including low-latency analysis, event candidate selection, position reconstruction and the sending of alerts to several observing partners (optical, X-ray, and radio) was implemented and exercised during the 2009-2010 LIGO-Virgo science run (Abadie et al. 2012c, b; Evans et al. 2012). Latencies of less than $1 \mathrm{~h}$ were achieved.

In the present follow-up program, the LSC and Virgo distribute the times and sky localizations using the Gamma-ray Coordinates Network (GCN) system, widely used in the astronomical community for the multiwavelength follow-up of gamma-ray bursts. ${ }^{3}$ Messages are sent as machine-readable GCN Notices and as prose GCN Circulars, and astronomers communicate the results of observations using GCN Circulars. A shared infrastructure, including a database of results, allows observing partners to announce, coordinate and visualize the coverage of their observations. ${ }^{4}$

Prior to O1, 74 teams signed MOUs to participate in the electromagnetic follow-up program, and for the first event candidate later confirmed as GW150914, 63 were operational and covered the full electromagnetic spectrum (Abbott et al. 2016i,n). For the first observations with the advanced detectors, thorough checks were performed before alerts were released, resulting in latencies much greater than $1 \mathrm{~h}$. We expect this latency to be improved in the future as we gain experience with the instruments, and aim for automatic alerts being sent out with only a few minutes latency; continued checks may lead to retractions of some of these low-latency alerts. In the case of GW150914, 25 teams responded to the GW alert and operated ground- and space-based instruments spanning 19 orders of magnitude in electromagnetic wavelength (Soares-Santos et al. 2016; Annis et al. 2016; Connaughton et al. 2016; Ackermann et al. 2016; Savchenko et al. 2016; Kasliwal et al. 2016; Palliyaguru et al. 2016; Hurley et al. 2016; Morokuma et al. 2016; Copperwheat et al. 2016; Lipunov et al. 2017a; Kawai et al. 2017; Smartt et al. 2016b, b; Evans et al. 2016c; Díaz et al. 2016; Brocato et al. 2017). Analyses of archival data were also performed (Troja et al. 2016; Tavani et al. 2016). No significant electromagnetic counterpart and no afterglow emission was found in optical, ultravi-

\footnotetext{
3 Details of the GCN are available from gcn.gsfc.nasa.gov, and archives of messages for GW150 914 (gcn.gsfc.nasa.gov/other/G184098.gcn3), LVT151012 (gcn.gsfc.nasa.gov/other/G197392.gcn3), GW 151226 (gcn.gsfc.nasa.gov/other/G211117.gcn3), GW170104 (gcn.gsfc.nasa.gov/other/G268556.gcn3), GW170608 (gcn.gsfc.nasa.gov/other/G288732.gcn3), GW170814 (gcn.gsfc.nasa.gov/other/G297595. gcn3) and GW170817 (gcn.gsfc.nasa.gov/other/G298048.gcn3) are now publicly available.

4 More information about the follow-up program is available at gw-astronomy.org/wiki/LV_EM/TechInfo.
} 
olet, X-rays, or GeV gamma rays. The weak transient found in Fermi-GBM data $0.4 \mathrm{~s}$ after GW150914 (Connaughton et al. 2016; Bagoly et al. 2016) was not confirmed by other instruments like INTEGRAL SPI-ACS (Savchenko et al. 2016), AGILE (Tavani et al. 2016) or any other experiments of the InterPlanetary Network (Hurley et al. 2016). Models have been proposed to tentatively explain electromagnetic emission from BBHs, but there is no clear favorite as yet (Loeb 2016; Woosley 2016; Perna et al. 2016; Janiuk et al. 2017; Bartos et al. 2017; Stone et al. 2017; Li et al. 2016; Yamazaki et al. 2016; Ryan and MacFadyen 2017; Murase et al. 2016; Morsony et al. 2016; Dai et al. 2017; Lyutikov 2016; de Mink and King 2017). There was no significant neutrino emission temporally and spatially coincident with the event, and all detected neutrino candidates are consistent with the background (Adrian-Martinez et al. 2016; Gando et al. 2016; Aab et al. 2016; Abe et al. 2016; Agostini et al. 2017).

Similar follow-up campaigns have been performed for subsequent BBHs. ${ }^{3}$ LVT151012 was only identified in an offline search (Abbott et al. 2016e), as an online CBC search for BBHs was not running at the time. Nevertheless, some searching of archival data has been done, and no confident electromagnetic counterpart has been found (Racusin et al. 2017). GW151226 was identified by a low-latency online search (Abbott et al. 2016g) and a variety of teams followed up; no electromagnetic counterpart has been reported (Cowperthwaite et al. 2016; Smartt et al. 2016a; Copperwheat et al. 2016; Racusin et al. 2017; Evans et al. 2016b; Adriani et al. 2016; Palliyaguru et al. 2016; Yoshida et al. 2017; Serino et al. 2017; Brocato et al. 2017). No significant neutrino counterpart was found in coincidence with either LVT151012 or GW151226 (Albert et al. 2017c; Gando et al. 2016; Aab et al. 2016; Abe et al. 2016; Agostini et al. 2017). For GW170104 (Abbott et al. 2017f), a Circular with initial localization was sent in under $7 \mathrm{~h}$; no confirmed electromagnetic or neutrino counterpart has been reported (Bhalerao et al. 2017; Verrecchia et al. 2017; Corsi et al. 2017; Stalder et al. 2017; Goldstein et al. 2017b; Agostini et al. 2017; Savchenko et al. 2017b; Albert et al. 2017a). ${ }^{3}$ Identification of GW170608 was delayed due to maintenance work being undertaken at Hanford at the time of the event (Abbott et al. $2017 \mathrm{~g}$ ), but a Circular was still issued within 14 h. ${ }^{3}$ No conclusive counterpart has yet been reported. GW170814 was the first event to be confidently detected by Virgo and the inclusion of the third detector significantly improved the localization for this event (Abbott et al. 2017h). A Circular and initial sky localization was issued in under 2 h. ${ }^{3}$ No counterpart has been reported so far (Arcavi et al. 2017b). A lack of counterparts is unsurprising given our current understanding of BBHs.

GW170817 was the first GW transient for which a firm electromagnetic counterpart was discovered (Abbott et al. 2017k). On 2017 August 17 12:41:06 UTC, FermiGBM triggered on a short GRB, GRB 170817A (Goldstein et al. 2017a), and a GCN was sent after $14 \mathrm{~s}$. About 6 min later, a GW trigger was identified; the signal was consistent with a BNS coalescence (Abbott et al. 2017i) occurring $\sim 1.7$ s before GRB 170817A (Abbott et al. 2017e), and a GCN was issued at 13:08:16 UTC. ${ }^{3}$ A threedetector GW localization was issued within $11 \mathrm{~h}$ of detection. An extensive observing campaign was launched, leading to the discovery of the bright transient AT 2017gfo by the One-Meter, Two-Hemisphere team with the 1-m Swope Telescope (Coulter et al. 2017), and confirmed by other teams within an hour (Soares-Santos et al. 2017; Valenti et al. 2017; Arcavi et al. 2017a; Tanvir et al. 2017; Lipunov et al. 2017b). Subsequent 
infrared-ultraviolet observations targeted the transient and measured its brightness and spectrum, revealing a red-ward evolution (e.g., Villar et al. 2017). X-ray (Troja et al. 2017; Margutti et al. 2017; Haggard et al. 2017; Ruan et al. 2018; Pooley et al. 2017; D'Avanzo et al. 2018) and radio (Hallinan et al. 2017; Alexander et al. 2017; Mooley et al. 2018) afterglows were discovered at the position of the transient after $\sim 9$ day and $\sim 16$ day respectively; these were later joined by observation of the optical afterglow (Lyman et al. 2018; Margutti et al. 2018). Follow-up observations did not reveal any neutrino (Albert et al. 2017b) or high-energy gamma-ray (Abdalla et al. 2017) emission at the position of AT 2017gfo. These multimessenger observations support the hypothesis that GW170817 came from a BNS coalescence, which was the source of the short GRB 170817A (Goldstein et al. 2017a; Savchenko et al. 2017a) and a kilonova powered by the radioactive decay of r-process nuclei produced in the collision (Pian et al. 2017; McCully et al. 2017; Smartt et al. 2017; Chornock et al. 2017; Nicholl et al. 2017; Shappee et al. 2017).

The multimessenger observations for GW170817 allow for different studies ranging from astrophysics to fundamental physics and cosmology. The GW and gamma-ray data show that the BNS coalescence and the short gamma-ray are associated (Abbott et al. 2017e). The time delay of $\sim 1.7$ s between GW170817 and GRB 170817A places a constraint on the size and bulk Lorentz factor of the emitting region; furthermore, this delay constrains the difference between the speed of light and the speed of gravity, places new bounds on the violation of Lorentz invariance, and tests the equivalence principle by constraining the Shapiro delay between gravitational and electromagnetic radiation (Abbott et al. 2017e). These results limit the range of potential viable alternative theories of gravity (e.g., Creminelli and Vernizzi 2017; Sakstein and Jain 2017; Ezquiaga and Zumalacárregui 2017; Boran et al. 2018; Baker et al. 2017; Arai and Nishizawa 2017). GWs can be used as standard sirens for cosmological measurements (Schutz 1986). Combining the inferred GW distance with the redshift of the host galaxy NGC 4993, it was possible to infer the Hubble constant (Abbott et al. 2017a); the result is in agreement with the values determined from supernova (Riess et al. 2016) and cosmic microwave background measurements (Ade et al. 2016).

Increased detection confidence, improved sky localization, and identification of host galaxy and redshift are just some of the benefits of joint GW-electromagnetic observations. With this in mind, we focus on two points of particular relevance for follow-up of GW events: the source localization afforded by a GW network as well as the relationship between signal significance, or false alarm rate (FAR), and source localization.

\subsection{Detection and false alarm rates}

Detection pipelines search the data looking for signal-like features. Candidate triggers flagged by a pipeline are assigned a detection statistic to quantify how signal-like they are. For CBC searches, this involves matching a bank of waveform templates (Sathyaprakash and Dhurandhar 1991; Owen 1996; Owen and Sathyaprakash 1999; Babak et al. 2006; Cokelaer 2007; Prix 2007; Harry et al. 2009; Ajith et al. 
2014; Brown et al. 2012; Capano et al. 2016; Dal Canton and Harry 2017) to the data (Abbott et al. 2016e, c); for burst searches, requirements on waveform morphology are relaxed, but coherence of the signal in multiple detectors is required (Abbott et al. 20161, 2017b). The detection statistic is used to rank candidates; we assess significance by comparing results with those from an estimated background distribution of noise triggers. It is difficult to theoretically model the behaviour of non-Gaussian noise, and therefore the distribution must be estimated from the data (Abadie et al. 2010a; Babak et al. 2013; Abadie et al. 2012a; Abbott et al. 2016a; Capano et al. 2017; Messick et al. 2017; Abbott et al. 2016e, c, 1, 2017b; Nitz et al. 2017). From the background noise distribution we can map a value of the detection statistic to a FAR, the expected rate of triggers with detection statistics equal to or greater than that value, assuming that the data contain no signals. While each pipeline has its own detection statistic, they all compute a FAR, making it easy to compare results. The FAR, combined with the observation time, may then be used to calculate a $p$ value, the probability of there being at least one noise trigger with a FAR this small or smaller in the observed time. ${ }^{5}$ As the FAR or $p$ value of a trigger decreases, it becomes more significant, and more likely to be a genuine astrophysical signal.

The rate of noise triggers above a given SNR depends critically upon the data quality of the advanced detectors; non-stationary transients or glitches (Aasi et al. 2012, 2015b; Abbott et al. 2016d) produce an elevated background of loud triggers. Over 200,000 auxiliary channels record data on instrumental and environmental conditions (Effler et al. 2015; Abbott et al. 2016d). These channels act as witnesses of disturbances that may couple into the GW channel. An intensive study of the data quality is used to veto stretches of acquired data ranging from seconds to hours in duration. When a significant problem with the data is identified or a known instrumental issue affects the searches' background, the contaminated data are removed from the analysis data set. Our experience to date is that this removes a small percentage of the data; for example, in $\mathrm{O} 1$ vetoes removed less than $5 \%$ of the coincident data from the $\mathrm{CBC}$ analysis, with a single intermittent instrumental problem accounting for $4.65 \%$ of that total (Abbott et al. 2016d, c, 2017b, 2018). For low-mass CBC searches, the waveforms are well modeled, and signal consistency tests reduce the background significantly (Allen 2005; Cannon et al. 2015; Usman et al. 2016). For burst sources which are not well modeled, or which spend only a short time in the detectors' sensitive band, it is more difficult to distinguish between the signal and a glitch, and so a reduction of the FAR comes at a higher cost in terms of reduced detection efficiency (or live-time if more vetoes are used).

Search pipelines are run both online, analysing data as soon as they are available in order to provide low-latency alerts of interesting triggers, and offline, taking advantage

\footnotetext{
5 The $p$ value is distinct from the probability that a trigger is not a real astrophysical GW signal. The $p$ value assumes that the data contain no signals, whereas the probability of there being a GW must include the hypothesis that there is an astrophysical signal. To calculate the probability that a trigger is a real signal (or not) requires an extra layer of inference, folding in both our knowledge of the distribution of triggers, assumptions about the signal distribution (such as that sources are uniformly distributed in volume), and knowledge and assumptions about the merger rate per unit volume for a class of sources. A method for doing this is described in Abbott et al. (2016p, o, c).
} 

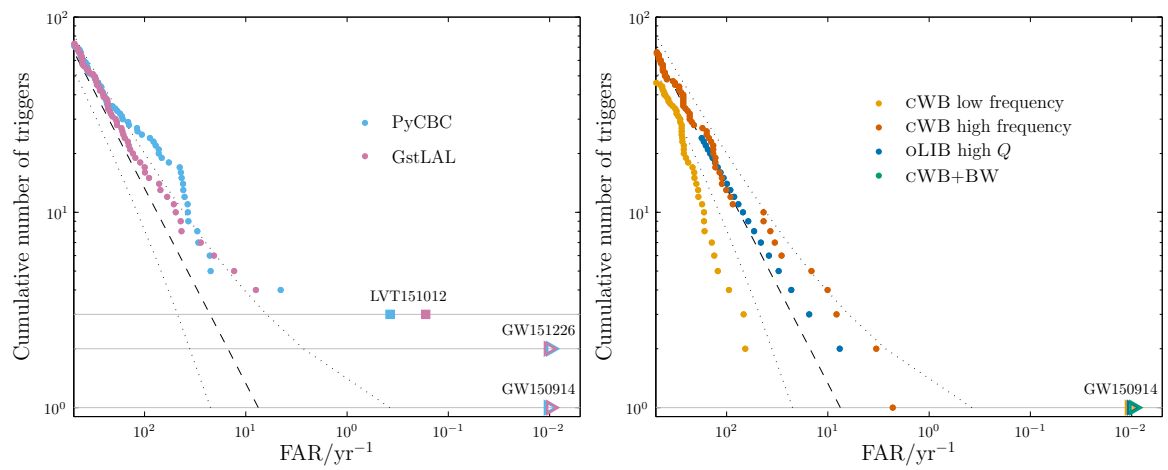

Fig. 3 Offline transient search results for the first observing run: the cumulative number of triggers with false alarm rates (FARs) smaller than the abscissa value. The dashed line shows the expected noise-only distribution, and the dotted lines show the $90 \%$ confidence interval assuming no signals. Potential signals are identified by having smaller FARs than expected. The plots are truncated at a minimum FAR of $10^{-2} \mathrm{yr}^{-1}$. Left: Compact binary coalescence search results (Abbott et al. 2016c, q). We show results from two search algorithms, GstLAL (Cannon et al. 2012; Privitera et al. 2014; Messick et al. 2017) and PyCBC (Dal Canton et al. 2014; Usman et al. 2016). The most significant triggers for both are LVT151012, GW151226 and GW150914; GW150914 and GW151226 have FARs less than $10^{-2} \mathrm{yr}^{-1}$. Right: Burst search results (Abbott et al. 2017b). We show results from three search algorithms, COHERENT WAVE BURST (CWB; Klimenko et al. 2016, 2008), Omicron-LALINFERENCEBURST (OLIB; Lynch et al. 2017) and BAYESWAVE follow-up of CWB (CWB+BW; Kanner et al. 2016). All three found GW150914 (the only CWB trigger above the BAYESWAVE follow-up threshold) with a FAR less than $10^{-2} \mathrm{yr}^{-1}$. GW151226 and LVT151012 fall below the burst search's detection threshold

of improved calibration of the data and additional information regarding data quality. In Fig. 3, we show the offline transient search results for $01 .^{6}$

For $\mathrm{CBC}$, we show the cumulative number of triggers at a given FAR for two pipelines: PyCBC (Dal Canton et al. 2014; Usman et al. 2016) and GstLAL (Cannon et al. 2012; Privitera et al. 2014; Messick et al. 2017). In the $\mathrm{O} 2$ analysis, PyCBC uses an improved detection statistic (Nitz et al. 2017). GW150914, LVT151012 and GW151226 are visible in both the GstLAL and PyCBC results (Abbott et al. 2016e, c) shown in Fig. 3.

For bursts, we show distributions for COHERENT WAVE BURST (CWB; Klimenko et al. 2016, 2008), Omicron-LALINFERENCEBURST (OLIB; Lynch et al. 2017) and BAYESWAVE (Cornish and Littenberg 2015; Littenberg and Cornish 2015). The CWB analysis is split into two frequency bands, above and below $1024 \mathrm{~Hz}$. The OLIB search is split into two bins, based upon the quality factor $Q$ of the sine-Gaussian it uses to model the signal; no triggers were identified by the low- $Q$ search. BAYESWAVE is run as a follow-up to triggers identified by CWB (Kanner et al. 2016), and hence is not completely independent. GW150914 was identified by all three search algorithms (Abbott et al. 20161, 2017b).

For CBC signals, we conservatively estimate that a network SNR threshold of $\rho_{c} \simeq 12$ is required for a FAR below $\sim 10^{-2} \mathrm{yr}^{-1}$ in the advanced-detector era (Abadie

\footnotetext{
6 Strain data from published probable events in $\mathrm{O} 1$ and $\mathrm{O} 2$ are publicly available from the LIGO Open Science Center losc.ligo.org (Vallisneri et al. 2015). Full data from O1 are also available, and data from other observing runs will be added in the future.
} 
et al. 2012f; Aasi et al. 2016; Berry et al. 2015). A combined SNR of 12 corresponds to a single-detector SNR of 8.5 in each of two detectors, or 7 in each of three detectors (assuming an orientation and sky location for which the detectors have equal sensitivity). The exact threshold will depend upon data quality in each observing run as well as the mass of the source; in O1, we found that the threshold SNR was lower, around 10 .

\subsection{Localization}

Following the detection of a GW transient posterior probability distributions for the position are constructed following a Bayesian framework (Veitch et al. 2015; Cornish and Littenberg 2015; Singer and Price 2016; Abbott et al. 2016m), with information for the sky localization coming from the time of arrival, plus the phase and amplitude of the GW.

An intuitive understanding of localization can be gained by considering triangulation using the observed time delays between sites (Fairhurst 2009, 2011). The effective single-site timing accuracy is approximately

$$
\sigma_{t}=\frac{1}{2 \pi \rho \sigma_{f}},
$$

where $\rho$ is the SNR in the given detector and $\sigma_{f}$ is the effective bandwidth of the signal in the detector, typically of order $100 \mathrm{~Hz}$. Thus a typical timing accuracy is on the order of $10^{-4} \mathrm{~s}$ (about $1 / 100$ of the $10 \mathrm{~ms}$ light travel time between sites). This sets the localization scale. The simple model of Eq. (1) ignores many other relevant issues such as information from the signal amplitudes and phases across the detector network, uncertainty in the emitted gravitational waveform, instrumental calibration accuracies, and correlation of sky location with other binary parameters (Fairhurst 2009; Vitale and Zanolin 2011; Vitale et al. 2012; Nissanke et al. 2011; Veitch et al. 2012; Nissanke et al. 2013; Singer et al. 2014; Berry et al. 2015; Singer and Price 2016; Fairhurst 2017). While many of these affect the measurement of the time of arrival in individual detectors, such factors are largely common between two similar detectors, so the time difference between the two detectors is relatively uncorrelated with these additional parameters.

The timing-triangulation approach underestimates how well a source can be localized, since it does not include all the relevant information. Its predictions can be improved by introducing the requirement of phase and amplitude consistency between detectors (Grover et al. 2014; Fairhurst 2017): it always performs poorly for a twodetector network, but, with the inclusion of phase coherence, can provide an estimate for the average performance of a three-detector network (Berry et al. 2015).

Source localization using only timing for a two-site network yields an annulus on the sky; see Fig. 4. Additional information such as signal amplitude and phase, and precession effects resolve this to only parts of the annulus, but even then sources will only be localized to regions of hundreds to thousands of square degrees (Singer et al. 2014; Berry et al. 2015). An example of a two-detector BNS localization is shown in 


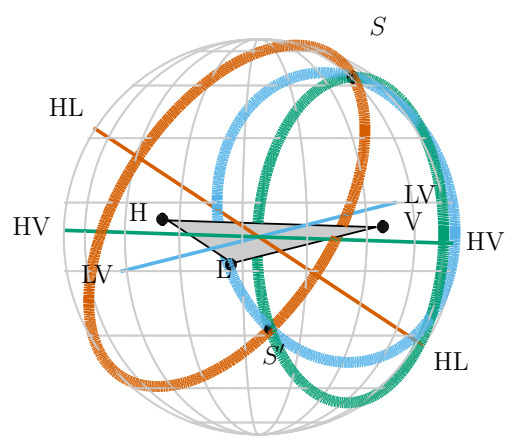

Fig. 4 Source localization by timing triangulation for the aLIGO-AdV network. The locations of the three detectors are indicated by black dots, with LIGO Hanford labeled H, LIGO Livingston as L, and Virgo as $\mathrm{V}$. The locus of constant time delay (with associated timing uncertainty) between two detectors forms an annulus on the sky concentric about the baseline between the two sites (labeled by the two detectors). For three detectors, these annuli may intersect in two locations. One is centered on the true source direction $(S)$, while the other $\left(S^{\prime}\right)$ is its mirror image with respect to the geometrical plane passing through the three sites. For four or more detectors there is a unique intersection region of all of the annuli. Image adapted from Chatterji et al. (2006)

Fig. 5. The posterior probability distribution is primarily distributed along a ring, but this ring is broken, such that there are clear maxima.

For three detectors, the time delays restrict the source to two sky regions which are mirror images with respect to the plane passing through the three sites. It is often possible to eliminate one of these regions by requiring consistent amplitudes in all detectors (Fairhurst 2017). For signals just above the detection threshold, this typically yields regions with areas of several tens to hundreds of square degrees. If there is significant difference in sensitivity between detectors, the source is less well localized and we may be left with the majority of the annulus on the sky determined by the two most sensitive detectors. With four or more detectors, timing information alone is sufficient to localize to a single sky region, and the additional baselines help to limit the region to under $10 \mathrm{deg}^{2}$ for some signals.

From Eq. (1), it follows that the linear size of the localization ellipse scales inversely with the SNR of the signal and the frequency bandwidth of the signal in the detector (Berry et al. 2015). For GWs that sweep across the band of the detector, such as CBC signals, the effective bandwidth is $\sim 100 \mathrm{~Hz}$, determined by the most sensitive frequencies of the detector. Higher mass CBC systems merge at lower frequencies and so have a smaller effective bandwidth. For burst transients, the bandwidth $\sigma_{f}$ depends on the specific signal. For example, GWs emitted by various processes in core-collapse supernovae are anticipated to have relatively large bandwidths, between $150 \mathrm{~Hz}$ and 500 Hz (Dimmelmeier et al. 2008; Ott 2009; Yakunin et al. 2010; Ott et al. 2011), largely independent of detector configuration. By contrast, the sky localization region for narrowband burst signals may consist of multiple disconnected regions and exhibit fringing features; see, for example, Klimenko et al. (2011), Abadie et al. (2012c), Essick et al. (2015). 
In addition to localizing sources on the sky, for CBC signals it is possible to provide distance estimates, since the waveform amplitude is inversely proportional to the luminosity distance (Veitch et al. 2015; Abbott et al. 2016m). Uncertainty in distance measurement is dominated by the degeneracy with the binary's inclination, which also determines the signal amplitude (Cutler and Flanagan 1994; Nissanke et al. 2010; Aasi et al. 2013c). The degeneracy could be broken by observing with more non co-aligned detectors (Veitch et al. 2012; Rodriguez et al. 2014), or if precession of the orbital plane is observed (Vecchio 2004; van der Sluys et al. 2008; Vitale et al. 2014), but this is not expected for slowly spinning BNS (Farr et al. 2016). Distance information can further aid the hunt for counterparts, particularly if the the localization can be used together with galaxy catalogs (Nissanke et al. 2013; Hanna et al. 2014; Fan et al. 2014; Blackburn et al. 2015; Singer et al. 2016a).

Some GW searches are triggered by electromagnetic observations, and in these cases some localization information is known a priori. For example, in GW searches triggered by gamma-ray bursts (Abadie et al. 2012e; Aasi et al. 2014c, b; Abbott et al. 20171), the triggering space-based telescope provides a localization. The coincident observation of GW170817 (Abbott et al. 2017k) and GRB 170817A (Goldstein et al. 2017a; Savchenko et al. 2017a) confirms that BNS mergers are a progenitor for short gamma-ray bursts (Abbott et al. 2017d), and therefore that gamma-ray bursts are interesting targets for triggered GW searches. Other possible targets for these externally-triggered GW searches could be electromagnetic or neutrino emission from Galactic core-collapse supernovae. It is therefore of great scientific value to have telescopes capable of observing the high-energy spectrum operating during the advanced-detector era (and beyond). Furthermore, the rapid identification of a GW counterpart to such a trigger could prompt further spectroscopic studies and longer, deeper follow-up in different wavelengths that may not always be done in response to gamma-ray bursts (cf. Abbott et al. 2017k). This is particularly important for gamma-ray bursts with larger sky localization uncertainties, such as those reported by the Fermi-GBM, which are not followed up as frequently as bursts reported by Swift or Fermi-LAT; in the case of GW170817, the LIGO-Virgo localization was tighter than the localization from Fermi-GBM and INTEGRAL (Abbott et al. 2017e; Goldstein et al. 2017a; Savchenko et al. 2017a) and also showed that the source was nearby $\left(40_{-14}^{+8} \mathrm{Mpc}\right.$; Abbott et al. 2017i), making it a prime target for further follow-up. All GW data are stored permanently, so that it is possible to perform retroactive analyses at any time.

\subsubsection{Localization of binary neutron star coalescences}

Providing prompt localizations for GW signals helps to maximise the chance that electromagnetic observatories can catch a counterpart. Localizations are produced at several different latencies, with updates coming from more computationally expensive algorithms that refine our understanding of the source.

For CBC signals, rapid localization is performed using BAYESTAR (Singer and Price 2016), a Bayesian parameter-estimation code that computes source location using output from the detection pipeline. It can produce sky localizations (as in Fig. 5) 
with latencies of only a few seconds. A similar approach to low-latency localization has been separately developed by Chen and Holz (2015), which find results consistent with BAYESTAR. BAYESTAR can also provide distance estimates (Singer et al. 2016a). These can be easily communicated as an additional component of the sky localization: for each line of sight, the distance posterior probability is approximated as a Gaussian multiplied by the distance squared (Singer et al. 2016a, b). ${ }^{7}$ Results from BAYESTAR are shared at low latency for prompt follow-up efforts.

At higher latency, CBC parameter estimation is performed using the stochastic sampling algorithms of LALINFERENCE (Veitch et al. 2015). LALINFERENCE constructs posterior probability distributions for system parameters, not just location like BAYESTAR, but also mass, orientation, etc. (Aasi et al. 2013c; Abbott et al. 2016m), by matching GW templates to the detector strain (Cutler and Flanagan 1994; Jaranowski and Królak 2012). Computing these waveforms is computationally expensive; this expense increases as the detectors' low-frequency sensitivity improves and waveforms must be computed down to lower frequencies. The quickest LALINFERENCE BNS follow-up is computed using waveforms that do not include the full effects of component spins (Singer et al. 2014; Berry et al. 2015; Abbott et al. 2017f), localizations can then be reported with latency of hours to a couple of days. Parameter estimation is then performed using more accurate waveform approximates, those that include fuller effects of spin precession and the effects of tidal distortions of neutron stars (Farr et al. 2016; Abbott et al. 2016h, 2017f, i). Provided that BNSs are slowly spinning (Mandel and O'Shaughnessy 2010), the restrictions on the spins should cause negligible difference between the mid-latency LALINFERENCE and the high-latency fully spinning LALINFERENCE localizations (Farr et al. 2016). Methods of reducing the computational cost are actively being investigated (e.g., Canizares et al. 2013; Pürrer 2014; Canizares et al. 2015; Smith et al. 2016; Vinciguerra et al. 2017).

Sky localization results from an astrophysically motivated population of BNS signals, assuming a detection threshold of a SNR of 12, are shown in Fig. 6 (Singer et al. 2014; Berry et al. 2015). Results are quantified using the $90 \%$ credible region $\mathrm{CR}_{0.9}$, the smallest area enclosing $90 \%$ of the total posterior probability, and the searched area $A_{*}$, the area of the smallest credible region that encompasses the true position (Sidery et al. 2014): $\mathrm{CR}_{0.9}$ gives the area of the sky that must be covered to expect a $90 \%$ chance of including the source location, and $A_{*}$ gives the area that would be viewed before the true location is found using the given sky localization. Results from both the low-latency BAYESTAR and mid-latency LALINFERENCE analyses are shown. These are discussed further in Sects. 4.1 and 4.2. The two-detector localizations are slightly poorer in $\mathrm{O} 2$ than in $\mathrm{O} 1$. This is because although the detectors improve in sensitivity at every frequency, with the assumed noise curves the BNS signal bandwidth is lower in O2 for a given SNR because of enhanced sensitivity at low frequencies (Singer et al. 2014). Sky localization improves with the expansion of the detector network (Schutz 2011; Klimenko et al. 2011; Veitch et al. 2012; Rodriguez et al. 2014; Gaebel and Veitch 2017; Pankow et al. 2018).

\footnotetext{
7 A data release of example three-dimension localizations in this format, constructed using results from BAYESTAR and LALINFERENCE for BNS signals, is available from dcc.ligo.org/P1500071/public/html.
} 

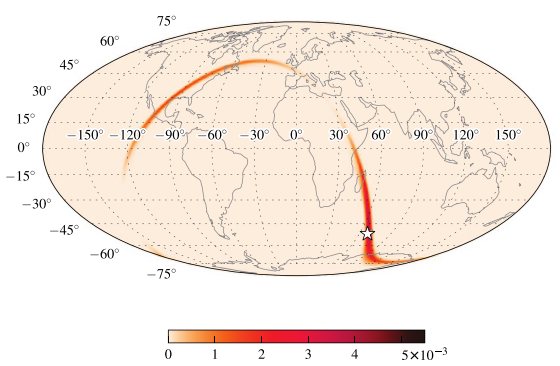

Posterior probability density $/ \mathrm{deg}^{-2}$
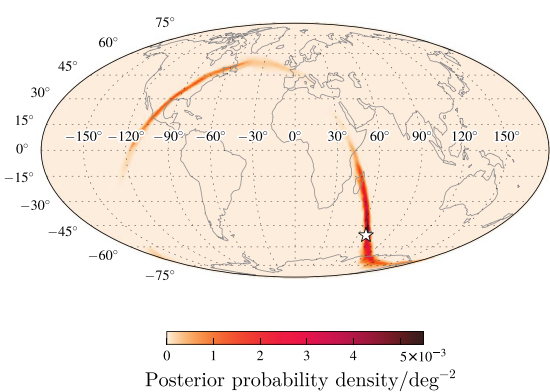

Fig. 5 Posterior probability density for sky location for an example simulated binary neutron star coalescence observed with a two-detector network. Left: Localization produced by the low-latency BAYESTAR code (Singer et al. 2014; Singer and Price 2016). Right: Localization produced by the higher-latency (neglecting spin) LALINFERENCE (Veitch et al. 2015), which also produces posterior estimates for other parameters. These algorithms are discussed in Sect. 3.2.1, and agreement between them shows that the low-latency localization is comparable to the one produced by the higher-latency pipelines. The star indicates the true source location. The source is at a distance of $266 \mathrm{Mpc}$ and has a network signal-to-noise ratio of $\rho_{c}=13.2 \mathrm{using}$ a noise curve appropriate for the first aLIGO run (O1, see Sect. 4.1). The plot is a Mollweide projection in geographic coordinates. Figure reproduced with permission from Berry et al. (2015), copyright by AAS; further mock sky localizations for the first two observing runs can be found at www.ligo.org/scientists/first2years/ for binary neutron star signals and www.ligo.org/scientists/burst-first2years/ for burst signals

The results in Fig. 6 show there is negligible difference between the low-latency BAYESTAR and the LALINFERENCE analyses if the BNS signal is loud enough to produce a trigger in all detectors. However, when the signal is sub-threshold in one, LALINFERENCE could give a more precise localization, as it still uses strain data from the non-triggered detector (Singer et al. 2014; Singer and Price 2016). In preparation for the start of joint three-detector observations in $\mathrm{O} 2$, the online $\mathrm{CBC}$ pipelines and BAYESTAR have been enhanced to capture and make use of sub-threshold signals in all detectors. Consequently, there should be negligible difference between the low-latency BAYESTAR and LALINFERENCE localizations even for events that register weakly in one or more detectors. This was the case for GW170817, where both analyses produced a $90 \%$ credible area of $\sim 30 \mathrm{deg}^{2}$ (Abbott et al. 2017k).

For the BNS signals from the sky-localization studies, the average fractional distance uncertainty, defined as the posterior standard deviation divided by the mean, is $\sim 0.25-0.30$ (Berry et al. 2015; Farr et al. 2016).

LALINFERENCE also has the ability to include the effects of the detectors' calibration uncertainty on parameter estimation (Abbott et al. 2016m,c). Calibration is refined as additional measurements are taken, hence sky localization can improve as uncertainty is reduced. Initial results for GW150914 assumed a calibration uncertainty of $10 \%$ for the amplitude of the GW strain and $10 \mathrm{deg}$ for its phase (Abbott et al. 2017c). Incorporating this calibration uncertainty into the analysis, the $90 \%$ credible area was $610 \mathrm{deg}^{2}$ (Abbott et al. 2016m). By the end of O1, the calibration uncertainty had been improved, such that the $90 \%$ credible area was $230 \mathrm{deg}^{2}$ (Abbott et al. 2016c). If the detectors were assumed to be perfectly calibrated, such that calibration uncertainty could be ignored, the $90 \%$ credible area would be $150 \mathrm{deg}^{2}$. Sky localization is particularly sensitive to calibration uncertainty and distance is less affected. 

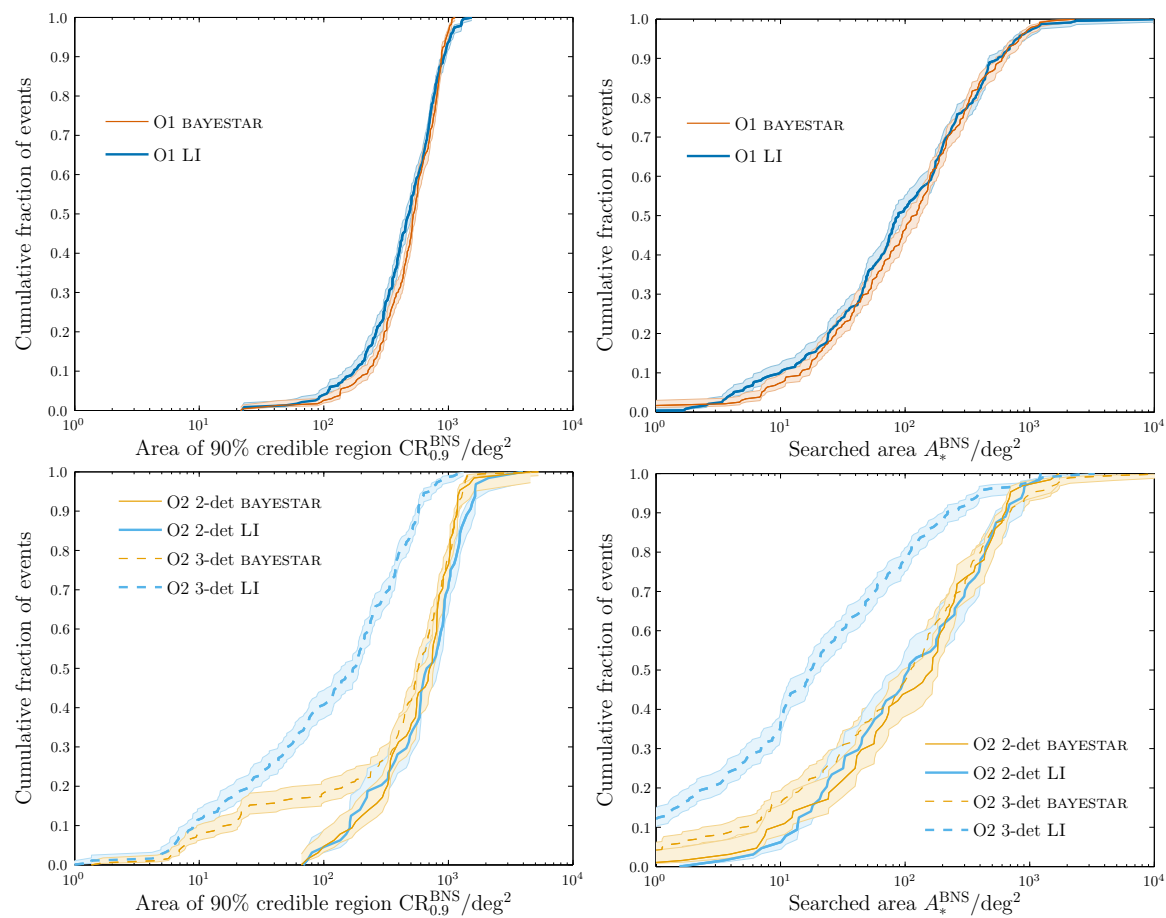

Fig. 6 Anticipated binary neutron star sky localization during the first two observing runs (top: O1, see Sect. 4.1; bottom: O2, see Sect. 4.2). Detector sensitivities were taken to be in the middle of the early (for aLIGO in $\mathrm{O} 1$ and AdV in O2) and mid (for aLIGO in O2) bands of Fig. 1. The plots show the cumulative fractions of events with sky-localization areas smaller than the abscissa value. Left: Sky area of $90 \%$ credible region $\mathrm{CR}_{0.9}^{\mathrm{BNS}}$, the (smallest) area enclosing $90 \%$ of the total posterior probability. Right: Searched area $A_{*}^{\mathrm{BNS}}$, the area of the smallest credible region containing the true position. Results are shown for the low-latency BAYESTAR (Singer and Price 2016) and higher-latency (neglecting spin) LALINFERENCE (LI; Veitch et al. 2015) codes. The $\mathrm{O} 2$ results are divided into those where two detectors (2-det) are operating in coincidence, and those where three detectors (3-det) are operating: assuming a duty cycle of 70-75\% for each instrument, the two-detector network would be operating for $42-44 \%$ of the total time and the three-detector network $34-42 \%$ of the time. The shaded areas indicate the $68 \%$ confidence intervals on the cumulative distributions. A detection threshold of a signal-to-noise ratio of 12 is used and results are taken from Berry et al. (2015), Singer et al. (2014)

For GW150914, the initial distance estimate was $410_{-180}^{+160} \mathrm{Mpc}$ (Abbott et al. 2016m), the estimate at the end of the run was $420_{-180}^{+150} \mathrm{Mpc}$, and the equivalent result without calibration uncertainty was $420_{-170}^{+140} \mathrm{Mpc}$ (Abbott et al. 2016c). The effects of calibration uncertainty depend upon the signal's SNR, bandwidth and position of the source relative to the detectors. For GW151226, LVT151012 and GW170104, there is negligible difference between the sky areas or distances with and without calibration uncertainty using the final calibration uncertainties (Abbott et al. 2016c, 2017f). 


\subsubsection{Localization of bursts}

Sky localizations are also produced for burst triggers and distributed for follow up. The lowest latency burst sky localizations are produced as part of the CWB detection pipeline (Klimenko et al. 2008, 2016). Sky localizations are produced using a constrained likelihood algorithm that coherently combines data from all the detectors. The CWB sky localizations are calculated with a latency of a few minutes; following detection, further parameter-estimation codes analyze the data.

At higher latency, burst signals are analyzed by LALINFERENCEBURST (LIB), a stochastic sampling algorithm similar to the LALINFERENCE code used to reconstruct CBC signals (Veitch et al. 2015), and BAYESWAVE, a reversible jump Markovchain Monte Carlo algorithm that models both signals and glitches (Cornish and Littenberg 2015). LIB uses sine-Gaussian waveforms (in place of the CBC templates used by LALINFERENCE), and can produce sky localizations in a few hours. BAYESWAVE uses a variable number of sine-Gaussian wavelets to model a signal and glitches while also fitting for the noise spectrum using BAYESLINE (Littenberg and Cornish 2015); it produces sky localizations with a latency of a few days.

The sky-localization performance of burst algorithms depends upon the type of signal. Studies of burst localization using BAYESWAVE in the first year of the advanceddetector era, and using CWB and LIB in the first two years have been completed in Bécsy et al. (2016) and Essick et al. (2015), respectively. These assumed sensitivities in the middle of the early band for aLIGO in O1, and in the middle of the mid band for aLIGO and the early band for AdV in O2. Sky localization was quantified by the searched area. For the CWB and LIB pipelines an approximate FAR threshold of $1 \mathrm{yr}^{-1}$ was used to select events; BAYESWAVE was run as a follow-up to triggers identified by CWB (Kanner et al. 2016), and sky localization was only performed on triggers also detected by BAYESWAVE (and not classified as noise or a glitch). The median localization was shown to be $\sim 100-200 \mathrm{deg}^{2}$ for a twodetector network in $\mathrm{O} 1$ and $\sim 60-110 \mathrm{deg}^{2}$ for a three-detector network in $\mathrm{O} 2$, with the localization and relative performance of the algorithms depending upon the waveform morphology. Results for Gaussian, sine-Gaussian, broadband whitenoise and BBH waveforms are shown in Fig. 7 (for the two-detector O1 network and the three-detector $\mathrm{O} 2$ network, cf. Fig. 6). The variety of waveform morphologies reflect the range of waveforms that could be detected in a burst search (Abadie et al. 2012c).

A high mass BBH, like GW150914 (Abbott et al. 2016k), could be detected by both burst and $\mathrm{CBC}$ analyses. In this case, we expect that the $\mathrm{CBC}$ localization, which makes use of the additional information available from constraining signals to match waveform templates, is more accurate than the burst localization (cf. Vitale et al. 2016). 

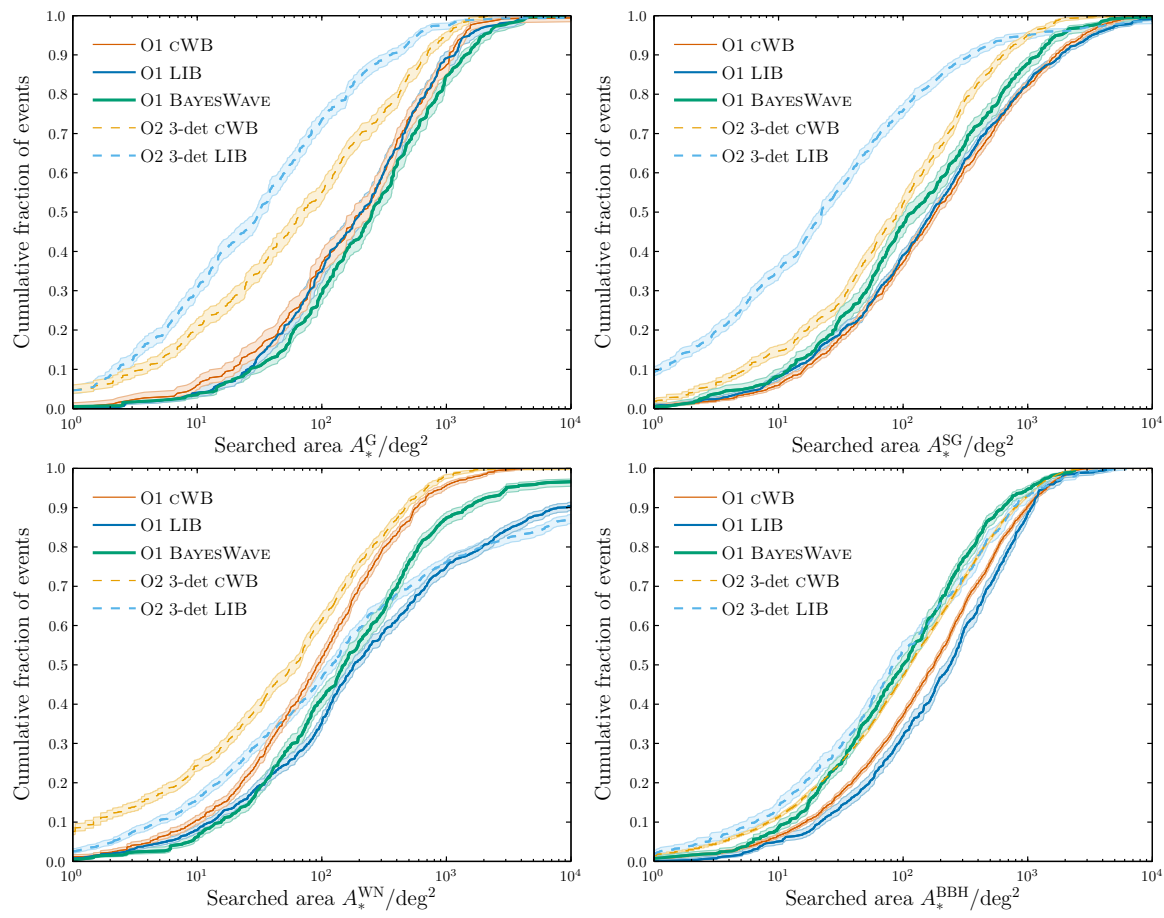

Fig. 7 Simulated sky localization for Gaussian (G; top left), sine-Gaussian (SG; top right), broadband white-noise (WN; bottom left) and binary black hole (BBH; bottom right) bursts during the first two observing runs (O1, see Sect. 4.1, and O2, see Sect. 4.2). The plots show the cumulative fractions of events with searched areas $A_{*}$ smaller than the abscissa value. Results are shown for the low-latency COHERENT WAVEBURST (CWB; Klimenko et al. 2005, 2008, 2016), and higher-latency LALINFERENCEBURST (LIB; Veitch et al. 2015) and BAYESWAVE (Cornish and Littenberg 2015) codes. The O2 results consider only a three-detector (3-det) network; assuming an instrument duty cycle of 70-75\%, this would be operational 34$42 \%$ of the time. The BAYESWAVE results are only for $\mathrm{O} 1$ and include only events that could be detected by the code. The shaded areas indicate the $68 \%$ confidence intervals on the cumulative distributions. A detection threshold of a false alarm rate of approximately $1 \mathrm{yr}^{-1}$ is used for CWB and LIB, and BAYESWAVE is run as a follow-up for CWB triggers. Results are taken from Essick et al. (2015) and Bécsy et al. (2016)

\section{Observing scenarios}

In this section we estimate the sensitivity, possible number of detections, and localization capability for each of the observing runs laid out in Sect. 2.2. We discuss each future observing run in turn and also summarize the results in Table 3.

In the following, we estimate the expected number of BNS coalescence detections using the inferred $90 \%$ credible range for the BNS source rate density, 320$4740 \mathrm{Gpc}^{-3} \mathrm{yr}^{-1}$ (Abbott et al. 2017i). Given the detectors' noise spectral densities, the $\rho_{c}$ detection threshold can be converted into the (source sky-location and orientation averaged) BNS sensitive detection range $R_{\mathrm{BNS}}$ (Abadie et al. 2010b, 2012g). From this, the BNS source rate density can be converted into an estimate of the number of expected detected events; this estimate carries the large error on the source rate 
Table 2 Percentage of time during the first observing run that the LIGO detectors spent in different operating modes as entered by the on-duty operator

\begin{tabular}{llcc}
\hline Detector & & Hanford & Livingston \\
\hline Operating mode & Observing & $64.6 \%$ & $57.4 \%$ \\
& Locking & $17.9 \%$ & $16.1 \%$ \\
& Environmental & $9.7 \%$ & $19.8 \%$ \\
Maintenance & $4.4 \%$ & $4.9 \%$ \\
Commissioning & $2.9 \%$ & $1.6 \%$ \\
& Planned engineering & $0.1 \%$ & $0.0 \%$ \\
& Other & $0.4 \%$ & $0.4 \%$ \\
\hline
\end{tabular}

Since several factors may influence detector operation at any given time, there is a certain subjectivity to the assignments. Maintenance includes a planned 4 -h weekly period ( $\sim 2.4 \%$ of the total). Coincident operation of the detectors occurred $\sim 43 \%$ of the time

density. Similar estimates may be made for NS-BH binaries using the fact that the NS-BH range is approximately a factor of 1.6 larger than the BNS range, ${ }^{8}$ though the uncertainty in the NS-BH source rate density is slightly larger (Abadie et al. 2010b). We assume a nominal $\rho_{c}$ threshold of 12 , at which the expected FAR is $\sim 10^{-2} \mathrm{yr}^{-1}$. However, such a stringent threshold may not be appropriate for selecting candidate triggers for electromagnetic follow-up. For example, selecting CBC candidates at thresholds corresponding to a higher background rate of $1 \mathrm{yr}^{-1}\left(100 \mathrm{yr}^{-1}\right)$ would increase the number of true signals subject to electromagnetic follow-up by about $30 \%(90 \%)$. The area localization for these low-threshold signals is, on average, only fractionally worse than for the high-threshold population-by approximately $20 \%$ $(60 \%)$. The localization of NS-BH signals is expected to be similar to that of BNS signals.

For typical burst sources, the gravitational waveform is not well known. However, the performance of burst searches is largely independent of the detailed waveform morphology (Abadie et al. 2012a; Essick et al. 2015), allowing us to quote an approximate sensitive range determined by the total energy $E_{\mathrm{GW}}$ emitted in GWs, the central frequency $f_{0}$ of the burst, the detector noise spectrum $S(f)$, and the single-detector SNR threshold $\rho_{\text {det }}($ Sutton 2013),

$$
R_{\text {burst }} \simeq\left[\frac{G}{2 \pi^{2} c^{3}} \frac{E_{\mathrm{GW}}}{S\left(f_{0}\right) f_{0}^{2} \rho_{\mathrm{det}}^{2}}\right]^{1 / 2} .
$$

In this article, we quote ranges using $E_{\mathrm{GW}}=10^{-2} M_{\odot} c^{2}$ and $f_{0}=150 \mathrm{~Hz} ; E_{\mathrm{GW}}=$ $10^{-2} M_{\odot} c^{2}$ is an optimistic value for GW emission from stellar collapse (e.g., Abadie et al. 2012e); the uncertainty in $E_{\mathrm{GW}}$ means that the quoted burst ranges are more uncertain than their BNS counterparts. We use a single-detector SNR threshold of 8, corresponding to a typical network SNR of $\sim 12$.

\footnotetext{
8 This assumes a black hole mass of $5 M_{\odot}$.
} 
The run durations discussed below are in calendar time. In O1, the H1-L1 network had a duty factor of approximately $43 \%$. Table 2 illustrates how the up time for each detector was impacted by various activities or the environment. The two biggest nonobserving categories for each detector are Locking and Environmental. Locking refers to the amount of time spent in bringing the interferometers from an uncontrolled state to their lowest noise configuration (Staley et al. 2014). Environmental effects include earthquakes, wind and the microseism noise arising from ocean storms (Effler et al. 2015; Abbott et al. 2016d). The latter two effects have seasonal variation, with the prevalence of storms being higher during the winter months. L1 has a greater sensitivity to microseism noise and to earthquakes than $\mathrm{H} 1$ mainly due to the local geophysical environment (Daw et al. 2004). During O1, L1 lost over twice as much observing time to earthquakes, microseism noise and wind than did H1. While we can expect some improvement in duty factors from operating during non-winter months, we can continue to expect at least a 10\% impact on operating time from environmental effects. Adding in maintenance, both planned and unplanned, and time spent in locking we currently expect duty factors of at most 70-75\% for each instrument during extended runs. Assuming downtime periods are uncorrelated among detectors, this means that all detectors in a three-detector network will be operating in coincidence approximately $34-42 \%$ of the time, and at least two detectors will be operating for $78-84 \%$ of the time. For a four-detector network, three or more detectors will be operational around 65-74\% of the time, and for a five-detector network, three of more detectors will be operating for $84-90 \%$ of the time. Our estimates for the expected number of detections and the fraction of sources localized account for these duty cycles. The downtime periods are sometimes correlated between detectors, for example, planned maintenance periods are often coordinated, and so these coincidence times may be conservative estimates. The number of detections also account for the uncertainty in the detector sensitive ranges as indicated in Fig. 1, but do not include any cosmological evolution of the merger rate.

\section{$4.12015-2016$ run (O1): aLIGO}

This was the first advanced-detector observing run, lasting four months, starting 12 September 2015 and ending 19 January 2016.

The aLIGO sensitivity was expected to be similar to the early band in Fig. 1, with a BNS range of 40-80 Mpc, and a burst range of 40-60 Mpc for $E_{\mathrm{GW}}=10^{-2} M_{\odot} c^{2}$. The achieved sensitivity was at the better end of this span, with a BNS range of 60-80 Mpc.

The O1 BNS search volume was $\sim 2 \times 10^{5} \mathrm{Mpc}^{3} \mathrm{yr}$, and the dominant source of uncertainty on this value is the calibration of the detectors (Abbott et al. 2016q). The search volume is $V_{z} T$, where $V_{z}=(4 \pi / 3) R^{3}$ is the time-averaged volume surveyed and $T$ is the observing time incorporating the effects of the detectors' duty cycles. We would therefore expect 0.05-1 BNS detections. No BNS detections were made, consistent with these expectations (Abbott et al. 2016q).

With the two-detector H1-L1 network any detected events are unlikely to be well localized. A full parameter-estimation study using realistic detector noise and an 
astrophysically-motivated source catalog has been completed for 2015-2016 (Berry et al. 2015). ${ }^{9}$ This used a noise curve in the middle of the early range shown in Fig. 1the early curve specified in Barsotti and Fritschel (2012). The distribution of results is shown in Fig. 6. In Table 3, we present results calculated using BAYESTAR (Singer and Price 2016) for a population of BNS signals, assuming an SNR threshold of 12; the results agree with those of Berry et al. (2015). The median $90 \%$ credible region for is $460-530 \mathrm{deg}^{2}$; the searched area $A_{*}^{\mathrm{BNS}}$ is smaller than $20 \mathrm{deg}^{2}$ for $14-17 \%$ of events and smaller than $5 \mathrm{deg}^{2}$ in $4-6 \%$.

Equivalent (but not directly comparable) results for bursts are found in Essick et al. (2015). Specific results depend upon the waveform morphology used, but the median searched area is 1-2 times larger than for BNS signals; part of this difference is due to the burst study using a less-stringent FAR threshold of $\sim 1 \mathrm{yr}^{-1}$. The distribution of searched areas for four waveform morphologies are shown in Fig. 7.

The localizations of GW150914, GW151226 and LVT151012 exhibit the characteristic broken arc for a two-detector network (Abbott et al. 2016i, c). The $90 \%$ credible regions are $230 \mathrm{deg}^{2}, 850 \mathrm{deg}^{2}$ and $1600 \mathrm{deg}^{2}$ respectively (Abbott et al. 2016c). The sky localization for a CBC signal consistent with the properties of GW150914 is shown in Fig. 8. This shows the localization with the two-detector O1 network as well as with other detector network configurations (Gaebel and Veitch 2017).

The poor localization from a two-detector network makes follow-up challenging. The electromagnetic follow-up effort for GW150914 is described in Abbott et al. (2016i), Abbott et al. (2016n), and the search for coincident neutrinos is described in Adrian-Martinez et al. (2016), Albert et al. (2017c).

\subsection{6-2017 run (O2): aLIGO joined by AdV}

This was an approximately nine-month run with three detectors for the second part of the run. The aLIGO performance was expected to be similar to the mid band in Fig. 1, with a BNS range of $80-120 \mathrm{Mpc}$, and a burst range of $60-75 \mathrm{Mpc}$ for $E_{\mathrm{GW}}=10^{-2} M_{\odot} c^{2}:$ the achieved BNS range is towards the lower part of this band, around 60-100 Mpc (Abbott et al. 2017f). The AdV range was anticipated to be within the early band in Fig. 1, approximately 20-65 Mpc for BNS and 20-40 Mpc for bursts. On 1 August 2017, AdV joined O2 with a BNS range of 25-30 Mpc.

The potential improvement in sky localization from the addition of a third detector is illustrated in Fig. 8.

Anticipated BNS sky localization for 2016-2017 (in addition to 2015-2016) was investigated in Singer et al. (2014). This assumed a noise curve which lies in the middle of the mid range in Fig. 1 for aLIGO - the mid curve specified in Barsotti and Fritschel (2012) — and the geometric mean of the upper and lower bounds of the mid region in Fig. 1 for AdV. The distribution of results is shown in Fig. 6. In Table 3, we give results for an astrophysically-motivated BNS population, with an SNR threshold of 12, assuming a three-detector network with each detector having

\footnotetext{
9 This study used noise from the sixth science run of initial LIGO, recolored to the expected O1 sensitivity curve. The source catalog, as well as the analysis pipeline, is shared with Singer et al. (2014).
} 
Table 3 Summary of a plausible observing schedule, expected sensitivities, and source localization with the Advanced LIGO, Advanced Virgo and KAGRA detectors, which will be strongly dependent on the detectors' commissioning progress

\begin{tabular}{|c|c|c|c|c|c|c|c|}
\hline \multicolumn{3}{|l|}{ Epoch } & $2015-2016$ & $2016-2017$ & $2018-2019$ & $2020+$ & $2024+$ \\
\hline \multicolumn{3}{|c|}{ Planned run duration } & 4 months & 9 months & 12 months & (per year) & (per year) \\
\hline \multirow{3}{*}{\multicolumn{2}{|c|}{ Expected burst range/Mpc }} & LIGO & $40-60$ & $60-75$ & $75-90$ & 105 & 105 \\
\hline & & Virgo & - & $20-40$ & $40-50$ & $40-70$ & 80 \\
\hline & & KAGRA & - & - & - & - & 100 \\
\hline \multirow{3}{*}{\multicolumn{2}{|c|}{ Expected BNS range/Mpc }} & LIGO & $40-80$ & $80-120$ & $120-170$ & 190 & 190 \\
\hline & & Virgo & - & $20-65$ & $65-85$ & $65-115$ & 125 \\
\hline & & KAGRA & - & - & - & - & 140 \\
\hline \multirow{3}{*}{\multicolumn{2}{|c|}{ Achieved BNS range/Mpc }} & LIGO & $60-80$ & $60-100$ & - & - & - \\
\hline & & Virgo & - & $25-30$ & - & - & - \\
\hline & & KAGRA & - & - & - & - & - \\
\hline \multicolumn{3}{|c|}{ Estimated BNS detections } & $0.05-1$ & $0.2-4.5$ & $1-50$ & $4-80$ & $11-180$ \\
\hline \multicolumn{3}{|c|}{ Actual BNS detections } & 0 & 1 & - & - & - \\
\hline \multirow[t]{3}{*}{$90 \% \mathrm{CR}$} & $\%$ within & $5 \mathrm{deg}^{2}$ & $<1$ & $1-5$ & $1-4$ & $3-7$ & $23-30$ \\
\hline & & $20 \mathrm{deg}^{2}$ & $<1$ & $7-14$ & $12-21$ & $14-22$ & $65-73$ \\
\hline & Median/deg ${ }^{2}$ & & $460-530$ & $230-320$ & $120-180$ & $110-180$ & $9-12$ \\
\hline \multirow[t]{2}{*}{ Searched area } & $\%$ within & $5 \mathrm{deg}^{2}$ & $4-6$ & $15-21$ & $20-26$ & $23-29$ & $62-67$ \\
\hline & & $20 \mathrm{deg}^{2}$ & $14-17$ & $33-41$ & $42-50$ & $44-52$ & $87-90$ \\
\hline
\end{tabular}

Ranges reflect the uncertainty in the detector noise spectra shown in Fig. 1. The burst ranges assume standardcandle emission of $10^{-2} M_{\odot} c^{2}$ in gravitational waves at $150 \mathrm{~Hz}$ and scale as $E_{\mathrm{GW}}^{1 / 2}$, so it is greater for more energetic sources (such as binary black holes). The binary neutron star (BNS) localization is characterized by the size of the $90 \%$ credible region (CR) and the searched area. These are calculated by running the BAYESTAR rapid sky-localization code (Singer and Price 2016) on a Monte Carlo sample of simulated signals, assuming senisivity curves in the middle of the plausible ranges (the geometric means of the upper and lower bounds). The variation in the localization reflects both the variation in duty cycle between $70 \%$ and $75 \%$ as well as Monte Carlo statistical uncertainty. The estimated number of BNS detections uses the actual ranges for 2015-2016 and 2017-2018, and the expected range otherwise; future runs assume a $70-75 \%$ duty cycle for each instrument. The BNS detection numbers also account for the uncertainty in the BNS source rate density (Abbott et al. 2017i). Estimated BNS detection numbers and localization estimates are computed assuming a signal-to-noise ratio greater than $\sim 12$. Burst localizations are expected to be broadly similar to those derived from timing triangulation, but vary depending on the signal bandwidth; the median burst searched area (with a false alarm rate of $\sim 1 \mathrm{yr}^{-1}$ ) may be a factor of $\sim 2-3$ larger than the values quoted for BNS signals (Essick et al. 2015). No burst detection numbers are given, since the source rates are currently unknown. Localization numbers for 2016-2017 include Virgo, and do not take into account that Virgo only joined the observations for the latter part the run. The 2024+ scenario includes LIGO-India at design sensitivity

an individual duty cycle of $70-75 \%$. The results are calculated using BAYESTAR. The median $90 \%$ credible region is $230-320 \mathrm{deg}^{2}$, and 7-13\% of events are expected to have $\mathrm{CR}_{0.9}^{\mathrm{BNS}}$ smaller than $20 \mathrm{deg}^{2}$. The searched area is smaller than $20 \mathrm{deg}^{2}$ for 33$41 \%$ of events and smaller than $5 \mathrm{deg}^{2}$ for 16-21\%. The burst study (Essick et al. 2015) 


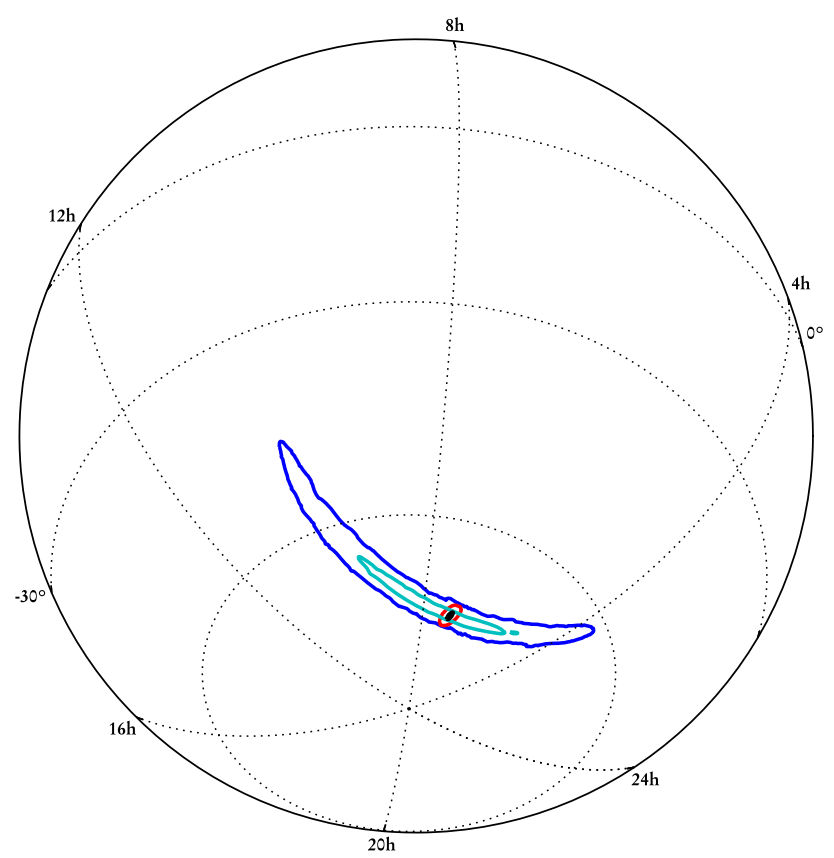

Fig. 8 Sky localization of a signal with parameters consistent with those for GW150914. The lines enclose the $90 \%$ credible regions with different detector networks. Dark blue is for the O1 two-detector network; light blue is for the same Hanford-Livingston network at design sensitivity; red is for the three-detector network including Virgo, with all detectors at early sensitivity, similar to what was expected for O2, and black is for the three detector network at design sensitivity. The plot is an orthographic projection with right ascension measured in hours and declination measured in degrees. Results taken from Gaebel and Veitch (2017)

gives approximately equivalent results, producing median searched areas a factor of 2-3 larger than the BNS results; these results are shown in Fig. 7.

GW170104 (Abbott et al. 2017f) and GW170608 (Abbott et al. 2017g) were detected prior to Virgo joining O2. Therefore, like the O1 events, they have large $90 \%$ credible areas of $1200 \mathrm{deg}^{2}$ and $860 \mathrm{deg}^{2}$ respectively. The addition of Virgo made a significant impact for GW170814 (Abbott et al. 2017h) and GW170817 (Abbott et al. 2017i). For GW170814, the initial BAYESTAR 90\% credible area is reduced from $1160 \mathrm{deg}^{2}$ to $100 \mathrm{deg}^{2}$ with the addition of Virgo, and the final LALINFERENCE threedetector localization is $60 \mathrm{deg}^{2}$. For GW170817, the initial BAYESTAR 90\% credible area is reduced from $190 \mathrm{deg}^{2}$ to $31 \mathrm{deg}^{2}$ with the addition of Virgo, and the final LALINFERENCE three-detector localization is $28 \mathrm{deg}^{2}$. The inclusion of the third detector to the network enhances localization whether or not it detects the signal, provided that it could detect the signal, as the observed amplitude constrains the source position. As a result of being observed with a three-detector network, and its high SNR, GW170817 has the best GW localization to date.

GW170817 is the first GW signal to have a confirmed electromagnetic counterpart. The discoveries associated with this detection are highlighted in Sect. 3. An overview of the extensive multi-messenger observations accompanying GW170817 is given in Abbott et al. (2017k). 


\subsection{8-2019 run (O3): aLIGO 120-170 Mpc, AdV 65-85 Mpc}

This is envisioned to be a year long run with three detectors. The aLIGO and AdV sensitivities will be similar to the late and mid bands of Fig. 1 respectively, with BNS ranges of 120-170 Mpc and 65-85 Mpc, and burst ranges of 75-90 Mpc and 40$50 \mathrm{Mpc}$ for $E_{\mathrm{GW}}=10^{-2} M_{\odot} c^{2}$. This gives an expected update 1-50 BNS detections. Both the range and the typical sky localization should increase relative to the 20162017 run. Table 3 gives BAYESTAR localizations assuming detector sensitivities which are the geometric means of the upper and lower bounds of the relevant bands in Fig. 1. The median $90 \%$ credible region is $120-180 \mathrm{deg}^{2}$, and $12-21 \%$ of events are expected to have $\mathrm{CR}_{0.9}^{\mathrm{BNS}}$ smaller than $20 \mathrm{deg}^{2}$.

\subsection{0+ runs: aLIGO 190 Mpc, AdV 65-125 Mpc}

At this point we anticipate extended runs with the detectors at or near design sensitivity. The aLIGO detectors are expected to have a sensitivity curve similar to the design curve of Fig. 1. AdV may be operating similarly to the late band, eventually reaching the design sensitivity circa 2021. Potential localization for a GW150914-like BBH signal is shown in Fig. 8. The fraction of signals localized to areas of a few square degrees is increased compared to previous runs. This is due to the much larger detector bandwidths, particularly for $\mathrm{AdV}$, as well as the increased sensitivity of the network; see Fig. 1.

\subsection{4+ runs: aLIGO (including LIGO-India) 190 Mpc, AdV 125 Mpc, KAGRA 140 Mpc}

The five-site network incorporating LIGO-India at design sensitivity would have both improved sensitivity and better localization capabilities. The per-year BNS search volume increases giving an expected 11-180 BNS detections annually. The addition of more detector sites leads to good source localization over the whole sky (Schutz 2011; Veitch et al. 2012; Nissanke et al. 2013; Rodriguez et al. 2014). Table 3 gives BAYESTAR localizations for an astrophysical population of BNSs, assuming design sensitivity and a 70-75\% duty cycle for each detector. The median $90 \%$ credible region is $9-12 \mathrm{deg}^{2}, 65-73 \%$ of events are expected to have $\mathrm{CR}_{0.9}^{\mathrm{BNS}}$ smaller than $20 \mathrm{deg}^{2}$, and the searched area is less than $20 \mathrm{deg}^{2}$ for $87-90 \%$.

\section{Conclusions}

We have presented possible observing scenarios for the Advanced LIGO, Advanced Virgo and KAGRA network of GW detectors, with emphasis on the expected sensitivities and sky-localization accuracies. This network began operation in September 2015 with the two LIGO detectors. Virgo joined the network in August 2017, dramatically improving sky localization. With a four- or five-site detector network at design 
sensitivity, we may expect a significant fraction of GW signals to be localized to within a few square degrees by GW observations alone.

The first BBH detection was made promptly after the start of observations in September 2015; they are the most commonly detected GW source, but are not a promising target for multi-messenger observations. GW detections will become more common as the sensitivity of the network improves. The first BNS coalescence was detected in August 2017. This was accompanied by observations across the electromagnetic spectrum (Abbott et al. 2017k). Multi-messenger observations of BNSs provide new insights into binary evolution, nuclear physics, cosmology and gravitational physics.

Optimizing the multi-messenger follow-up and source identification is an outstanding research topic (e.g., Abadie et al. 2012b; Aasi et al. 2014a; Kasliwal and Nissanke 2014; Singer et al. 2014; Cannon et al. 2012; Evans et al. 2016a; Gehrels et al. 2016; Ghosh et al. 2016; Chan et al. 2017; Rana et al. 2017; Salafia et al. 2017; Patricelli et al. 2018). Triggering of focused searches in GW data by electromagnetically-detected events can also help in recovering otherwise hidden GW signals (Aasi et al. 2014c). Multi-messenger follow-up of GW candidates may help confirm GW candidates that would not be confidently identified from GW observations alone. However, such follow-ups need to deal with large position uncertainties, with areas of many tens to thousands of square degrees. This is likely to remain the situation until late in the decade.

The purpose of this article is to provide information to the astronomy community to facilitate planning for multi-messenger astronomy with advanced GW detectors. While the scenarios described here are our best current projections, they will evolve as detector installation and commissioning progress. We will therefore update this article regularly.

Acknowledgements The authors gratefully acknowledge the support of the United States National Science Foundation (NSF) for the construction and operation of the LIGO Laboratory and Advanced LIGO as well as the Science and Technology Facilities Council (STFC) of the United Kingdom, the Max-Planck-Society (MPS), and the State of Niedersachsen/Germany for support of the construction of Advanced LIGO and construction and operation of the GEO 600 detector. Additional support for Advanced LIGO was provided by the Australian Research Council. The authors gratefully acknowledge the Italian Istituto Nazionale di Fisica Nucleare (INFN), the French Centre National de la Recherche Scientifique (CNRS) and the Foundation for Fundamental Research on Matter supported by the Netherlands Organisation for Scientific Research, for the construction and operation of the Virgo detector and the creation and support of the EGO consortium. The authors also gratefully acknowledge research support from these agencies as well as by the Council of Scientific and Industrial Research of India, the Department of Science and Technology, India, the Science \& Engineering Research Board (SERB), India, the Ministry of Human Resource Development, India, the Spanish Agencia Estatal de Investigación, the Vicepresidència i Conselleria d'Innovació, Recerca i Turisme and the Conselleria d'Educació i Universitat del Govern de les Illes Balears, the Conselleria d'Educació, Investigació, Cultura i Esport de la Generalitat Valenciana, the National Science Centre of Poland, the Swiss National Science Foundation (SNSF), the Russian Foundation for Basic Research, the Russian Science Foundation, the European Commission, the European Regional Development Funds (ERDF), the Royal Society, the Scottish Funding Council, the Scottish Universities Physics Alliance, the Hungarian Scientific Research Fund (OTKA), the Lyon Institute of Origins (LIO), the Paris Île-de-France Region, the National Research, Development and Innovation Office Hungary (NKFI), the National Research Foundation of Korea, Industry Canada and the Province of Ontario through the Ministry of Economic Development and Innovation, the Natural Science and Engineering Research Council Canada, the Canadian Institute for Advanced Research, the Brazilian Ministry of Science, Technology, Innovations, and Communications, the 
International Center for Theoretical Physics South American Institute for Fundamental Research (ICTPSAIFR), the Research Grants Council of Hong Kong, the National Natural Science Foundation of China (NSFC), the Leverhulme Trust, the Research Corporation, the Ministry of Science and Technology (MOST), Taiwan and the Kavli Foundation. The authors gratefully acknowledge the support of the NSF, STFC, MPS, INFN, CNRS and the State of Niedersachsen/Germany for provision of computational resources. The authors gratefully acknowledge the support in Japan by MEXT, JSPS Leading-edge Research Infrastructure Program, JSPS Grant-in-Aid for Specially Promoted Research 26000005, MEXT Grant-in-Aid for Scientific Research on Innovative Areas 24103005, JSPS Core-to-Core Program, A. Advanced Research Networks, the joint research program of the Institute for Cosmic Ray Research, University of Tokyo, and Computing Infrastructure Project of KISTI-GSDC in Korea. This article has been assigned LIGO Document number P1200087, Virgo Document number VIR-0288A-12, and KAGRA Document number JGW-P1706792.

Open Access This article is distributed under the terms of the Creative Commons Attribution 4.0 International License (http://creativecommons.org/licenses/by/4.0/), which permits unrestricted use, distribution, and reproduction in any medium, provided you give appropriate credit to the original author(s) and the source, provide a link to the Creative Commons license, and indicate if changes were made.

\section{A Changes between versions}

Since publication of the previous version (Aasi et al. 2016), several updates to the document have been made. The most significant changes are the inclusion of details regarding KAGRA, and results from O1 and O2, including GW170817, the first detection with a unambiguous multi-messenger counterpart (Abbott et al. 2017k). The key differences are outlined below.

\section{A.1 Updates to detector commissioning}

The plausible detector scenarios remain largely unchanged, but details of $\mathrm{O} 1$ and $\mathrm{O} 2$ have been updated. Specific updates to the detector scenarios are:

1. The addition of KAGRA to Figs. 1 and 2.

2. Table 1 has been added which includes BNS ranges and $30 M_{\odot}+30 M_{\odot} \mathrm{BBH}$ ranges.

3. The $\mathrm{O} 1$ sensitivity has been updated following the completion of the run (12 September 2015-19 January 2016). We believed that the O1 BNS range would plausibly be $40-80 \mathrm{Mpc}$, and the actual range was $60-80 \mathrm{Mpc}$.

4. The formal transition to $\mathrm{O} 2$ was 30 November 2016, and the run ended 25 August 2017. The run is approximately nine calendar months in duration, but includes a two-week break at the end of December and a three-week commissioning break in May. The achieved aLIGO BNS range was approximately 60-100 Mpc (Abbott et al. 2017f).

5. The AdV detector officially joined the $\mathrm{O} 2$ run on 1 August 2017. The achieved AdV BNS range was approximately $25-30 \mathrm{Mpc}$.

6. As a consequence of the extended $\mathrm{O} 2$ run, the start of $\mathrm{O} 3$ is now expected to be in 2018, approximately a year after the end of $\mathrm{O} 2$. $\mathrm{O} 3$ is now planned to be a year in duration.

7. Figure 2 has been updated to show the current planned timeline. 
8. Based on our experience in $\mathrm{O} 1$ and $\mathrm{O} 2$, we have revised our predicted duty cycles, we believe that single-detector duty cycles of 70-75\% for extended runs are more realistic than the previous value of $80 \%$.

9. We have updated the final observing scenario, the 2024+ case, to be a five-detector network including KAGRA.

10. Progress has been made towards establishing LIGO-India, with the the Indian government granting in-principle approval; however, it is still too early to give a definite timeline for the observing schedule. 2024 is the earliest we imagine it could be operational.

11. Some of the BNS ranges associated with different observing scenarios have changed as a result of using an updated calculation, including cosmological corrections. For all cosmological calculations, we assume a flat cosmology with Hubble parameter $H_{0}=67.9 \mathrm{~km} \mathrm{~s}^{-1} \mathrm{Mpc}^{-1}$, and density parameters $\Omega_{\mathrm{m}}=0.3065$ and $\Omega_{\Lambda}=0.6935$ (Ade et al. 2016). The sensitivity curves themselves have not been modified. Ranges are rounded to the nearest megaparsec below $15 \mathrm{Mpc}$, rounded to the nearest $5 \mathrm{Mpc}$ between $15 \mathrm{Mpc}$ and $1000 \mathrm{Mpc}$, and to the nearest $10 \mathrm{Mpc}$ above $1000 \mathrm{Mpc}$.

\section{A.2 Updates to data analysis}

In addition to the progress made with regards to the detectors, there have also been significant advances from analysing the data. We now include results from O1 (Abbott et al. 2016c, q, 2017b) and initial results from O2; these include the first detections of BBH and BNS systems (Abbott et al. 2016k, g, c, 2017f, g, h,i). Multi-messenger searches for counterparts to these detections are described in Abbott et al. (2016i, n); Adrian-Martinez et al. (2016); Albert et al. (2017c); Abbott et al. (2017k). Specific updates that have been made are:

1. Figure 3 has been updated to include $\mathrm{O} 1$ results; the surrounding text has been updated to discuss the detection pipelines used in O1 (Abbott et al. 2016e, c, l, 2017b).

2. Results using the BAYESWAVE algorithm (Bécsy et al. 2016), appropriate for O1, have been included in Fig. 7.

3. Figure 8 shows a sky localization, created using current parameter-estimation techniques, illustrating how localization improves for different network configurations for a GW150914-like signal (Gaebel and Veitch 2017).

4. Table 3 now includes numbers summarising results from $\mathrm{O} 1$ and the new 2024+ scenario including KAGRA. Sky localization numbers for are now calculated using BAYESTAR (Singer and Price 2016) for all epochs.

5. BNS merger rates have been updated following the observation of GW170817. The post-detection range is $320-4740 \mathrm{Gpc}^{-3} \mathrm{yr}^{-1}$ (Abbott et al. 2017i), consistent with the previous expectation of $10-10^{4} \mathrm{Gpc}^{-3} \mathrm{yr}^{-1}$ (Abadie et al. 2010b).

We defer describing the full results of $\mathrm{O} 2$ until a future update. 


\section{References}

Aab A et al (2016) Ultrahigh-energy neutrino follow-up of gravitational wave events GW150914 and GW151226 with the Pierre Auger Observatory. Phys Rev D 94:122007. https://doi.org/10.1103/ PhysRevD.94.122007. arXiv:1608.07378

Aasi J et al (2012) The characterization of Virgo data and its impact on gravitational-wave searches. Class Quantum Grav 29:155002. https://doi.org/10.1088/0264-9381/29/15/155002. arXiv:1203.5613

Aasi J et al (2013a) Enhancing the sensitivity of the LIGO gravitational wave detector by using squeezed states of light. Nat Photon 7:613-619. https://doi.org/10.1038/nphoton.2013.177. arXiv:1310.0383

Aasi J et al (2013b) Open call for partnership for the EM identification and follow-up of GW candidate events. Technical report LIGO M1300550-v3 / VIR-0494E-13, LIGO, Pasadena, CA. https://dcc.ligo. org/LIGO-M1300550-v8/public

Aasi J et al (2013c) Parameter estimation for compact binary coalescence signals with the first generation gravitational-wave detector network. Phys Rev D 88:062001. https://doi.org/10.1103/PhysRevD.88. 062001. arXiv:1304.1775

Aasi J et al (2014a) First searches for optical counterparts to gravitational-wave candidate events. Astrophys J Suppl 211:7. https://doi.org/10.1088/0067-0049/211/1/7. arXiv:1310.2314

Aasi J et al (2014b) Methods and results of a search for gravitational waves associated with gamma-ray bursts using the GEO600, LIGO, and Virgo detectors. Phys Rev D 89:122004. https://doi.org/10.1103/ PhysRevD.89.122004. arXiv: 1405.1053

Aasi J et al (2014c) Search for gravitational waves associated with $\gamma$-ray bursts detected by the Interplanetary Network. Phys Rev Lett 113:011102. https://doi.org/10.1103/PhysRevLett.113.011102. arXiv: 1403.6639

Aasi J et al (2015a) Advanced LIGO. Class Quantum Grav 32:074001. https://doi.org/10.1088/0264-9381/ 32/7/074001. arXiv:1411.4547

Aasi J et al (2015b) Characterization of the LIGO detectors during their sixth science run. Class Quantum Grav 32:115012. https://doi.org/10.1088/0264-9381/32/11/115012. arXiv:1410.7764

Aasi J et al (2015c) Instrument science white paper. Technical report LIGO-T1400316-v4, LIGO, Pasadena, CA. https://dcc.ligo.org/LIGO-T1400316/public

Aasi J et al (2016) Prospects for observing and localizing gravitational-wave transients with Advanced LIGO and Advanced Virgo. Living Rev Relativ 19:1. https://doi.org/10.1007//rr-2016-1. arXiv:1304.0670v3

Abadie J et al (2010a) All-sky search for gravitational-wave bursts in the first joint LIGO-GEO-Virgo run. Phys Rev D 81:102001. https://doi.org/10.1103/PhysRevD.85.089905. arXiv:1002.1036

Abadie J et al (2010b) Predictions for the rates of compact binary coalescences observable by ground-based gravitational-wave detectors. Class Quantum Grav 27:173001. https://doi.org/10.1088/0264-9381/ 27/17/173001. arXiv:1003.2480

Abadie J et al (2011) A gravitational wave observatory operating beyond the quantum shot-noise limit: squeezed light in application. Nat Phys 7:962-965. https://doi.org/10.1038/nphys2083. arXiv: 1109.2295

Abadie J et al (2012a) All-sky search for gravitational-wave bursts in the second joint LIGO-Virgo run. Phys Rev D 85:122007. https://doi.org/10.1103/PhysRevD.85.122007. arXiv:1202.2788

Abadie J et al (2012b) First low-latency LIGO+Virgo search for binary inspirals and their electromagnetic counterparts. Astron Astrophys 541:A155. https://doi.org/10.1051/0004-6361/201218860. arXiv: 1112.6005

Abadie J et al (2012c) Implementation and testing of the first prompt search for gravitational wave transients with electromagnetic counterparts. Astron Astrophys 539:A124. https://doi.org/10.1051/0004-6361/ 201118219. arXiv:1109.3498

Abadie J et al (2012d) LSC and Virgo policy on releasing gravitational wave triggers to the public in the advanced detectors era. Technical report LIGO M1200055-v2 / VIR-0173A-12, LIGO, Pasadena, CA. https://dcc.ligo.org/LIGO-M1200055-v2/public

Abadie J et al (2012e) Search for gravitational waves associated with gamma-ray bursts during LIGO science run 6 and Virgo science runs 2 and 3. Astrophys J 760:12. https://doi.org/10.1088/0004-637X/760/1/ 12. arXiv: 1205.2216

Abadie J et al (2012f) Search for gravitational waves from low mass compact binary coalescence in LIGO's sixth science run and Virgo's science runs 2 and 3. Phys Rev D 85:082002. https://doi.org/10.1103/ PhysRevD.85.082002. arXiv:1111.7314 
Abadie J et al (2012g) Sensitivity achieved by the LIGO and Virgo gravitational wave detectors during LIGO's sixth and Virgo's second and third science runs. ArXiv e-prints arXiv:1203.2674

Abbott BP et al (2016a) All-sky search for long-duration gravitational wave transients with initial LIGO. Phys Rev D 93:042005. https://doi.org/10.1103/PhysRevD.93.042005. arXiv:1511.04398

Abbott BP et al (2016b) Astrophysical implications of the binary black-hole merger GW150914. Astrophys J Lett 818:L22. https://doi.org/10.3847/2041-8205/818/2/L22. arXiv:1602.03846

Abbott BP et al (2016c) Binary black hole mergers in the first Advanced LIGO observing run. Phys Rev X 6:041015. https://doi.org/10.1103/PhysRevX.6.041015. arXiv:1606.04856

Abbott BP et al (2016d) Characterization of transient noise in Advanced LIGO relevant to gravitational wave signal GW150914. Class Quantum Grav 33:134001. https://doi.org/10.1088/0264-9381/33/13/ 134001. arXiv:1602.03844

Abbott BP et al (2016e) GW150914: First results from the search for binary black hole coalescence with Advanced LIGO. Phys Rev D 93:122003. https://doi.org/10.1103/PhysRevD.93.122003. arXiv: 1602.03839

Abbott BP et al (2016f) GW150914: The Advanced LIGO detectors in the era of first discoveries. Phys Rev Lett 116:131103. https://doi.org/10.1103/PhysRevLett.116.131103. arXiv:1602.03838

Abbott BP et al (2016g) GW151226: Observation of gravitational waves from a 22-solar-mass binary black hole coalescence. Phys Rev Lett 116:241103. https://doi.org/10.1103/PhysRevLett.116.241103. arXiv: 1606.04855

Abbott BP et al (2016h) Improved analysis of GW150914 using a fully spin-precessing waveform model. Phys Rev X 6:041014. https://doi.org/10.1103/PhysRevX.6.041014. arXiv:1606.01210

Abbott BP et al (2016i) Localization and broadband follow-up of the gravitational-wave transient GW150914. Astrophys J Lett 826:L13. https://doi.org/10.3847/2041-8205/826/1/L13. arXiv: 1602.08492

Abbott BP et al (2016j) The LSC-Virgo white paper on gravitational wave searches and astrophysics (2016-2017 edition). Technical report LIGO-T1600115-v6, LIGO, Pasadena, CA. https://dcc.ligo. org/LIGO-T1600115/public

Abbott BP et al (2016k) Observation of gravitational waves from a binary black hole merger. Phys Rev Lett 116:061102. https://doi.org/10.1103/PhysRevLett.116.061102. arXiv:1602.03837

Abbott BP et al (20161) Observing gravitational-wave transient GW150914 with minimal assumptions. Phys Rev D 93:122004. https://doi.org/10.1103/PhysRevD.93.122004. arXiv:1602.03843

Abbott BP et al (2016m) Properties of the binary black hole merger GW150914. Phys Rev Lett 116:241102. https://doi.org/10.1103/PhysRevLett.116.241102. arXiv:1602.03840

Abbott BP et al (2016n) Supplement: localization and broadband follow-up of the gravitationalwave transient GW150914. Astrophys J Suppl 225:8. https://doi.org/10.3847/0067-0049/225/1/8. arXiv: 1604.07864

Abbott BP et al (2016o) Supplement: the rate of binary black hole mergers inferred from Advanced LIGO observations surrounding GW150914. Astrophys J Suppl 227:14. https://doi.org/10.3847/0067-0049/ 227/2/14. arXiv:1606.03939

Abbott BP et al (2016p) The rate of binary black hole mergers inferred from Advanced LIGO observations surrounding GW150914. Astrophys J Lett 833:1. https://doi.org/10.3847/2041-8205/833/1/L1. arXiv: 1602.03842

Abbott BP et al (2016q) Upper limits on the rates of binary neutron star and neutron-star-black-hole mergers from Advanced LIGO's first observing run. Astrophys J Lett 832:L21. https://doi.org/10.3847/20418205/832/2/L21. arXiv:1607.07456

Abbott BP et al (2017a) A gravitational-wave standard siren measurement of the Hubble constant. Nature 551(7678):85-88. https://doi.org/10.1038/nature24471. arXiv:1710.05835

Abbott BP et al (2017b) All-sky search for short gravitational-wave bursts in the first Advanced LIGO run. Phys Rev D 95:042003. https://doi.org/10.1103/PhysRevD.95.042003. arXiv:1611.02972

Abbott BP et al (2017c) Calibration of the Advanced LIGO detectors for the discovery of the binary blackhole merger GW150914. Phys Rev D 95:062003. https://doi.org/10.1103/PhysRevD.95.062003. arXiv: 1602.03845

Abbott BP et al (2017d) Exploring the sensitivity of next generation gravitational wave detectors. Class Quantum Grav 34:044001. https://doi.org/10.1088/1361-6382/aa51f4. arXiv:1607.08697

Abbott BP et al (2017e) Gravitational waves and gamma-rays from a binary neutron star merger: GW170817 and GRB 170817A. Astrophys J Lett 848:L13. https://doi.org/10.3847/2041-8213/ aa920c. arXiv: 1710.05834 
Abbott BP et al (2017f) GW170104: Observation of a 50-solar-mass binary black hole coalescence at redshift 0.2. Phys Rev Lett 118:221101. https://doi.org/10.1103/PhysRevLett.118.221101. arXiv:1706.01812

Abbott BP et al (2017g) GW170608: Observation of a 19 solar-mass binary black hole coalescence. Astrophys J Lett 851:35. https://doi.org/10.3847/2041-8213/aa9f0c. arXiv:1711.05578

Abbott BP et al (2017h) GW170814: A three-detector observation of gravitational waves from a binary black hole coalescence. Phys Rev Lett 119:141101. https://doi.org/10.1103/PhysRevLett.119.141101. arXiv: 1709.09660

Abbott BP et al (2017i) GW170817: Observation of gravitational waves from a binary neutron star inspiral. Phys Rev Lett 119:161101. https://doi.org/10.1103/PhysRevLett.119.161101. arXiv:1710.05832

Abbott BP et al (2017j) The LSC-Virgo white paper on gravitational wave searches and astrophysics (2017-2018 edition). Technical report LIGO-T1700214-v4, LIGO, Pasadena, CA. https://dcc.ligo. org/LIGO-T1700214/public

Abbott BP et al (2017k) Multi-messenger observations of a binary neutron star merger. Astrophys J Lett 848:L12. https://doi.org/10.3847/2041-8213/aa91c9. arXiv:1710.05833

Abbott BP et al (20171) Search for gravitational waves associated with gamma-ray bursts during the first Advanced LIGO observing run and implications for the origin of GRB 150906B. Astrophys J 841:89. https://doi.org/10.3847/1538-4357/aa6c47. arXiv:1611.07947

Abbott BP et al $(2017 \mathrm{~m})$ Search for intermediate mass black hole binaries in the first observing run of Advanced LIGO. Phys Rev D 96:022001. https://doi.org/10.1103/PhysRevD.96.022001. arXiv: 1704.04628

Abbott BP et al (2018) Effects of data quality vetoes on a search for compact binary coalescences in Advanced LIGO's first observing run. Class Quant Grav 35(6):065010. https://doi.org/10.1088/13616382/aaaafa. arXiv: 1710.02185

Abdalla $\mathrm{H}$ et al (2017) TeV gamma-ray observations of the binary neutron star merger GW170817 with H.E.S.S. Astrophys J Lett 850:L22. https://doi.org/10.3847/2041-8213/aa97d2. arXiv:1710.05862

Abe K et al (2016) Search for Neutrinos in Super-Kamiokande associated with Gravitational Wave Events GW150914 and GW151226. Astrophys J Lett 830:L11. https://doi.org/10.3847/2041-8205/830/1/ L11. arXiv:1608.08745

Accadia T et al (2012) Advanced Virgo technical design report. Technical report VIR-0128A-12, Virgo, Cascina. https://tds.ego-gw.it/ql/?c=8940

Acernese F et al (2009) Advanced Virgo baseline design. Technical report VIR-027A-09, Virgo, Cascina. https://tds.ego-gw.it/ql/?c=6589

Acernese F et al (2015) Advanced Virgo: a second-generation interferometric gravitational wave detector. Class Quantum Grav 32:024001. https://doi.org/10.1088/0264-9381/32/2/024001. arXiv:1408.3978

Ackermann M et al (2016) Fermi-LAT observations of the LIGO event GW150914. Astrophys J Lett 823:L2. https://doi.org/10.3847/2041-8205/823/1/L2. arXiv:1602.04488

Adams TS, Meacher D, Clark J, Sutton PJ, Jones G, Minot A (2013) Gravitational-wave detection using multivariate analysis. Phys Rev D 88:062006. https://doi.org/10.1103/PhysRevD.88.062006. arXiv: 1305.5714

Ade PAR et al (2016) Planck 2015 results. XIII. Cosmological parameters. Astron Astrophys 594:A13. https://doi.org/10.1051/0004-6361/201525830. arXiv:1502.01589

Adrian-Martinez S et al (2016) High-energy neutrino follow-up search of gravitational wave event GW150914 with ANTARES and IceCube. Phys Rev D 93:122010. https://doi.org/10.1103/PhysRevD. 93.122010. arXiv:1602.05411

Adriani O et al (2016) CALET Upper Limits on X-ray and Gamma-ray Counterparts of GW151226. Astrophys J Lett 829:L20. https://doi.org/10.3847/2041-8205/829/1/L20. arXiv:1607.00233

Affeldt C et al (2014) Advanced techniques in GEO 600. Class Quantum Grav 31:224002. https://doi.org/ $10.1088 / 0264-9381 / 31 / 22 / 224002$

Agostini M et al (2017) A search for low-energy neutrinos correlated with gravitational wave events GW150914, GW151226 and GW170104 with the Borexino detector. Astrophys J 850:21. https:// doi.org/10.3847/1538-4357/aa9521. arXiv:1706.10176

Ajith P, Fotopoulos N, Privitera S, Neunzert A, Weinstein AJ (2014) Effectual template bank for the detection of gravitational waves from inspiralling compact binaries with generic spins. Phys Rev D 89:084041. https://doi.org/10.1103/PhysRevD.89.084041. arXiv:1210.6666

Akutsu T et al (2018) Construction of KAGRA: an underground gravitational wave observatory. PTEP 2018(1):013F01. https://doi.org/10.1093/ptep/ptx180. arXiv:1712.00148 
Albert A et al (2017a) All-sky search for high-energy neutrinos from gravitational wave event GW170104 with the ANTARES neutrino telescope. Eur Phys J C 77:911. https://doi.org/10.1140/epjc/s10052017-5451-z. arXiv:1710.03020

Albert A et al (2017b) Search for high-energy neutrinos from binary neutron star merger GW170817 with ANTARES, IceCube, and the Pierre Auger Observatory. Astrophys J Lett 850:L35. https://doi.org/ 10.3847/2041-8213/aa9aed. arXiv:1710.05839

Albert A et al (2017c) Search for high-energy neutrinos from gravitational wave event GW151226 and candidate LVT151012 with ANTARES and IceCube. Phys Rev D 96:022005. https://doi.org/10.1103/ PhysRevD.96.022005. arXiv:1703.06298

Alexander KD et al (2017) The electromagnetic counterpart of the binary neutron star merger LIGO/VIRGO GW170817. VI. Radio constraints on a relativistic jet and predictions for late-time emission from the kilonova ejecta. Astrophys J Lett 848:L21. https://doi.org/10.3847/2041-8213/aa905d. arXiv: 1710.05457

Allen B (2005) A $\chi^{2}$ time-frequency discriminator for gravitational wave detection. Phys Rev D 71:062001. https://doi.org/10.1103/PhysRevD.71.062001. arXiv:gr-qc/0405045

Amaro-Seoane P et al (2012) Low-frequency gravitational-wave science with eLISA/NGO. Class Quantum Grav 29:124016. https://doi.org/10.1088/0264-9381/29/12/124016. arXiv:1202.0839

Amaro-Seoane P et al (2013) eLISA/NGO: Astrophysics and cosmology in the gravitational-wave millihertz regime. GW Notes 6:4-110. arXiv:1201.3621

Annis J et al (2016) A dark energy camera search for missing supergiants in the LMC after the Advanced LIGO gravitational-wave event GW150914. Astrophys J Lett 823:L34. https://doi.org/10.3847/20418205/823/2/L34. arXiv:1602.04199

Arai S, Nishizawa A (2017) Generalized framework for testing gravity with gravitational-wave propagation. II. Constraints on Horndeski theory. ArXiv e-prints arXiv:1711.03776

Arcavi I et al (2017a) Optical emission from a kilonova following a gravitational-wave-detected neutron-star merger. Nature 551:64. https://doi.org/10.1038/nature24291. arXiv:1710.05843

Arcavi I et al (2017b) Optical follow-up of gravitational-wave events with Las Cumbres observatory. Astrophys J Lett 848:L33. https://doi.org/10.3847/2041-8213/aa910f. arXiv:1710.05842

Aso Y et al (2013) Interferometer design of the KAGRA gravitational wave detector. Phys Rev D 88:043007. https://doi.org/10.1103/PhysRevD.88.043007. arXiv:1306.6747

Babak S, Balasubramanian R, Churches D, Cokelaer T, Sathyaprakash BS (2006) A template bank to search for gravitational waves from inspiralling compact binaries: I. Physical models. Class Quantum Grav 23:5477-5504. https://doi.org/10.1088/0264-9381/23/18/002. arXiv:gr-qc/0604037

Babak S et al (2013) Searching for gravitational waves from binary coalescence. Phys Rev D 87:024033. https://doi.org/10.1103/PhysRevD.87.024033. arXiv:1208.3491

Bagoly Z et al (2016) Searching for electromagnetic counterpart of LIGO gravitational waves in the Fermi GBM data with ADWO. Astron Astrophys 593:L10. https://doi.org/10.1051/0004-6361/201628569. arXiv: 1603.06611

Baker T, Bellini E, Ferreira PG, Lagos M, Noller J, Sawicki I (2017) Strong constraints on cosmological gravity from GW170817 and GRB 170817A. Phys Rev Lett 119:251301. https://doi.org/10.1103/ PhysRevLett.119.251301. arXiv:1710.06394

Barausse E, Yunes N, Chamberlain K (2016) Theory-agnostic constraints on black-hole dipole radiation with multiband gravitational-wave astrophysics. Phys Rev Lett 116:241104. https://doi.org/10.1103/ PhysRevLett.116.241104. arXiv:1603.04075

Barsotti L, Fritschel P (2012) Early aLIGO configurations: example scenarios toward design sensitivity. Technical report LIGO-T1200307-v4, LIGO, Pasadena, CA. https://dcc.ligo.org/LIGO-T1200307/ public

Bartos I, Kocsis B, Haiman Z, Márka S (2017) Rapid and bright stellar-mass binary black hole mergers in active galactic nuclei. Astrophys J 835:165. https://doi.org/10.3847/1538-4357/835/2/165. arXiv: 1602.03831

Bécsy B, Raffai P, Cornish NJ, Essick R, Kanner J, Katsavounidis E, Littenberg TB, Millhouse M, Vitale S (2016) Parameter estimation for gravitational-wave bursts with the BayesWave pipeline. Astrophys J 839:1. https://doi.org/10.3847/1538-4357/aa63ef. arXiv:1612.02003

Belczynski K et al (2017) The origin of the first neutron star-neutron star merger. ArXiv e-prints arXiv:1712.00632 
Berry CPL et al (2015) Parameter estimation for binary neutron-star coalescences with realistic noise during the Advanced LIGO era. Astrophys J 804:114. https://doi.org/10.1088/0004-637X/804/2/114. arXiv: 1411.6934

Bhalerao V et al (2017) A tale of two transients: GW170104 and GRB170105A. Astrophys J 845:152. https://doi.org/10.3847/1538-4357/aa81d2. arXiv:1706.00024

Blackburn L, Briggs MS, Camp J, Christensen N, Connaughton V, Jenke P, Remillard RA, Veitch J (2015) High-energy electromagnetic offline follow-up of LIGO-Virgo gravitational-wave binary coalescence candidate events. Astrophys J Suppl 217:8. https://doi.org/10.1088/0067-0049/217/1/8. arXiv: 1410.0929

Blanchet L (2014) Gravitational radiation from post-Newtonian sources and inspiralling compact binaries. Living Rev Relativ 17:2. https://doi.org/10.12942/lrr-2014-2. arXiv:1310.1528

Boran S, Desai S, Kahya EO, Woodard RP (2018) GW170817 falsifies dark matter emulators. Phys Rev D 97(4):041501. https://doi.org/10.1103/PhysRevD.97.041501. arXiv:1710.06168

Breivik K et al (2016) Distinguishing between formation channels for binary black holes with LISA. Astrophys J Lett 830:L18. https://doi.org/10.3847/2041-8205/830/1/L18. arXiv:1606.09558

Brocato E et al (2017) GRAWITA: VLT Survey Telescope observations of the gravitational wave sources GW150914 and GW151226. ArXiv e-prints arXiv:1710.05915

Brown DA, Harry I, Lundgren A, Nitz AH (2012) Detecting binary neutron star systems with spin in advanced gravitational-wave detectors. Phys Rev D 86:084017. https://doi.org/10.1103/PhysRevD. 86.084017. arXiv:1207.6406

Brown DD, Miao H, Collins C, Mow-Lowry C, Töyra D, Freise A (2017) Broadband sensitivity enhancement of detuned dual-recycled Michelson interferometers with EPR entanglement. Phys Rev D 96:062003. https://doi.org/10.1103/PhysRevD.96.062003. arXiv:1704.07173

Buonanno A, Iyer B, Ochsner E, Pan Y, Sathyaprakash BS (2009) Comparison of post-Newtonian templates for compact binary inspiral signals in gravitational-wave detectors. Phys Rev D 80:084043. https:// doi.org/10.1103/PhysRevD.80.084043. arXiv:0907.0700

Bustillo JC, Laguna P, Shoemaker D (2017) Detectability of gravitational waves from binary black holes: Impact of precession and higher modes. Phys Rev D 95:104038. https://doi.org/10.1103/PhysRevD. 95.104038. arXiv: 1612.02340

Canizares P, Field SE, Gair JR, Tiglio M (2013) Gravitational wave parameter estimation with compressed likelihood evaluations. Phys Rev D 87:124005. https://doi.org/10.1103/PhysRevD.87.124005. arXiv:1304.0462

Canizares P, Field SE, Gair J, Raymond V, Smith R, Tiglio M (2015) Accelerated gravitational-wave parameter estimation with reduced order modeling. Phys Rev Lett 114:071104. https://doi.org/10. 1103/PhysRevLett.114.071104. arXiv:1404.6284

Cannon K, Cariou R, Chapman A, Crispin-Ortuzar M, Fotopoulos N et al (2012) Toward early-warning detection of gravitational waves from compact binary coalescence. Astrophys J 748:136. https://doi. org/10.1088/0004-637X/748/2/136. arXiv:1107.2665

Cannon K, Hanna C, Peoples J (2015) Likelihood-ratio ranking statistic for compact binary coalescence candidates with rate estimation. ArXiv e-prints arXiv:1504.04632

Capano C, Harry I, Privitera S, Buonanno A (2016) Implementing a search for gravitational waves from binary black holes with nonprecessing spin. Phys Rev D 93:124007. https://doi.org/10.1103/ PhysRevD.93.124007. arXiv:1602.03509

Capano C, Dent T, Hanna C, Hendry M, Hu YM, Messenger C, Veitch J (2017) Systematic errors in estimation of gravitational-wave candidate significance. Phys Rev D 96:082002. https://doi.org/10. 1103/PhysRevD.96.082002. arXiv:1708.06710

Centrella J et al (2010) Black-hole binaries, gravitational waves, and numerical relativity. Rev Mod Phys 82:3069. https://doi.org/10.1103/RevModPhys.82.3069. arXiv:1010.5260

Chan ML, Hu YM, Messenger C, Hendry M, Heng IS (2017) Maximising the detection probability of kilonovae associated with gravitational wave observations. Astrophys J 834:84. https://doi.org/10. 3847/1538-4357/834/1/84. arXiv:1506.04035

Chassande-Mottin E, Miele M, Mohapatra S, Cadonati L (2010) Detection of gravitational-wave bursts with chirplet-like template families. Class Quantum Grav 27:194017. https://doi.org/10.1088/02649381/27/19/194017. arXiv:1005.2876

Chatterji S, Lazzarini A, Stein L, Sutton P, Searle A, Tinto M (2006) Coherent network analysis technique for discriminating gravitational-wave bursts from instrumental noise. Phys Rev D 74:082005. https:// doi.org/10.1103/PhysRevD.74.082005. arXiv:gr-qc/0605002 
Chen HY, Holz DE (2015) Facilitating follow-up of LIGO-Virgo events using rapid sky localization. ArXiv e-prints arXiv:1509.00055

Chen HY, Holz DE, Miller J, Evans M, Vitale S, Creighton J (2017) Distance measures in gravitational-wave astrophysics and cosmology. ArXiv e-prints arXiv:1709.08079

Chornock R et al (2017) The electromagnetic counterpart of the binary neutron star merger LIGO/VIRGO GW170817. IV. Detection of near-infrared signatures of r-process nucleosynthesis with Gemini-south. Astrophys J Lett 848:L19. https://doi.org/10.3847/2041-8213/aa905c. arXiv:1710.05454

Cokelaer T (2007) Gravitational waves from inspiralling compact binaries: hexagonal template placement and its efficiency in detecting physical signals. Phys Rev D 76:102004. https://doi.org/10.1103/ PhysRevD.76.102004. arXiv:0706.4437

Connaughton V et al (2016) Fermi GBM observations of LIGO gravitational wave event GW150914. Astrophys J Lett 826:L6. https://doi.org/10.3847/2041-8205/826/1/L6. arXiv:1602.03920

Copperwheat CM et al (2016) Liverpool Telescope follow-up of candidate electromagnetic counterparts during the first run of Advanced LIGO. Mon Not R Astron Soc 462:3528. https://doi.org/10.1093/ mnras/stw1849. arXiv:1606.04574

Cornish NJ, Littenberg TB (2015) BayesWave: Bayesian Inference for Gravitational Wave Bursts and Instrument Glitches. Class Quantum Grav 32:135012. https://doi.org/10.1088/0264-9381/32/13/135012. arXiv: 1410.3835

Corsi A et al (2017) iPTF17cw: An engine-driven supernova candidate discovered independent of a gammaray trigger. Astrophys J 847:54. https://doi.org/10.3847/1538-4357/aa85e5. arXiv:1706.00045

Coulter DA et al (2017) Swope Supernova Survey 2017a (SSS17a), the optical counterpart to a gravitational wave source. Science 358(6370):1556-1558. https://doi.org/10.1126/science.aap9811. arXiv: 1710.05452

Cowperthwaite PS et al (2016) A DECam search for an optical counterpart to the LIGO gravitational wave event GW151226. Astrophys J Lett 826:L29. https://doi.org/10.3847/2041-8205/826/2/L29. arXiv: 1606.04538

Creminelli P, Vernizzi F (2017) Dark energy after GW170817 and GRB170817A. Phys Rev Lett 119:251302. https://doi.org/10.1103/PhysRevLett.119.251302. arXiv:1710.05877

Cutler C, Flanagan EE (1994) Gravitational waves from merging compact binaries: how accurately can one extract the binary's parameters from the inspiral wave form? Phys Rev D 49:2658-2697. https://doi. org/10.1103/PhysRevD.49.2658. arXiv:gr-qc/9402014

Dai L, McKinney JC, Miller MC (2017) Energetic constraints on electromagnetic signals from double black hole mergers. Mon Not R Astron Soc Lett 470:L92. https://doi.org/10.1093/mnras1/slx086. arXiv: 1611.00764

Dal Canton T, Harry IW (2017) Designing a template bank to observe compact binary coalescences in Advanced LIGO's second observing run. ArXiv e-prints arXiv:1705.01845

Dal Canton T, Lundgren AP, Nielsen AB (2015) Impact of precession on aligned-spin searches for neutron-star-black-hole binaries. Phys Rev D 91:062010. https://doi.org/10.1103/PhysRevD.91. 062010. arXiv:1411.6815

Dal Canton T et al (2014) Implementing a search for aligned-spin neutron star-black hole systems with advanced ground based gravitational wave detectors. Phys Rev D 90:082004. https://doi.org/10.1103/ PhysRevD.90.082004. arXiv:1405.6731

D'Avanzo P et al (2018) Evidence for a decreasing X-ray afterglow emission of GW170817A and GRB 170817A in XMM-Newton. ArXiv e-prints arXiv:1801.06164

Daw EJ, Giaime JA, Lormand D, Lubinski M, Zweizig J (2004) Long term study of the seismic environment at LIGO. Class Quantum Grav 21:2255-2273. https://doi.org/10.1088/0264-9381/21/9/003. arXiv:gr-qc/0403046

de Mink SE, Belczynski K (2015) Merger rates of double neutron stars and stellar origin black holes: the impact of initial conditions on binary evolution predictions. Astrophys J 814:58. https://doi.org/10. 1088/0004-637X/814/1/58. arXiv:1506.03573

de Mink SE, King A (2017) Electromagnetic signals following stellar-mass black hole mergers. Astrophys J Lett 839:L7. https://doi.org/10.3847/2041-8213/aa67f3. arXiv:1703.07794

Díaz MC et al (2016) GW150914: First search for the electromagnetic counterpart of a gravitational-wave event by the TOROS collaboration. Astrophys J Lett 828:L16. https://doi.org/10.3847/2041-8205/ 828/2/L16. arXiv:1607.07850 
Dimmelmeier H, Ott C, Marek A, Janka HT (2008) The gravitational wave burst signal from core collapse of rotating stars. Phys Rev D 78:064056. https://doi.org/10.1103/PhysRevD.78.064056. arXiv:0806.4953

Dominik M, Berti E, O’Shaughnessy R, Mandel I, Belczynski K, Fryer C, Holz DE, Bulik T, Pannarale F (2015) Double compact objects III: gravitational wave detection rates. Astrophys J 806:263. https:// doi.org/10.1088/0004-637X/806/2/263. arXiv:1405.7016

Dooley KL et al (2016) GEO 600 and the GEO-HF upgrade program: successes and challenges. Class Quantum Grav 33:075009. https://doi.org/10.1088/0264-9381/33/7/075009. arXiv:1510.00317

Effler A, Schofield RMS, Frolov VV, González G, Kawabe K, Smith JR, Birch J, McCarthy R (2015) Environmental Influences on the LIGO gravitational wave detectors during the 6th science run. Class Quantum Grav 32:035017. https://doi.org/10.1088/0264-9381/32/3/035017. arXiv:1409.5160

Eldridge JJ, Stanway ER, Xiao L, McClelland LAS, Taylor G, Ng M, Greis SML, Bray JC (2017) Binary population and spectral synthesis version 2.1: construction, observational verification, and new results. Publ Astron Soc Austral 34:e058. https://doi.org/10.1017/pasa.2017.51. arXiv:1710.02154

Essick R, Vitale S, Katsavounidis E, Vedovato G, Klimenko S (2015) Localization of short duration gravitational-wave transients with the early Advanced LIGO and Virgo detectors. Astrophys J 800:81. https://doi.org/10.1088/0004-637X/800/2/81. arXiv:1409.2435

Evans P et al (2012) Swift follow-up observations of candidate gravitational-wave transient events. Astrophys J Suppl 203:28. https://doi.org/10.1088/0067-0049/203/2/28. arXiv:1205.1124

Evans PA, Osborne JP, Kennea JA, Campana S, O’Brien PT, Tanvir NR, Racusin JL, Burrows DN, Cenko SB, Gehrels N (2016a) Optimisation of the Swift X-ray follow-up of Advanced LIGO and Virgo gravitational wave triggers in 2015-16. Mon Not R Astron Soc 455:1522-1537. https://doi.org/10. 1093/mnras/stv2213. arXiv:1506.01624

Evans PA et al (2016b) Swift follow-up of gravitational wave triggers: results from the first aLIGO run and optimisation for the future. Mon Not R Astron Soc 462:1591-1602. https://doi.org/10.1093/mnras/ stw1746. arXiv:1606.05001

Evans PA et al (2016c) Swift follow-up of the gravitational wave source GW150914. Mon Not R Astron Soc Lett 460:L40. https://doi.org/10.1093/mnrasl/slw065. arXiv:1602.03868

Ezquiaga JM, Zumalacárregui M (2017) Dark Energy After GW170817: dead ends and the road ahead. Phys Rev Lett 119:251304. https://doi.org/10.1103/PhysRevLett.119.251304. arXiv:1710.05901

Fairhurst S (2009) Triangulation of gravitational wave sources with a network of detectors. New J Phys 11:123006. https://doi.org/10.1088/1367-2630/11/12/123006, [Erratum: New J. Phys. 13:069602(2011)]. arXiv:0908.2356

Fairhurst S (2011) Source localization with an advanced gravitational wave detector network. Class Quantum Grav 28:105021. https://doi.org/10.1088/0264-9381/28/10/105021. arXiv:1010.6192

Fairhurst S (2017) Localization of transient gravitational wave sources: beyond triangulation. ArXiv e-prints arXiv: 1712.04724

Fan X, Messenger C, Heng IS (2014) A Bayesian approach to multi-messenger astronomy: Identification of gravitational-wave host galaxies. Astrophys J 795:43. https://doi.org/10.1088/0004-637X/795/1/ 43. arXiv:1406.1544

Farr B et al (2016) Parameter estimation on gravitational waves from neutron-star binaries with spinning components. Astrophys J 825:116. https://doi.org/10.3847/0004-637X/825/2/116. arXiv:1508.05336

Finn L, Chernoff D (1993) Observing binary inspiral in gravitational radiation: one interferometer. Phys Rev D 47:2198-2219. https://doi.org/10.1103/PhysRevD.47.2198. arXiv:gr-qc/9301003

Gaebel SM, Veitch J (2017) How would GW150914 look with future gravitational wave detector networks? Class Quant Grav 34:174003. https://doi.org/10.1088/1361-6382/aa82d9. arXiv:1703.08988

Gando A et al (2016) Search for electron antineutrinos associated with gravitational wave events GW150914 and GW151226 using KamLAND. Astrophys J Lett 829:L34. https://doi.org/10.3847/2041-8205/829/ 2/L34. arXiv:1606.07155

Gehrels N, Cannizzo JK, Kanner J, Kasliwal MM, Nissanke S, Singer LP (2016) Galaxy strategy for LIGOVirgo gravitational wave counterpart searches. Astrophys J 820:136. https://doi.org/10.3847/0004637X/820/2/136. arXiv:1508.03608

Ghosh S, Bloemen S, Nelemans G, Groot PJ, Price LR (2016) Tiling strategies for optical follow-up of gravitational-wave triggers by telescopes with a wide field of view. Astron Astrophys 592:A82. https:// doi.org/10.1051/0004-6361/201527712. arXiv:1511.02673 
Goldstein A et al (2017a) An ordinary short gamma-ray burst with extraordinary implications: Fermi-GBM detection of GRB 170817A. Astrophys J Lett 848:L14. https://doi.org/10.3847/2041-8213/aa8f41. arXiv: 1710.05446

Goldstein A et al (2017b) Fermi observations of the LIGO event GW170104. Astrophys J Lett 846:L5. https://doi.org/10.3847/2041-8213/aa8319. arXiv:1706.00199

Grote $\mathrm{H}$ et al (2013) First long-term application of squeezed states of light in a gravitational-wave observatory. Phys Rev Lett 110:181101. https://doi.org/10.1103/PhysRevLett.110.181101. arXiv:1302.2188

Grover K, Fairhurst S, Farr BF, Mandel I, Rodriguez C, Sidery T, Vecchio A (2014) Comparison of gravitational wave detector network sky localization approximations. Phys Rev D 89:042004. https://doi. org/10.1103/PhysRevD.89.042004. arXiv:1310.7454

Haggard D, Nynka M, Ruan JJ, Kalogera V, Bradley Cenko S, Evans P, Kennea JA (2017) A deep Chandra X-ray study of neutron star coalescence GW170817. Astrophys J Lett 848:L25. https://doi.org/10. 3847/2041-8213/aa8ede. arXiv: 1710.05852

Hallinan G et al (2017) A radio counterpart to a neutron star merger. Science 358(6370):1579-1583. https:// doi.org/10.1126/science.aap9855. arXiv:1710.05435

Hanna C, Mandel I, Vousden W (2014) Utility of galaxy catalogs for following up gravitational waves from binary neutron star mergers with wide-field telescopes. Astrophys J 784:8. https://doi.org/10.1088/ 0004-637X/784/1/8. arXiv:1312.2077

Harry GM (2010) Advanced LIGO: the next generation of gravitational wave detectors. Class Quantum Grav 27:084006. https://doi.org/10.1088/0264-9381/27/8/084006

Harry I, Privitera S, Bohé A, Buonanno A (2016) Searching for gravitational waves from compact binaries with precessing spins. Phys Rev D 94:024012. https://doi.org/10.1103/PhysRevD.94.024012. arXiv: 1603.02444

Harry IW, Allen B, Sathyaprakash BS (2009) A stochastic template placement algorithm for gravitational wave data analysis. Phys Rev D 80:104014. https://doi.org/10.1103/PhysRevD.80.104014. arXiv:0908.2090

Harry IW et al (2014) Investigating the effect of precession on searches for neutron-star-black-hole binaries with Advanced LIGO. Phys Rev D 89:024010. https://doi.org/10.1103/PhysRevD.89.024010. arXiv: 1307.3562

Hild S et al (2011) Sensitivity studies for third-generation gravitational wave observatories. Class Quantum Grav 28:094013. https://doi.org/10.1088/0264-9381/28/9/094013. arXiv:1012.0908

Hild S et al (2012) LIGO 3 Strawman Design, Team Red. Tech. Rep. LIGO-T1200046-v1, LIGO, Pasadena, CA. https://dcc.ligo.org/LIGO-T1200046/public

Hurley K et al (2016) The interplanetary network response to LIGO GW150914. Astrophys J Lett 829:L12. https://doi.org/10.3847/2041-8205/829/1/L12

Iyer B et al (2011) LIGO-India. Technical report M1100296-v2, IndIGO, India. https://dcc.ligo.org/LIGOM1100296/public

Janiuk A, Bejger M, Charzyński S, Sukova P (2017) On the possible gamma-ray burst-gravitational wave association in GW150914. New Astron 51:7-14. https://doi.org/10.1016/j.newast.2016.08.002. arXiv: 1604.07132

Jaranowski P, Królak A (2012) Gravitational-wave data analysis. Formalism and sample applications: the Gaussian case. Living Rev Relativ 15:4. https://doi.org/10.12942/lrr-2012-4. arXiv:0711.1115

Kanner JB et al (2016) Leveraging waveform complexity for confident detection of gravitational waves. Phys Rev D 93:022002. https://doi.org/10.1103/PhysRevD.93.022002. arXiv:1509.06423

Kasliwal MM, Nissanke S (2014) On discovering electromagnetic emission from neutron star mergers: the early years of two gravitational wave detectors. Astrophys J Lett 789:L5. https://doi.org/10.1088/ 2041-8205/789/1/L5. arXiv:1309.1554

Kasliwal MM et al (2016) iPTF Search for an Optical Counterpart to Gravitational Wave Trigger GW150914. Astrophys J Lett 824:L24. https://doi.org/10.3847/2041-8205/824/2/L24. arXiv:1602.08764

Kasliwal MM et al (2017) Illuminating gravitational waves: a concordant picture of photons from a neutron star merger. Science https://doi.org/10.1126/science.aap9455. arXiv:1710.05436

Kawai N, Negoro H, Serino M, Mihara T, Tanaka K, Masumitsu T, Nakahira S (2017) X-ray upper limits of GW150914 with MAXI. Publ Astron Soc Jpn 69:84. https://doi.org/10.1093/pasj/psx085. arXiv: 1708.01342

Khan S et al (2016) Frequency-domain gravitational waves from non-precessing black-hole binaries. II. A phenomenological model for the advanced detector era. Phys Rev D 93:044007. https://doi.org/10. 1103/PhysRevD.93.044007. arXiv:1508.07253 
Kim C, Perera BBP, McLaughlin MA (2013) Implications of PSR J0737-3039B for the Galactic NSNS binary merger rate. Mon Not R Astron Soc 448:928-938. https://doi.org/10.1093/mnras/stu2729. arXiv: 1308.4676

Klimenko S, Mohanty S, Rakhmanov M, Mitselmakher G (2005) Constraint likelihood analysis for a network of gravitational wave detectors. Phys Rev D 72:122002. https://doi.org/10.1103/PhysRevD. 72.122002. arXiv:gr-qc/0508068

Klimenko S, Yakushin I, Mercer A, Mitselmakher G (2008) Coherent method for detection of gravitational wave bursts. Class Quantum Grav 25:114029. https://doi.org/10.1088/0264-9381/25/11/ 114029. arXiv:0802.3232

Klimenko S, Vedovato G, Drago M, Mazzolo G, Mitselmakher G, Pankow C, Prodi G, Re V, Salemi F, Yakushin I (2011) Localization of gravitational wave sources with networks of advanced detectors. Phys Rev D 83:102001. https://doi.org/10.1103/PhysRevD.83.102001. arXiv:1101.5408

Klimenko S et al (2016) Method for detection and reconstruction of gravitational wave transients with networks of advanced detectors. Phys Rev D 93:042004. https://doi.org/10.1103/PhysRevD.93.042004. arXiv: 1511.05999

Kruckow MU, Tauris TM, Langer N, Kramer M, Izzard RG (2018) Progenitors of gravitational wave mergers: binary evolution with the stellar grid based code ComBinE. ArXiv e-prints arXiv:1801.05433

Li X, Zhang FW, Yuan Q, Jin ZP, Fan YZ, Liu SM, Wei DM (2016) Implications of the tentative association between GW150914 and a Fermi-GBM transient. Astrophys J Lett 827:L16. https://doi.org/10.3847/ 2041-8205/827/1/L16. arXiv:1602.04460

Li X, Hu YM, Jin ZP, Fan YZ, Wei DM (2017) Neutron star-black hole coalescence rate inferred from macronova/kilonova observations. Astrophys J Lett 844:L22. https://doi.org/10.3847/2041-8213/ aa7fb2. arXiv: 1611.01760

Lindblom L, Owen BJ, Brown DA (2008) Model waveform accuracy standards for gravitational wave data analysis. Phys Rev D 78:124020. https://doi.org/10.1103/PhysRevD.78.124020. arXiv:0809.3844

Lipunov VM et al (2017a) First gravitational-wave burst GW150914: MASTER optical follow-up observations. Mon Not R Astron Soc 465:3656. https://doi.org/10.1093/mnras/stw2669. arXiv:1605.01607

Lipunov VM et al (2017b) MASTER optical detection of the first LIGO/Virgo neutron star binary merger GW170817. Astrophys J 850:L1. https://doi.org/10.3847/2041-8213/aa92c0. arXiv:1710.05461

Littenberg TB, Cornish NJ (2015) Bayesian inference for spectral estimation of gravitational wave detector noise. Phys Rev D 91:084034. https://doi.org/10.1103/PhysRevD.91.084034. arXiv:1410.3852

Loeb A (2016) Electromagnetic counterparts to black hole mergers detected by LIGO. Astrophys J Lett 819:L21. https://doi.org/10.3847/2041-8205/819/2/L21. arXiv:1602.04735

Lück H et al (2010) The upgrade of GEO600. J Phys Conf Ser 228:012012. https://doi.org/10.1088/17426596/228/1/012012. arXiv:1004.0339

Lyman JD et al (2018) The optical afterglow of the short gamma-ray burst associated with GW170817. ArXiv e-prints arXiv:1801.02669

Lynch R, Vitale S, Essick R, Katsavounidis E, Robinet F (2017) Information-theoretic approach to the gravitational-wave burst detection problem. Phys Rev D 95:104046. https://doi.org/10.1103/ PhysRevD.95.104046. arXiv:1511.05955

Lyutikov M (2016) Fermi GBM signal contemporaneous with GW150914 - an unlikely association. ArXiv e-prints arXiv: 1602.07352

Mandel I, O'Shaughnessy R (2010) Compact binary coalescences in the band of ground-based gravitationalwave detectors. Class Quantum Grav 27:114007. https://doi.org/10.1088/0264-9381/27/11/114007. arXiv:0912.1074

Margutti R et al (2017) The electromagnetic counterpart of the binary neutron star merger LIGO/VIRGO GW170817. V. Rising X-ray emission from an off-axis jet. Astrophys J Lett 848:L20. https://doi.org/ 10.3847/2041-8213/aa9057. arXiv:1710.05431

Margutti R et al (2018) The Binary Neutron Star event LIGO/VIRGO GW170817 a hundred days after merger: synchrotron emission across the electromagnetic spectrum. ArXiv e-prints arXiv:1801.03531

McCully C et al (2017) The rapid reddening and featureless optical spectra of the optical counterpart of GW170817, AT 2017gfo, during the first four days. Astrophys J Lett 848:L32. https://doi.org/10.3847/ 2041-8213/aa9111. arXiv:1710.05853

Messick C et al (2017) Analysis framework for the prompt discovery of compact binary mergers in gravitational-wave data. Phys Rev D 95:042001. https://doi.org/10.1103/PhysRevD.95.042001. arXiv: 1604.04324 
Metzger BD (2017) Kilonovae. Living Rev Relativ 20:3. https://doi.org/10.1007/s41114-017-0006-z. arXiv: 1610.09381

Metzger BD, Berger E (2012) What is the most promising electromagnetic counterpart of a neutron star binary merger? Astrophys J 746:48. https://doi.org/10.1088/0004-637X/746/1/48. arXiv:1108.6056

Miller J et al (2015) Prospects for doubling the range of Advanced LIGO. Phys Rev D 91:062005. https:// doi.org/10.1103/PhysRevD.91.062005. arXiv:1410.5882

Mooley KP et al (2018) A mildly relativistic wide-angle outflow in the neutron star merger GW170817. Nature 554(7691):207. https://doi.org/10.1038/nature25452. arXiv:1711.11573

Morokuma T et al (2016) J-GEM follow-up observations to search for an optical counterpart of the first gravitational wave source GW150914. Publ Astron Soc Jpn 68:L9. https://doi.org/10.1093/pasj/psw061. arXiv: 1605.03216

Morsony BJ, Workman JC, Ryan DM (2016) Modeling the afterglow of the possible Fermi-GBM event associated with GW150914. Astrophys J Lett 825:L24. https://doi.org/10.3847/2041-8205/825/2/ L24. arXiv:1602.05529

Murase K, Kashiyama K, Mészáros P, Shoemaker I, Senno N (2016) Ultrafast outflows from black hole mergers with a minidisk. Astrophys J Lett 822:L9. https://doi.org/10.3847/2041-8205/822/1/L9. arXiv: 1602.06938

Nicholl M et al (2017) The electromagnetic counterpart of the binary neutron star merger LIGO/VIRGO GW170817 III. Optical and UV spectra of a blue kilonova from fast polar ejecta. Astrophys J Lett 848:L18. https://doi.org/10.3847/2041-8213/aa9029. arXiv:1710.05456

Nishizawa A, Berti E, Klein A, Sesana A (2016a) eLISA eccentricity measurements as tracers of binary black hole formation. Phys Rev D 94:064020. https://doi.org/10.1103/PhysRevD.94.064020. arXiv: 1605.01341

Nishizawa A, Sesana A, Berti E, Klein A (2016b) Constraining stellar binary black hole formation scenarios with eLISA eccentricity measurements. Mon Not R Astron Soc 465:4375. https://doi.org/10.1093/ mnras/stw2993. arXiv:1606.09295

Nissanke S, Holz DE, Hughes SA, Dalal N, Sievers JL (2010) Exploring short gamma-ray bursts as gravitational-wave standard sirens. Astrophys J 725:496-514. https://doi.org/10.1088/0004-637X/ 725/1/496. arXiv:0904.1017

Nissanke S, Sievers J, Dalal N, Holz D (2011) Localizing compact binary inspirals on the sky using groundbased gravitational wave interferometers. Astrophys J 739:99. https://doi.org/10.1088/0004-637X/ 739/2/99. arXiv:1105.3184

Nissanke S, Kasliwal M, Georgieva A (2013) Identifying elusive electromagnetic counterparts to gravitational wave mergers: an end-to-end simulation. Astrophys J 767:124. https://doi.org/10.1088/0004637X/767/2/124. arXiv:1210.6362

Nitz AH, Dent T, Dal Canton T, Fairhurst S, Brown DA (2017) Detecting binary compact-object mergers with gravitational waves: Understanding and Improving the sensitivity of the PyCBC search. Astrophys J 849:118. https://doi.org/10.3847/1538-4357/aa8f50. arXiv:1705.01513

Nitz AH et al (2013) Accuracy of gravitational waveform models for observing neutron-star-black-hole binaries in Advanced LIGO. Phys Rev D 88:124039. https://doi.org/10.1103/PhysRevD.88.124039. arXiv: 1307.1757

Ott C (2009) The gravitational wave signature of core-collapse supernovae. Class Quantum Grav 26:063001. https://doi.org/10.1088/0264-9381/26/6/063001. arXiv:0809.0695

Ott C, Reisswig C, Schnetter E, O’Connor E, Sperhake U, Löffler F, Diener P, Abdikamalov E, Hawke I, Burrows A (2011) Dynamics and gravitational wave signature of collapsar formation. Phys Rev Lett 106:161103. https://doi.org/10.1103/PhysRevLett.106.161103. arXiv:1012.1853

Owen BJ (1996) Search templates for gravitational waves from inspiraling binaries: choice of template spacing. Phys Rev D 53:6749-6761. https://doi.org/10.1103/PhysRevD.53.6749. arXiv:gr-qc/9511032

Owen BJ, Sathyaprakash B (1999) Matched filtering of gravitational waves from inspiraling compact binaries: computational cost and template placement. Phys Rev D 60:022002. https://doi.org/10.1103/ PhysRevD.60.022002. arXiv:gr-qc/9808076

Palliyaguru NT et al (2016) Radio follow-up of gravitational wave triggers during advanced LIGO O1. Astrophys J Lett 829:L28. https://doi.org/10.3847/2041-8205/829/2/L28. arXiv:1608.06518

Pan Y et al (2014) Inspiral-merger-ringdown waveforms of spinning, precessing black-hole binaries in the effective-one-body formalism. Phys Rev D 89:084006. https://doi.org/10.1103/PhysRevD.89.084006. arXiv: 1307.6232 
Pankow C, Chase EA, Coughlin S, Zevin M, Kalogera V (2018) Improvements in gravitational-wave sky localization with expanded networks of interferometers. Astrophys J Lett 854(2):L25. https://doi.org/ 10.3847/2041-8213/aaacd4. arXiv:1801.02674

Paschalidis V (2017) General relativistic simulations of compact binary mergers as engines of short gamma-ray bursts. Class Quantum Grav 34:084002. https://doi.org/10.1088/1361-6382/aa61ce. arXiv:1611.01519

Patricelli B, Stamerra A, Razzano M, Pian E, Cella G (2018) Searching for Gamma-Ray counterparts to Gravitational Waves from merging binary neutron stars with the Cherenkov telescope array. ArXiv e-prints arXiv:1801.05167

Patricelli B et al (2016) Prospects for joint observations of gravitational waves and gamma rays from merging neutron star binaries. J Cosmol Astropart Phys 1611:056. https://doi.org/10.1088/1475-7516/2016/ 11/056. arXiv:1606.06124

Perna R, Lazzati D, Giacomazzo B (2016) Short gamma-ray bursts from the merger of two black holes. Astrophys J Lett 821:L18. https://doi.org/10.3847/2041-8205/821/1/L18. arXiv:1602.05140

Pian E et al (2017) Spectroscopic identification of r-process nucleosynthesis in a double neutron star merger. Nature 551:67-70. https://doi.org/10.1038/nature24298. arXiv:1710.05858

Pitkin M, Reid S, Rowan S, Hough J (2011) Gravitational wave detection by interferometry (ground and space). Living Rev Relativ 14:5. https://doi.org/10.12942/lrr-2011-5. arXiv:1102.3355

Pooley D, Kumar P, Wheeler JC (2017) GW170817 most likely made a black hole. ArXiv e-prints arXiv: 1712.03240

Privitera S, Mohapatra SRP, Ajith P, Cannon K, Fotopoulos N, Frei MA, Hanna C, Weinstein AJ, Whelan JT (2014) Improving the sensitivity of a search for coalescing binary black holes with nonprecessing spins in gravitational wave data. Phys Rev D 89:024003. https://doi.org/10.1103/PhysRevD.89.024003. arXiv: 1310.5633

Prix R (2007) Template-based searches for gravitational waves: efficient lattice covering of flat parameter spaces. Class Quantum Grav 24:S481-S490. https://doi.org/10.1088/0264-9381/24/19/S11. arXiv:0707.0428

Punturo M et al (2010) The Einstein telescope: a third-generation gravitational wave observatory. Class Quantum Grav 27:194002. https://doi.org/10.1088/0264-9381/27/19/194002

Pürrer M (2014) Frequency domain reduced order models for gravitational waves from aligned-spin compact binaries. Class Quantum Grav 31:195010. https://doi.org/10.1088/0264-9381/31/19/195010. arXiv: 1402.4146

Racusin JL et al (2017) Searching the gamma-ray sky for counterparts to gravitational wave sources: Fermi GBM and LAT observations of LVT151012 and GW151226. Astrophys J 835:82. https://doi.org/10. 3847/1538-4357/835/1/82. arXiv:1606.04901

Rana J, Singhal A, Gadre B, Bhalerao V, Bose S (2017) An enhanced method for scheduling observations of large sky error regions for finding optical counterparts to transients. Astrophys J 838:108. https:// doi.org/10.3847/1538-4357/838/2/108. arXiv:1603.01689

Read JS et al (2013) Matter effects on binary neutron star waveforms. Phys Rev D 88:044042. https://doi. org/10.1103/PhysRevD.88.044042. arXiv:1306.4065

Riess AG et al (2016) A 2.4\% determination of the local value of the hubble constant. Astrophys J 826:56. https://doi.org/10.3847/0004-637X/826/1/56. arXiv:1604.01424

Rodriguez CL, Morscher M, Pattabiraman B, Chatterjee S, Haster CJ, Rasio FA (2015) Binary black hole mergers from globular clusters: implications for Advanced LIGO. Phys Rev Lett 115:051101. https:// doi.org/10.1103/PhysRevLett.115.051101. arXiv:1505.00792

Rodriguez CL et al (2014) Basic parameter estimation of binary neutron star systems by the Advanced LIGO/Virgo network. Astrophys J 784:119. https://doi.org/10.1088/0004-637X/784/2/119. arXiv: 1309.3273

Rosswog S et al (2017) Detectability of compact binary merger macronovae. Class Quantum Grav 34:104001. https://doi.org/10.1088/1361-6382/aa68a9. arXiv:1611.09822

Ruan JJ, Nynka M, Haggard D, Kalogera V, Evans P (2018) Brightening X-ray emission from GW170817/GRB170817A: further evidence for an outflow. Astrophys J Lett 853(1):L4. https://doi. org/10.3847/2041-8213/aaa4f3. arXiv:1712.02809

Ryan G, MacFadyen A (2017) Minidisks in binary black hole accretion. Astrophys J 835:199. https://doi. org/10.3847/1538-4357/835/2/199. arXiv:1611.00341 
Sakstein J, Jain B (2017) Implications of the neutron star merger GW170817 for cosmological scalar-tensor theories. Phys Rev Lett 119:251303. https://doi.org/10.1103/PhysRevLett.119.251303. arXiv: 1710.05893

Salafia OS, Colpi M, Branchesi M, Chassande-Mottin E, Ghirlanda G, Ghisellini G, Vergani S (2017) Where and when: optimal scheduling of the electromagnetic follow-up of gravitational-wave events based on counterpart lightcurve models. Astrophys J 846:62. https://doi.org/10.3847/1538-4357/aa850e. arXiv: 1704.05851

Sathyaprakash B, Schutz BF (2009) Physics, astrophysics and cosmology with gravitational waves. Living Rev Relativ 12:2. https://doi.org/10.12942/1rr-2009-2. arXiv:0903.0338

Sathyaprakash B et al (2012) Scientific objectives of Einstein telescope. Class Quantum Grav 29:124013. https://doi.org/10.1088/0264-9381/29/12/124013. arXiv:1206.0331

Sathyaprakash BS, Dhurandhar SV (1991) Choice of filters for the detection of gravitational waves from coalescing binaries. Phys Rev D 44:3819-3834. https://doi.org/10.1103/PhysRevD.44.3819

Savchenko V et al (2016) INTEGRAL upper limits on gamma-ray emission associated with the gravitational wave event GW150914. Astrophys J Lett 820:L36. https://doi.org/10.3847/2041-8205/820/2/L36. arXiv: 1602.04180

Savchenko V et al (2017a) INTEGRAL detection of the first prompt gamma-ray signal coincident with the gravitational-wave event GW170817. Astrophys J Lett 848:L15. https://doi.org/10.3847/2041-8213/ aa8f94. arXiv: 1710.05449

Savchenko V et al (2017b) INTEGRAL observations of GW170104. Astrophys J Lett 846:L23. https://doi. org/10.3847/2041-8213/aa87ae. arXiv:1707.03719

Schmidt P, Ohme F, Hannam M (2015) Towards models of gravitational waveforms from generic binaries II: Modelling precession effects with a single effective precession parameter. Phys Rev D 91:024043. https://doi.org/10.1103/PhysRevD.91.024043. arXiv:1408.1810

Schnittman JD (2013) Astrophysics of super-massive black hole mergers. Class Quantum Grav 30:244007. https://doi.org/10.1088/0264-9381/30/24/244007. arXiv:1307.3542

Schutz BF (1986) Determining the Hubble constant from gravitational wave observations. Nature 323:310311. https://doi.org/10.1038/323310a0

Schutz BF (2011) Networks of gravitational wave detectors and three figures of merit. Class Quantum Grav 28:125023. https://doi.org/10.1088/0264-9381/28/12/125023. arXiv:1102.5421

Serino M, Kawai N, Negoro H, Mihara T, Masumitsu T, Nakahira S (2017) X-ray upper limits of GW151226 with MAXI. Publ Astron Soc Jpn 69:85. https://doi.org/10.1093/pasj/psx086. arXiv:1708.01352

Sesana A (2016) Prospects for multiband gravitational-wave astronomy after GW150914. Phys Rev Lett 116:231102. https://doi.org/10.1103/PhysRevLett.116.231102. arXiv:1602.06951

Shappee BJ et al (2017) Early spectra of the gravitational wave source GW170817: evolution of a neutron star merger. Science. https://doi.org/10.1126/science.aaq0186, arXiv:1710.05432

Sidery T et al (2014) Reconstructing the sky location of gravitational-wave detected compact binary systems: methodology for testing and comparison. Phys Rev D 89:084060. https://doi.org/10.1103/PhysRevD. 89.084060. arXiv: 1312.6013

Siebert MR et al (2017) The unprecedented properties of the first electromagnetic counterpart to a gravitational wave source. Astrophys J Lett 848:L26. https://doi.org/10.3847/2041-8213/aa905e. arXiv: 1710.05440

Singer LP, Price LR (2016) Rapid Bayesian position reconstruction for gravitational-wave transients. Phys Rev D 93:024013. https://doi.org/10.1103/PhysRevD.93.024013. arXiv:1508.03634

Singer LP et al (2014) The first two years of electromagnetic follow-up with Advanced LIGO and Virgo. Astrophys J 795:105. https://doi.org/10.1088/0004-637X/795/2/105. arXiv:1404.5623

Singer LP et al (2016a) Going the distance: mapping host galaxies of LIGO and Virgo sources in three dimensions using local cosmography and targeted follow-up. Astrophys J Lett 829:L15. https://doi. org/10.3847/2041-8205/829/1/L15. arXiv:1603.07333

Singer LP et al (2016b) Supplement: going the distance: mapping host galaxies of LIGO and Virgo sources in three dimensions using local cosmography and targeted follow-up. Astrophys J Suppl 226:10. https:// doi.org/10.3847/0067-0049/226/1/10. arXiv:1605.04242

Smartt SJ et al (2016a) A search for an optical counterpart to the gravitational wave event GW151226. Astrophys J Lett 827:L40. https://doi.org/10.3847/2041-8205/827/2/L40. arXiv:1606.04795

Smartt SJ et al (2016b) Pan-STARRS and PESSTO search for an optical counterpart to the LIGO gravitational wave source GW150914. Mon Not R Astron Soc 462:4094. https://doi.org/10.1093/mnras/ stw1893. arXiv:1602.04156 
Smartt SJ et al (2017) A kilonova as the electromagnetic counterpart to a gravitational-wave source. Nature 551(7678):75-79. https://doi.org/10.1038/nature24303. arXiv:1710.05841

Smith R, Field SE, Blackburn K, Haster CJ, Pürrer M, Raymond V, Schmidt P (2016) Fast and accurate inference on gravitational waves from precessing compact binaries. Phys Rev D 94:044031. https:// doi.org/10.1103/PhysRevD.94.044031. arXiv:1604.08253

Soares-Santos M et al (2016) A dark energy camera search for an optical counterpart to the first Advanced LIGO gravitational wave event GW150914. Astrophys J Lett 823:L33. https://doi.org/10.3847/20418205/823/2/L33. arXiv:1602.04198

Soares-Santos M et al (2017) The electromagnetic counterpart of the binary neutron star merger LIGO/Virgo GW170817 I. Discovery of the optical counterpart using the dark energy camera. Astrophys J Lett 848:L16. https://doi.org/10.3847/2041-8213/aa9059. arXiv:1710.05459

Somiya K (2012) Detector configuration of KAGRA: the Japanese cryogenic gravitational-wave detector. Class Quantum Grav 29:124007. https://doi.org/10.1088/0264-9381/29/12/124007. arXiv:1111.7185

Stalder B et al (2017) Observations of the GRB afterglow ATLAS17aeu and its possible association with GW170104. Astrophys J 850:149. https://doi.org/10.3847/1538-4357/aa95c1. arXiv:1706.00175

Staley A et al (2014) Achieving resonance in the Advanced LIGO gravitational-wave interferometer. Class Quantum Grav 31:245010. https://doi.org/10.1088/0264-9381/31/24/245010

Stone NC, Metzger BD, Haiman Z (2017) Assisted inspirals of stellar mass black holes embedded in AGN disks. Mon Not R Astron Soc 464:946-954. https://doi.org/10.1093/mnras/stw2260. arXiv: 1602.04226

Sutton P (2013) A rule of thumb for the detectability of gravitational-wave bursts. ArXiv e-prints arXiv: 1304.0210

Sutton PJ et al (2010) X-pipeline: an analysis package for autonomous gravitational-wave burst searches. New J Phys 12:053034. https://doi.org/10.1088/1367-2630/12/5/053034. arXiv:0908.3665

Tanvir NR et al (2017) The emergence of a lanthanide-rich kilonova following the merger of two neutron stars. Astrophys J Lett 848:L27. https://doi.org/10.3847/2041-8213/aa90b6. arXiv:1710.05455

Taracchini A et al (2014) Effective-one-body model for black-hole binaries with generic mass ratios and spins. Phys Rev D 89:061502. https://doi.org/10.1103/PhysRevD.89.061502. arXiv:1311.2544

Tavani M et al (2016) AGILE observations of the gravitational wave event GW150914. Astrophys J Lett 825:L4. https://doi.org/10.3847/2041-8205/825/1/L4. arXiv:1604.00955

Thrane E, Coughlin M (2013) Searching for gravitational-wave transients with a qualitative signal model: seedless clustering strategies. Phys Rev D 88:083010. https://doi.org/10.1103/PhysRevD.88.083010. arXiv: 1308.5292

Thrane E et al (2011) Long gravitational-wave transients and associated detection strategies for a network of terrestrial interferometers. Phys Rev D 83:083004. https://doi.org/10.1103/PhysRevD.83.083004. arXiv: 1012.2150

Thrane E, Mandic V, Christensen N (2015) Detecting very long-lived gravitational-wave transients lasting hours to weeks. Phys Rev S 91:104021. https://doi.org/10.1103/PhysRevD.91.104021. arXiv: 1501.06648

Troja E, Read AM, Tiengo A, Salvaterra R (2016) XMM-Newton Slew Survey observations of the gravitational wave event GW150914. Astrophys J Lett 822:L8. https://doi.org/10.3847/2041-8205/822/1/ L8. arXiv: 1603.06585

Troja E et al (2017) The X-ray counterpart to the gravitational wave event GW 170817. Nature 551:71-74. https://doi.org/10.1038/nature24290. arXiv:1710.05433

Usman SA, Nitz AH, Harry IW, Biwer CM, Brown DA, Cabero M, Capano CD, Dal Canton T, Dent T, Fairhurst S, Kehl MS, Keppel D, Krishnan B, Lenon A, Lundgren A, Nielsen AB, Pekowsky LP, Pfeiffer HP, Saulson PR, West M, Willis JL (2016) An improved pipeline to search for gravitational waves from compact binary coalescence. Class Quantum Grav 33:215004. https://doi.org/10.1088/ 0264-9381/33/21/215004. arXiv:1508.02357

van der Sluys MV, Roever C, Stroeer A, Christensen N, Kalogera V, Meyer R, Vecchio A (2008) Gravitational-wave astronomy with inspiral signals of spinning compact-object binaries. Astrophys J Lett 688:L61. https://doi.org/10.1086/595279. arXiv:0710.1897

Valenti S, Sand DJ, Yang S, Cappellaro E, Tartaglia L, Corsi A, Jha SW, Reichart DE, Haislip J, Kouprianov V (2017) The discovery of the electromagnetic counterpart of GW170817: Kilonova AT 2017gfo/DLT17ck. Astrophys J Lett 848:L24. https://doi.org/10.3847/2041-8213/aa8edf. arXiv: 1710.05854 
Vallisneri M, Kanner J, Williams R, Weinstein A, Stephens B (2015) The LIGO open science center. J Phys Conf Ser 610:012021. https://doi.org/10.1088/1742-6596/610/1/012021. arXiv:1410.4839

Vangioni E, Goriely S, Daigne F, François P, Belczynski K (2016) Cosmic neutron star merger rate and gravitational waves constrained by the r-process nucleosynthesis. Mon Not R Astron Soc 455:17-34. https://doi.org/10.1093/mnras/stv2296. arXiv:1501.01115

Vecchio A (2004) LISA observations of rapidly spinning massive black hole binary systems. Phys Rev D 70:042001. https://doi.org/10.1103/PhysRevD.70.042001. arXiv:astro-ph/0304051

Veitch J, Mandel I, Aylott B, Farr B, Raymond V, Rodriguez C, van der Sluys M, Kalogera V, Vecchio A (2012) Estimating parameters of coalescing compact binaries with proposed advanced detector networks. Phys Rev D 85:104045. https://doi.org/10.1103/PhysRevD.85.104045. arXiv:1201.1195

Veitch J, Raymond V, Farr B, Farr W, Graff P, Vitale S, Aylott B, Blackburn K, Christensen N, Coughlin M, Del Pozzo W, Feroz F, Gair J, Haster CJ, Kalogera V, Littenberg T, Mandel I, O’Shaughnessy R, Pitkin M, Rodriguez C, Röver C, Sidery T, Smith R, Van Der Sluys M, Vecchio A, Vousden W, Wade L (2015) Parameter estimation for compact binaries with ground-based gravitational-wave observations using the LALInference software library. Phys Rev D 91:042003. https://doi.org/10.1103/PhysRevD. 91.042003. arXiv: 1409.7215

Verrecchia F et al (2017) AGILE observations of the gravitational-wave source GW170104. Astrophys J Lett 847:L20. https://doi.org/10.3847/2041-8213/aa8224. arXiv:1706.00029

Villar VA et al (2017) The combined ultraviolet, optical, and near-infrared light curves of the kilonova associated with the binary neutron star merger GW170817: unified data set, analytic models, and physical implications. Astrophys J Lett 851:L21. https://doi.org/10.3847/2041-8213/aa9c84. arXiv: 1710.11576

Vinciguerra S, Veitch J, Mandel I (2017) Accelerating gravitational wave parameter estimation with multiband template interpolation. Class Quantum Grav 34:115006. https://doi.org/10.1088/1361-6382/ aa6d44. arXiv:1703.02062

Vitale S (2016) Multiband gravitational-wave astronomy: parameter estimation and tests of general relativity with space- and ground-based detectors. Phys Rev Lett 117:051102. https://doi.org/10.1103/ PhysRevLett.117.051102. arXiv:1605.01037

Vitale S, Zanolin M (2011) Application of asymptotic expansions for maximum likelihood estimators' errors to gravitational waves from binary mergers: the network case. Phys Rev D 84:104020. https:// doi.org/10.1103/PhysRevD.84.104020. arXiv:1108.2410

Vitale S, Del Pozzo W, Li TG, Van Den Broeck C, Mandel I, Aylott B, Veitch J (2012) Effect of calibration errors on Bayesian parameter estimation for gravitational wave signals from inspiral binary systems in the advanced detectors era. Phys Rev D 85:064034. https://doi.org/10.1103/PhysRevD.85.064034. arXiv: 1111.3044

Vitale S, Lynch R, Veitch J, Raymond V, Sturani R (2014) Measuring the spin of black holes in binary systems using gravitational waves. Phys Rev Lett 112:251101. https://doi.org/10.1103/PhysRevLett. 112.251101. arXiv: 1403.0129

Vitale S et al (2016) On similarity of binary black hole gravitational-wave skymaps: to observe or to wait? Mon Not R Astron Lett 466:L78. https://doi.org/10.1093/mnrasl/slw239. arXiv:1611.02438

Woosley SE (2016) The progenitor of GW150914. Astrophys J Lett 824:L10. https://doi.org/10.3847/20418205/824/1/L10. arXiv:1603.00511

Yakumin K et al (2010) Gravitational waves from core collapse supernovae. Class Quantum Grav 27:194005. https://doi.org/10.1088/0264-9381/27/19/194005. arXiv:1005.0779

Yamazaki R, Asano K, Ohira Y (2016) Electromagnetic afterglows associated with gamma-ray emission coincident with binary black hole merger event GW150914. Progr Theor Exp Phys 2016:051E01. https://doi.org/10.1093/ptep/ptw042. arXiv:1602.05050

Yang S, Valenti S, Cappellaro E, Sand DJ, Tartaglia L, Corsi A, Reichart DE, Haislip J, Kouprianov V (2017) An empirical limit on the kilonova rate from the DLT40 one day cadence Supernova Survey. Astrophys J Lett 851:L48. https://doi.org/10.3847/2041-8213/aaa07d. arXiv:1710.05864

Yoshida M et al (2017) J-GEM follow-up observations of the gravitational wave source GW151226. Publ Astron Soc Jpn 69:12. https://doi.org/10.1093/pasj/psw113. arXiv:1611.01588

Zhang BB et al (2018) A peculiar low-luminosity short gamma-ray burst from a double neutron star merger progenitor. Nature Commun 9:447. https://doi.org/10.1038/s41467-018-02847-3. arXiv:1710.05851

Publisher's Note Springer Nature remains neutral with regard to jurisdictional claims in published maps and institutional affiliations. 


\section{Affiliations}

B. P. Abbott ${ }^{1}$ - R. Abbott Ab $^{1}$ - T. D. Abbott ${ }^{2}$ - M. R. Abernathy ${ }^{3}$.

F. Acernese A,5 $^{\text {, K. Ackley }}{ }^{6}$ - C. Adams ${ }^{7}$. T. Adams ${ }^{8}$ - P. Addesso 9,10 .

R. X. Adhikari ${ }^{1}$ - V. B. Adya ${ }^{11}$. C. Affeldt ${ }^{11}$ - M. Agathos ${ }^{12}$.

K. Agatsuma 12 - N. Aggarwal $^{13}$ - O. D. Aguiar ${ }^{14}$ - L. Aiello 15,16 A. Ain ${ }^{17}$. P. Ajith ${ }^{18}$. T. Akutsu ${ }^{19}$ - B. Allen ${ }^{11,20,21}$ - A. Allocca ${ }^{22,23}$. P. A. Altin ${ }^{24}$.

A. Ananyeva ${ }^{1}$ - S. B. Anderson' ${ }^{1}$. W. G. Anderson ${ }^{20}$ - M. Ando 19,25,26 .

S. Appert ${ }^{1}$ - K. Arai ${ }^{1}$ - A. Araya ${ }^{27}$ - M. C. Araya ${ }^{1}$ - J. S. Areeda ${ }^{28}$.

N. Arnaud ${ }^{29}$ - K. G. Arun ${ }^{30}$ - H. Asada ${ }^{31}$ - S. Ascenzi ${ }^{16,32}$ - G. Ashton ${ }^{11}$.

Y. Aso ${ }^{19}$ - M. Ast A3 $^{33}$ - S. M. Aston ${ }^{7}$ - P. Astone As $^{34}$. S. Atsuta ${ }^{35}$.

P. Aufmuth ${ }^{21}$. C. Aulbert ${ }^{11}$ - A. Avila-Alvarez ${ }^{28}$ - K. Awai ${ }^{36}$ - S. Babak ${ }^{37}$.

P. Bacon ${ }^{38}$ - M. K. M. Bader ${ }^{12}$ - L. Baiotti ${ }^{39}$ - P. T. Baker ${ }^{40,41}$.

F. Baldaccini ${ }^{42,43}$ - G. Ballardin ${ }^{44}$ - S. W. Ballmer ${ }^{45}$ - J. C. Barayoga ${ }^{1}$.

S. E. Barclay ${ }^{46}$ - B. C. Barish ${ }^{1}$ - D. Barker ${ }^{47}$ - F. Barone B $^{4,5}$ - B. Barr B $^{46}$.

L. Barsotti ${ }^{13}$ - M. Barsuglia ${ }^{38}$. D. Barta ${ }^{48}$ - J. Bartlett ${ }^{47}$ - M. A. Barton ${ }^{19}$.

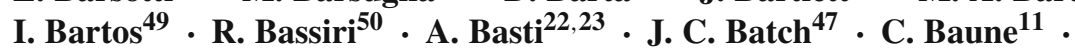

V. Bavigadda ${ }^{44}$. M. Bazzan ${ }^{51,52}$. B. Bécsy ${ }^{53}$. C. Beer ${ }^{11}$ - M. Bejger ${ }^{54}$.

I. Belahcene ${ }^{29}$ - M. Belgin 55 - A. S. Bell ${ }^{46}$ - B. K. Berger ${ }^{1}$ - G. Bergmann ${ }^{11}$.

C. P. L. Berry ${ }^{56}$ - D. Bersanetti ${ }^{57,58}$ - A. Bertolini ${ }^{12}$ - J. Betzwieser ${ }^{7}$.

S. Bhagwat ${ }^{45}$ - R. Bhandare ${ }^{59}$ - I. A. Bilenko ${ }^{60}$ - G. Billingsley ${ }^{1}$.

C. R. Billman ${ }^{6}$ - J. Birch ${ }^{7}$ - R. Birney ${ }^{61}$ - O. Birnholtz ${ }^{11} \cdot$ S. Biscans ${ }^{1,13}$.

A. Bisht ${ }^{21}$ - M. Bitossi ${ }^{44}$ - C. Biwer ${ }^{45}$ - M. A. Bizouard ${ }^{29}$.

J. K. Blackburn ${ }^{1}$ - J. Blackman ${ }^{62}$ - C. D. Blair ${ }^{63}$ - D. G. Blair ${ }^{63}$.

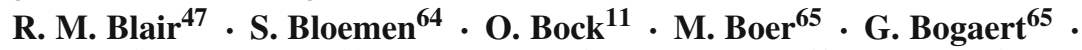

A. Bohe ${ }^{37}$ - F. Bondu ${ }^{66}$ - R. Bonnand ${ }^{8}$ - B. A. Boom ${ }^{12}$ - R. Bork ${ }^{1}$.

V. Boschi' 22,23 . S. Bose ${ }^{17,67}$ - Y. Bouffanais ${ }^{38}$ - A. Bozzi ${ }^{44}$.

C. Bradaschia ${ }^{23}$. P. R. Brady ${ }^{20}$. V. B. Braginsky*,60 - M. Branchesi ${ }^{68,69}$.

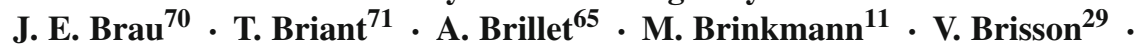

P. Brockill ${ }^{20}$ - J. E. Broida ${ }^{72}$ - A. F. Brooks ${ }^{1}$ - D. A. Brown ${ }^{45}$.

D. D. Brown ${ }^{56}$ - N. M. Brown ${ }^{13}$. S. Brunett ${ }^{1}$ - C. C. Buchanan 2 .

A. Buikema ${ }^{13}$ - T. Bulik ${ }^{73}$ - H. J. Bulten ${ }^{12,74}$ - A. Buonanno ${ }^{37,75}$.

D. Buskulic ${ }^{8}$ - C. Buy ${ }^{38}$ - R. L. Byer ${ }^{50}$ - M. Cabero ${ }^{11}$ - L. Cadonati ${ }^{55}$.

G. Cagnoli ${ }^{76,77}$ - C. Cahillane ${ }^{1}$ - J. Calderón Bustillo ${ }^{55}$ - T. A. Callister ${ }^{1}$.

E. Calloni ${ }^{5,78}$ - J. B. Camp ${ }^{79}$ - K. C. Cannon ${ }^{26}$ - H. Cao ${ }^{80}$ - J. Cao ${ }^{81}$.

C. D. Capano ${ }^{11}$ - E. Capocasa ${ }^{38}$ - F. Carbognani ${ }^{44}$. S. Caride ${ }^{82}$.

J. Casanueva Diaz ${ }^{29}$ - C. Casentini ${ }^{16,32}$ - S. Caudill ${ }^{20}$ - M. Cavaglià ${ }^{83}$.

F. Cavalier ${ }^{29}$ - R. Cavalieri $^{44}$ - G. Cella ${ }^{23}$ - C. B. Cepeda ${ }^{1}$.

L. Cerboni Baiardi ${ }^{68,69}$ - G. Cerretani ${ }^{22,23}$ - E. Cesarini ${ }^{16,32}$.

S. J. Chamberlin ${ }^{84}$ - M. Chan ${ }^{46} \cdot$ S. Chao ${ }^{85} \cdot$ P. Charlton ${ }^{86}$.

E. Chassande-Mottin ${ }^{38}$. B. D. Cheeseboro ${ }^{40,41}$ - H. Y. Chen ${ }^{87}$. Y. Chen ${ }^{62}$.

H.-P. Cheng ${ }^{6}$ - A. Chincarini ${ }^{58}$ - A. Chiummo ${ }^{44}$ - T. Chmiel ${ }^{88}$.

H. S. Cho ${ }^{89} \cdot$ M. Cho ${ }^{75}$ - J. H. Chow ${ }^{24} \cdot$ N. Christensen ${ }^{72} \cdot$ Q. Chu ${ }^{63}$.

A. J. K. Chua ${ }^{90}$ - S. Chua ${ }^{71}$ - S. Chung ${ }^{63}$ - G. Ciani ${ }^{6}$ - F. Clara ${ }^{47}$.

J. A. Clark ${ }^{55}$. F. Cleva ${ }^{65}$ - C. Cocchieri ${ }^{83}$. E. Coccia ${ }^{15,16}$.

P.-F. Cohadon ${ }^{71}$ - A. Colla ${ }^{34,91}$ - C. G. Collette ${ }^{92}$. L. Cominsky ${ }^{93}$. 
M. Constancio Jr. ${ }^{14}$ - L. Conti ${ }^{52}$ - S. J. Cooper ${ }^{56}$ - T. R. Corbitt ${ }^{2}$.

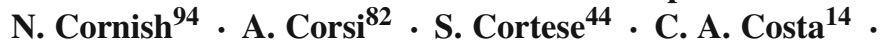

M. W. Coughlin ${ }^{72}$ - S. B. Coughlin ${ }^{95}$ - J.-P. Coulon ${ }^{65}$ - S. T. Countryman ${ }^{49}$.

P. Couvares ${ }^{1}$ - P. B. Covas $^{96}$ - E. E. Cowan ${ }^{55}$. D. M. Coward ${ }^{63}$.

M. J. Cowart ${ }^{7}$ - D. C. Coyne ${ }^{1}$ - R. Coyne ${ }^{82}$ - J. D. E. Creighton ${ }^{20}$.

T. D. Creighton' ${ }^{97}$ - J. Cripe ${ }^{2}$ - S. G. Crowder98 * T. J. Cullen ${ }^{28}$.

A. Cumming ${ }^{46}$. L. Cunningham ${ }^{46}$ - E. Cuoco ${ }^{44}$. T. Dal Canton ${ }^{79}$.

S. L. Danilishin ${ }^{46}$. S. D'Antonio ${ }^{16}$ - K. Danzmann 11,21 * A. Dasgupta ${ }^{99}$.

C. F. Da Silva Costa ${ }^{6}$. V. Dattilo ${ }^{44}$. I. Dave D9 $^{59}$ M. Davier ${ }^{29}$.

G. S. Davies ${ }^{46}$. D. Davis ${ }^{45}$ - E. J. Daw ${ }^{100}$ - B. Day ${ }^{55}$ - R. Day ${ }^{44}$. S. De D $^{45}$.

D. DeBra ${ }^{50}$ - G. Debreczeni ${ }^{48}$ - J. Degallaix ${ }^{76}$ - M. De Laurentis ${ }^{5,78}$.

S. Deléglise ${ }^{71}$ - W. Del Pozzo ${ }^{56}$ - T. Denker ${ }^{11}$ - T. Dent ${ }^{11}$ - V. Dergachev ${ }^{37}$.

R. De Rosa ${ }^{5,78}$ - R. T. DeRosa ${ }^{7}$ - R. DeSalvo 9,10,101 - R. C. Devine D0,41 $^{\text {. }}$

S. Dhurandhar ${ }^{17}$. M. C. Díaz ${ }^{97}$. L. Di Fiore ${ }^{5}$. M. Di Giovanni ${ }^{102,103}$.

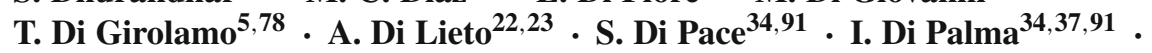

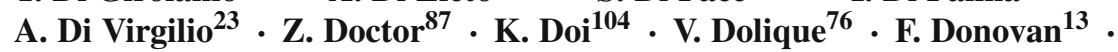

K. L. Dooley ${ }^{83}$. S. Doravari ${ }^{11}$ - I. Dorrington ${ }^{105}$ - R. Douglas ${ }^{46}$.

M. Dovale Álvarez ${ }^{56}$. T. P. Downes ${ }^{20}$ - M. Drago ${ }^{11}$ - R. W. P. Drever ${ }^{\sharp, 1}$.

J. C. Driggers ${ }^{47}$ - Z. Du ${ }^{81}$ - M. Ducrot ${ }^{8}$ - S. E. Dwyer ${ }^{47}$ - K. Eda Ed $^{26}$.

T. B. Edo ${ }^{100}$ - M. C. Edwards ${ }^{72}$ - A. Effler ${ }^{7}$ - H.-B. Eggenstein ${ }^{11}$.

P. Ehrens ${ }^{1}$ - J. Eichholz ${ }^{1}$ - S. S. Eikenberry6 ${ }^{6}$ R. A. Eisenstein ${ }^{13}$.

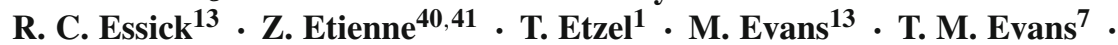

R. Everett - M. Factourovich $^{49}$ - V. Fafone $15,16,32$ - H. Fair ${ }^{45}$.

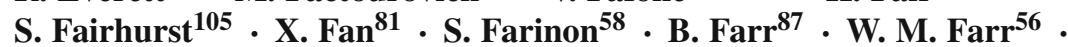

E. J. Fauchon-Jones ${ }^{105}$ - M. Favata ${ }^{106}$ - M. Fays ${ }^{105}$ - H. Fehrmann ${ }^{11}$.

M. M. Fejer ${ }^{50}$ - A. Fernández Galiana ${ }^{13}$ - I. Ferrante Fe,23 $^{22}$ E. C. Ferreira ${ }^{14}$.

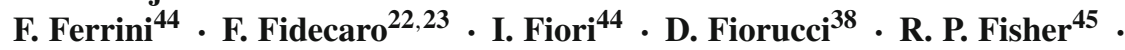

R. Flaminio ${ }^{19,76}$ - M. Fletcher ${ }^{46}$ - H. Fong ${ }^{107}$ - S. S. Forsyth ${ }^{55}$.

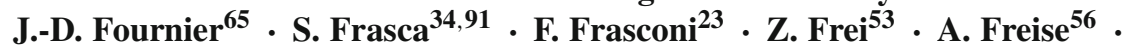

R. Frey ${ }^{70}$ - V. Frey ${ }^{29}$ - E. M. Fries ${ }^{1}$ - P. Fritschel ${ }^{13}$ - V. V. Frolov ${ }^{7}$. $^{1}$

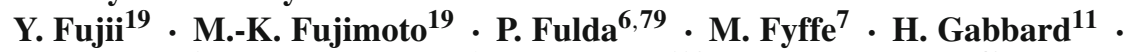

B. U. Gadre ${ }^{17}$ - S. M. Gaebel ${ }^{56}$. J. R. Gair ${ }^{108}$ - L. Gammaitoni ${ }^{42}$.

S. G. Gaonkar ${ }^{17}$ - F. Garufi ${ }^{5,78}$. G. Gaur ${ }^{109}$ - V. Gayathri ${ }^{110}$.

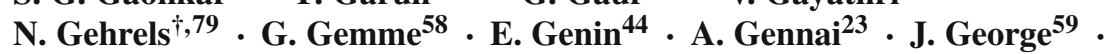

L. Gergely ${ }^{111}$ - V. Germain ${ }^{8}$ - S. Ghonge ${ }^{18}$ - Abhirup Ghosh ${ }^{18}$.

Archisman Ghosh ${ }^{12,18}$ - S. Ghosh ${ }^{12,64}$ - J. A. Giaime ${ }^{2,7}$ - K. D. Giardina ${ }^{7}$.

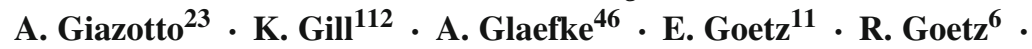

L. Gondan ${ }^{53}$. G. González ${ }^{2}$. J. M. Gonzalez Castro ${ }^{22,23}$.

A. Gopakumar ${ }^{113}$ - M. L. Gorodetsky ${ }^{60}$ - S. E. Gossan' ${ }^{1}$ M. Gosselin 44 .

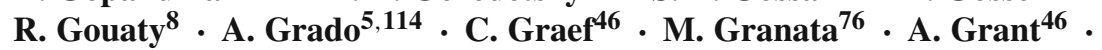

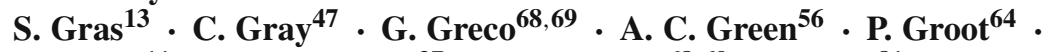

H. Grote ${ }^{11}$ - S. Grunewald ${ }^{37}$ - G. M. Guidi ${ }^{68,69}$ - X. Guo ${ }^{81}$ - A. Gupta ${ }^{17}$.

M. K. Gupta99 • K. E. Gushwa ${ }^{1}$ - E. K. Gustafson' ${ }^{1}$. R. Gustafson ${ }^{115}$.

J. J. Hacker ${ }^{28}$ - A. Hagiwara ${ }^{116}$ - B. R. Hall ${ }^{67}$ - E. D. Hall ${ }^{1}$.

G. Hammond ${ }^{46}$ - M. Haney ${ }^{113}$ - M. M. Hanke ${ }^{11}$ - J. Hanks ${ }^{47}$. C. Hanna ${ }^{84}$.

M. D. Hannam ${ }^{105}$ • J. Hanson ${ }^{7}$ - T. Hardwick ${ }^{2}$ - J. Harms H8,69 $^{\text {. }}$ 
G. M. Harry ${ }^{3}$ - I. W. Harry ${ }^{37}$ - M. J. Hart ${ }^{46}$ - M. T. Hartman6 ${ }^{6}$ C.-J. Haster 56,107 • K. Haughian ${ }^{46}$ - K. Hayama ${ }^{36}$ - J. Healy H17 $^{\text {. }}$

A. Heidmann ${ }^{71}$ - M. C. Heintze ${ }^{7}$ - H. Heitmann ${ }^{65}$ - P. Hello ${ }^{29}$.

G. Hemming ${ }^{44}$ - M. Hendry ${ }^{46}$. I. S. Heng ${ }^{46}$ - J. Hennig ${ }^{46}$ - J. Henry ${ }^{117}$.

A. W. Heptonstall ${ }^{1}$ - M. Heurs ${ }^{11,21}$ - S. Hild ${ }^{46}$ - E. Hirose H6 $^{36}$ D. Hoak ${ }^{44}$.

D. Hofman ${ }^{76}$ - K. Holt ${ }^{7}$ - D. E. Holz ${ }^{87}$ - P. Hopkins ${ }^{105}$ - J. Hough ${ }^{46}$.

E. A. Houston ${ }^{46}$. E. J. Howell ${ }^{63}$ - Y. M. Hu ${ }^{11}$. E. A. Huerta ${ }^{118}$.

D. Huet $^{29}$ - B. Hughey ${ }^{112}$ - S. Husa ${ }^{96}$ - S. H. Huttner ${ }^{46}$ - T. Huynh-Dinh ${ }^{7}$.

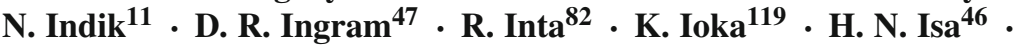
J.-M. Isac ${ }^{71}$ - M. Isi ${ }^{1}$ - T. Isogai ${ }^{13}$ - Y. Itoh It $^{26}$ B. R. Iyer ${ }^{18}$ - K. Izumi ${ }^{47}$.

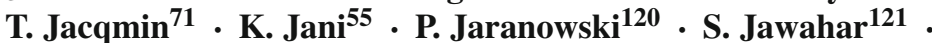

F. Jiménez-Forteza ${ }^{96}$ - W. W. Johnson ${ }^{2}$ - D. I. Jones ${ }^{122}$ - R. Jones ${ }^{46}$.

R. J. G. Jonker ${ }^{12}$ • L. Ju ${ }^{63}$ • J. Junker ${ }^{11}$ - T. Kagawa ${ }^{104}$ - T. Kajita ${ }^{36}$.

M. Kakizaki ${ }^{104}$ - C. V. Kalaghatgi ${ }^{105}$ - V. Kalogera ${ }^{95}$ - M. Kamiizumi ${ }^{36}$. $^{2}$

N. Kanda ${ }^{123}$ - S. Kandhasamy ${ }^{83}$ - S. Kanemura ${ }^{104}$ - M. Kaneyama ${ }^{123}$.

G. Kang ${ }^{89}$ - J. B. Kanner ${ }^{1}$ - S. Karki ${ }^{70}$ - K. S. Karvinen ${ }^{11}$.

M. Kasprzack ${ }^{2}$ - Y. Kataoka ${ }^{35}$ - E. Katsavounidis ${ }^{13}$ - W. Katzman ${ }^{7}$.

S. Kaufer ${ }^{21}$ - T. Kaur ${ }^{63}$ - K. Kawabe ${ }^{47}$ - N. Kawai ${ }^{35}$ - S. Kawamura ${ }^{36}$.

F. Kéfélian ${ }^{65}$ - D. Keitel ${ }^{96}$ - D. B. Kelley ${ }^{45}$ - R. Kennedy ${ }^{100}$ - J. S. Key ${ }^{124}$.

F. Y. Khalili' ${ }^{60}$ - I. Khan ${ }^{15}$ - S. Khan ${ }^{105}$ - Z. Khan99 - E. A. Khazanov ${ }^{125}$.

N. Kijbunchoo ${ }^{47}$ - C. Kim ${ }^{126}$ - H. Kim ${ }^{127}$ - J. C. Kim ${ }^{128}$ - J. Kim ${ }^{129}$.

W. Kim ${ }^{80}$ - Y.-M. Kim ${ }^{130,131}$ - S. J. Kimbrell ${ }^{55}$ - N. Kimura ${ }^{116}$.

E. J. King ${ }^{80}$ - P. J. King ${ }^{47}$ - R. Kirchhoff ${ }^{11}$ - J. S. Kissel ${ }^{47}$ • B. Klein ${ }^{95}$.

L. Kleybolte ${ }^{33}$ - S. Klimenko ${ }^{6}$ - P. Koch ${ }^{11}$ - S. M. Koehlenbeck ${ }^{11}$.

Y. Kojima ${ }^{132}$ - K. Kokeyama ${ }^{36}$ - S. Koley ${ }^{12}$ - K. Komori ${ }^{25}$.

V. Kondrashov ${ }^{1}$ - A. Kontos ${ }^{13}$ - M. Korobko ${ }^{33}$ - W. Z. Korth ${ }^{1}$.

K. Kotake ${ }^{133}$ - I. Kowalska ${ }^{73}$ - D. B. Kozak ${ }^{1}$ - C. Krämer ${ }^{11}$ - V. Kringel ${ }^{11}$.

B. Krishnan ${ }^{11}$ - A. Królak ${ }^{134,135}$ - G. Kuehn ${ }^{11}$ - P. Kumar ${ }^{107}$.

Rahul Kumar $^{116}$ - Rakesh Kumar99 - L. Kuo ${ }^{85}$ - K. Kuroda ${ }^{36}$.

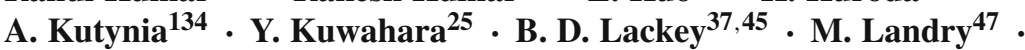

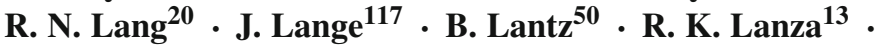

A. Lartaux-Vollard ${ }^{29}$ - P. D. Lasky ${ }^{136}$ - M. Laxen ${ }^{7}$ - A. Lazzarini ${ }^{1}$.

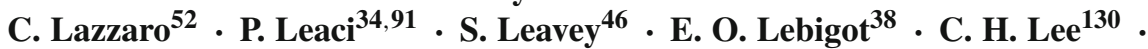

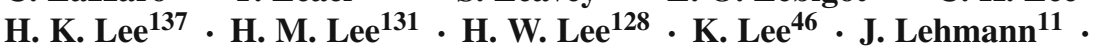

A. Lenon ${ }^{40,41}$ - M. Leonardi ${ }^{102,103}$ - J. R. Leong11 • N. Leroy ${ }^{29}$.

N. Letendre ${ }^{8}$ - Y. Levin ${ }^{136}$. T. G. F. Li ${ }^{138}$ - A. Libson ${ }^{13}$.

T. B. Littenberg ${ }^{139}$ - J. Liu ${ }^{63}$ - N. A. Lockerbie ${ }^{121}$ - A. L. Lombardi ${ }^{55}$.

L. T. London ${ }^{105}$ - J. E. Lord ${ }^{45}$ - M. Lorenzini ${ }^{15,16}$ - V. Loriette ${ }^{140}$.

M. Lormand ${ }^{7}$ - G. Losurdo 23 - J. D. Lough ${ }^{11,21}$ - C. O. Lousto117 .

G. Lovelace ${ }^{28}$. H. Lück11,21 - A. P. Lundgren'11 - R. Lynch'13 . Y. Ma ${ }^{62}$.

S. Macfoy $^{61}$ - B. Machenschalk ${ }^{11}$ - M. MacInnis ${ }^{13}$ - D. M. Macleod ${ }^{2}$.

F. Magaña-Sandoval ${ }^{45}$ - E. Majorana ${ }^{34}$. I. Maksimovic Mo $^{140}$.

V. Malvezzi 16,32 - N. Man ${ }^{65}$ - V. Mandic ${ }^{141}$ - V. Mangano46 S. Mano142

G. L. Mansell ${ }^{24}$ - M. Manske Mo $^{20}$ M. Mantovani ${ }^{44}$ - F. Marchesoni ${ }^{43,143}$.

M. Marchio19 . F. Marion ${ }^{8}$ - S. Márka ${ }^{49}$ - Z. Márka49 .

A. S. Markosyan ${ }^{50}$ - E. Maros ${ }^{1}$. F. Martelli ${ }^{68,69}$ - L. Martellini65 . 


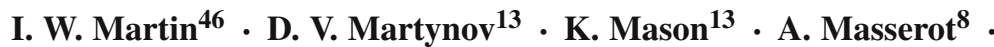
T. J. Massinger ${ }^{1}$ - M. Masso-Reid ${ }^{46}$ - S. Mastrogiovanni ${ }^{34,91}$.

F. Matichard ${ }^{1,13}$. L. Matone M9 $^{4}$ N. Matsumoto ${ }^{144}$. F. Matsushima ${ }^{104}$.

N. Mavalvala ${ }^{13}$ - N. Mazumder ${ }^{67}$ - R. McCarthy ${ }^{47}$ - D. E. McClelland ${ }^{24}$. $^{2}$

S. McCormick ${ }^{7}$ - C. McGrath ${ }^{20}$ - S. C. McGuire ${ }^{145}$ - G. McIntyre ${ }^{1}$.

J. McIver ${ }^{1}$ - D. J. McManus 24 . T. McRae ${ }^{24}$. S. T. McWilliams 40,41 .

D. Meacher ${ }^{65,84}$. G. D. Meadors ${ }^{11,37}$ - J. Meidam ${ }^{12}$ - A. Melatos ${ }^{146}$.

G. Mendell ${ }^{47}$ - D. Mendoza-Gandara ${ }^{11}$ - R. A. Mercer ${ }^{20}$ - E. L. Merilh ${ }^{47}$.

M. Merzougui ${ }^{65}$ - S. Meshkov ${ }^{1}$ - C. Messenger ${ }^{46}$ - C. Messick $^{84}$.

R. Metzdorff ${ }^{71}$ - P. M. Meyers ${ }^{141}$ - F. Mezzani ${ }^{34,91}$ - H. Miao ${ }^{56}$.

C. Michel $^{76}$ - Y. Michimura ${ }^{25}$ - H. Middleton ${ }^{56}$ - E. E. Mikhailov ${ }^{147}$.

L. Milano ${ }^{5,78}$ - A. L. Miller6,34,91 - A. Miller ${ }^{95}$ - B. B. Miller ${ }^{95}$ - J. Miller ${ }^{13}$.

M. Millhouse ${ }^{94}$ - Y. Minenkov ${ }^{16}$ - J. Ming ${ }^{37}$ - S. Mirshekari ${ }^{148}$.

C. Mishra ${ }^{18}$ - V. P. Mitrofanov ${ }^{60}$. G. Mitselmakher6 ${ }^{6}$ R. Mittleman $^{13}$.

O. Miyakawa ${ }^{36}$ - A. Miyamoto Min $^{123}$. T. Miyamoto ${ }^{36}$. S. Miyoki ${ }^{36}$.

A. Moggi $^{23}$ - M. Mohan ${ }^{44}$ - S. R. P. Mohapatra ${ }^{13}$ - M. Montani ${ }^{68,69}$.

B. C. Moore ${ }^{106}$ - C. J. Moore ${ }^{90}$. D. Moraru ${ }^{47}$. G. Moreno47 - W. Morii ${ }^{149}$.

S. Morisaki ${ }^{26}$ - Y. Moriwaki ${ }^{104}$ - S. R. Morriss ${ }^{97}$ - B. Mours ${ }^{8}$.

C. M. Mow-Lowry ${ }^{56}$. G. Mueller6 - A. W. Muir ${ }^{105}$.

Arunava Mukherjee $^{18}$ - D. Mukherjee ${ }^{20}$ - S. Mukherjee ${ }^{97}$ - N. Mukund ${ }^{17}$.

A. Mullavey ${ }^{7}$ - J. Munch ${ }^{80}$ - E. A. M. Muniz ${ }^{28}$ - P. G. Murray ${ }^{46}$.

A. Mytidis ${ }^{6}$ - S. Nagano ${ }^{150}$ - K. Nakamura ${ }^{19}$ - T. Nakamura ${ }^{151}$.

H. Nakano ${ }^{151}$ - Masaya Nakano104 Masayuki Nakano ${ }^{36}$ - K. Nakao ${ }^{123}$.

K. Napier ${ }^{55}$ - I. Nardecchia ${ }^{16,32}$ - T. Narikawa ${ }^{123}$ - L. Naticchioni ${ }^{34,91}$.

G. Nelemans ${ }^{12,64}$ - T. J. N. Nelson ${ }^{7}$ - M. Neri ${ }^{57,58}$ - M. Nery ${ }^{11}$.

A. Neunzert ${ }^{115}$ - J. M. Newport ${ }^{3}$ - G. Newton ${ }^{\$ 46}$ - T. T. Nguyen ${ }^{24}$.

W.-T. Ni ${ }^{152,153}$ - A. B. Nielsen ${ }^{11}$ - S. Nissanke $\mathrm{N}^{12,64}$ - A. Nitz ${ }^{11}$ - A. Noack ${ }^{11}$.

F. Nocera ${ }^{44}$. D. Nolting ${ }^{7}$ - M. E. N. Normandin ${ }^{97}$ - L. K. Nuttall ${ }^{45}$.

J. Oberling ${ }^{47}$ • E. Ochsner ${ }^{20}$ - E. Oelker ${ }^{13}$ - G. H. Ogin ${ }^{154}$ • J. J. Oh ${ }^{127}$.

S. H. Oh ${ }^{127}$ - M. Ohashi" ${ }^{36}$ N. Ohishi' ${ }^{19}$ M. Ohkawa ${ }^{155}$ - F. Ohme Oh,105 $^{12}$

K. Okutomi ${ }^{156}$ - M. Oliver96 - K. Ono ${ }^{36}$. Y. Ono104 . K. Oohara ${ }^{157}$.

P. Oppermann ${ }^{11}$ - Richard J. Oram ${ }^{7}$ - B. O'Reilly ${ }^{7}$ - R. O'Shaughnessy ${ }^{117}$.

D. J. Ottaway ${ }^{80}$ - H. Overmier ${ }^{7}$ - B. J. Owen ${ }^{82}$ - A. E. Pace ${ }^{84}$ - J. Page ${ }^{139}$.

A. Pai ${ }^{110}$ - S. A. Pai ${ }^{59}$ - J. R. Palamos ${ }^{70}$ - O. Palashov ${ }^{125}$ - C. Palomba ${ }^{34}$.

A. Pal-Singh ${ }^{33}$ - H. Pan ${ }^{85}$. C. Pankow ${ }^{95}$. F. Pannarale ${ }^{105}$ - B. C. Pant $^{59}$.

F. Paoletti ${ }^{23,44}$ - A. Paoli ${ }^{44}$ - M. A. Papa ${ }^{11,20,37}$. H. R. Paris ${ }^{50}$.

W. Parker ${ }^{7}$ - D. Pascucci ${ }^{46}$ - A. Pasqualetti ${ }^{44}$ - R. Passaquieti ${ }^{22,23}$.

D. Passuello ${ }^{23}$ - B. Patricelli ${ }^{22,23}$ - B. L. Pearlstone P6 $^{46}$ Medraza $^{1}$.

R. Pedurand ${ }^{76,158}$ - L. Pekowsky ${ }^{45}$ - A. Pele ${ }^{7}$ - F. E. Peña Arellano ${ }^{19}$.

S. Penn ${ }^{159}$ - C. J. Perez ${ }^{47}$ - A. Perreca ${ }^{1}$ - L. M. Perri ${ }^{95}$ - H. P. Pfeiffer ${ }^{107}$.

M. Phelps ${ }^{46}$ - O. J. Piccinni ${ }^{34,91}$ - M. Pichot ${ }^{65}$ - F. Piergiovanni68,69 ${ }^{\text {. }}$

V. Pierro 9,10 - G. Pillant ${ }^{44}$ - L. Pinard ${ }^{76}$ - I. M. Pinto9,10 - M. Pitkin ${ }^{46}$.

M. Poe ${ }^{20}$ - R. Poggiani'22,23 - P. Popolizio ${ }^{44}$ - A. Post ${ }^{11}$ - J. Powell ${ }^{46}$.

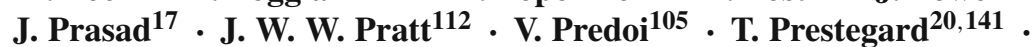

M. Prijatelj11,44 . M. Principe ${ }^{9,10}$. S. Privitera ${ }^{37}$. G. A. Prodi ${ }^{102,103}$.

L. G. Prokhorov ${ }^{60}$ - O. Puncken ${ }^{11}$ - M. Punturo ${ }^{43}$ - P. Puppo ${ }^{34}$. 
M. Pürrer ${ }^{37}$ - H. Qi ${ }^{20}$ - J. Qin ${ }^{63}$ - S. Qiu ${ }^{136}$ - V. Quetschke ${ }^{97}$.

E. A. Quintero ${ }^{1}$ - R. Quitzow-James ${ }^{70}$ - F. J. Raab ${ }^{47}$ - D. S. Rabeling ${ }^{24}$.

H. Radkins ${ }^{47}$ - P. Raffai ${ }^{53}$ - S. Raja ${ }^{59}$ - C. Rajan $^{59}$ - M. Rakhmanov ${ }^{97}$.

P. Rapagnani' ${ }^{34,91}$ - V. Raymond ${ }^{37}$ - M. $\operatorname{Razzano}^{22,23} \cdot$ V. $\operatorname{Re}^{32} \cdot \mathrm{J}^{2} \operatorname{Read}^{28}$.

T. Regimbau ${ }^{65}$ - L. $\operatorname{Re}^{58}$. S. Reid ${ }^{61}$ - D. H. Reitze $e^{1,6}$ - H. Rew ${ }^{147}$.

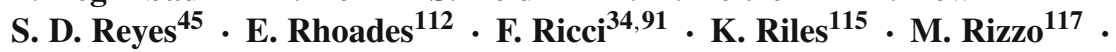

N. A. Robertson ${ }^{1,46}$ - R. Robie R6 $^{46}$. Robinet ${ }^{29}$ - A. Rocchi ${ }^{16}$ - L. Rolland 8 .

J. G. Rollins ${ }^{1}$ - V. J. Roma ${ }^{70}$ - R. Romano ${ }^{4,5}$ - J. H. Romie ${ }^{7}$.

D. Rosińska ${ }^{54,160}$ - S. $\operatorname{Rowan}^{46}$ - A. Rüdiger ${ }^{11}$ - P. Ruggi ${ }^{44}$ - K. Ryan ${ }^{47}$.

S. Sachdev ${ }^{1}$ - T. Sadecki ${ }^{47}$ - L. Sadeghian ${ }^{20}$ - N. Sago ${ }^{161}$ - M. Saijo ${ }^{162}$.

Y. Saito ${ }^{36}$ - K. Sakai ${ }^{163}$ - M. Sakellariadou ${ }^{164}$ - L. Salconi ${ }^{44}$.

M. Saleem ${ }^{110}$ - F. Salemi ${ }^{11}$ - A. Samajdar ${ }^{165}$ - L. Sammut ${ }^{136}$.

L. M. Sampson ${ }^{95}$ - E. J. Sanchez ${ }^{1}$ - V. Sandberg ${ }^{47}$ - J. R. Sanders ${ }^{45}$.

Y. Sasaki ${ }^{166}$ - B. Sassolas ${ }^{76}$ - B. S. Sathyaprakash ${ }^{84,105}$ - S. Sato ${ }^{167}$.

T. Sato ${ }^{155}$ - P. R. Saulson ${ }^{45}$ - O. Sauter ${ }^{115}$ - R. L. Savage S7 $^{4}$

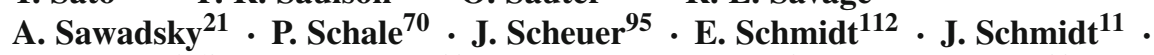

P. Schmidt ${ }^{1,62}$ - R. Schnabel ${ }^{33}$ - R. M. S. Schofield ${ }^{70}$ - A. Schönbeck ${ }^{33}$.

E. Schreiber ${ }^{11}$. D. Schuette S1,21 $^{11}$ B. F. Schutz ${ }^{37,105}$. S. G. Schwalbe ${ }^{112}$.

J. Scott ${ }^{46}$ - S. M. Scott ${ }^{24}$ - T. Sekiguchi ${ }^{36}$ - Y. Sekiguchi ${ }^{168}$ - D. Sellers ${ }^{7}$.

A. S. Sengupta ${ }^{169}$ - D. Sentenac ${ }^{44}$. V. Sequino ${ }^{16,32}$ - A. Sergeev ${ }^{125}$.

Y. Setyawati ${ }^{12,64}$ - D. A. Shaddock ${ }^{24}$ - T. J. Shaffer ${ }^{47}$ - M. S. Shahriar ${ }^{95}$.

B. Shapiro ${ }^{50}$ - P. Shawhan ${ }^{75}$ - A. Sheperd ${ }^{20}$ - M. Shibata ${ }^{119}$.

Y. Shikano ${ }^{170,171}$. T. Shimoda ${ }^{25}$ - A. Shoda ${ }^{19}$. D. H. Shoemaker ${ }^{13}$.

D. M. Shoemaker ${ }^{55}$ - K. Siellez ${ }^{55}$. X. Siemens ${ }^{20}$. M. Sieniawska ${ }^{54}$.

D. Sigg ${ }^{47}$ - A. D. Silva ${ }^{14}$ - A. Singer ${ }^{1}$ - L. P. Singer ${ }^{79}$ - A. Singh 11,21,37 .

R. Singh ${ }^{2}$ - A. Singhal ${ }^{15}$ - A. M. Sintes ${ }^{96}$ - B. J. J. Slagmolen ${ }^{24}$ - B. Smith ${ }^{7}$. J. R. Smith ${ }^{28}$ - R. J. E. Smith ${ }^{1}$ - K. Somiya ${ }^{35}$ - E. J. Son ${ }^{127}$ • B. Sorazu ${ }^{46}$.

F. Sorrentino ${ }^{58}$. T. Souradeep ${ }^{17}$. A. P. Spencer ${ }^{46}$. A. K. Srivastava ${ }^{99}$.

A. Staley ${ }^{49}$ - M. Steinke ${ }^{11}$ - J. Steinlechner ${ }^{46}$ - S. Steinlechner ${ }^{33,46}$.

D. Steinmeyer ${ }^{11,21}$ - B. C. Stephens ${ }^{20}$ - S. P. Stevenson ${ }^{56}$ - R. Stone ${ }^{97}$.

K. A. Strain 46 - N. Straniero ${ }^{76}$ - G. Stratta ${ }^{68,69}$ - S. E. Strigin 60 .

R. Sturani ${ }^{148}$. A. L. Stuver ${ }^{7}$. Y. Sugimoto ${ }^{104}$. T. Z. Summerscales ${ }^{172}$.

L. Sun ${ }^{146}$ - S. Sunil99 - P. J. Sutton ${ }^{105}$ - T. Suzuki116 - B. L. Swinkels ${ }^{44}$.

M. J. Szczepańczyk ${ }^{112}$ - M. Tacca ${ }^{38}$ - H. Tagoshi ${ }^{123}$ - S. Takada ${ }^{173}$.

H. Takahashi' ${ }^{166}$ - R. Takahashi' ${ }^{19}$ - A. Takamori' ${ }^{27}$. D. Talukder ${ }^{70}$.

H. Tanaka ${ }^{36}$ - K. Tanaka $\mathbf{T}^{123}$ - T. Tanaka ${ }^{151}$ - D. B. Tanner ${ }^{6}$ - M. Tápai ${ }^{111}$.

A. Taracchini ${ }^{37}$ - D. Tatsumi ${ }^{19}$ - R. Taylor ${ }^{1}$. S. Telada ${ }^{174}$. T. Theeg ${ }^{11}$.

E. G. Thomas ${ }^{56}$ - M. Thomas $^{7}$ - P. Thomas ${ }^{47}$ - K. A. Thorne ${ }^{7}$.

E. Thrane ${ }^{136}$ - T. Tippens ${ }^{55}$-S. Tiwari ${ }^{15,103}$ - V. Tiwari ${ }^{105}$.

K. V. Tokmakov ${ }^{121}$ - K. Toland ${ }^{46}$ - T. Tomaru ${ }^{116}$ - C. Tomlinson ${ }^{100}$.

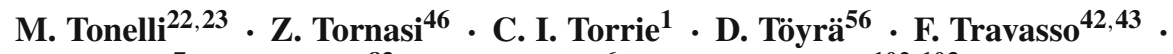

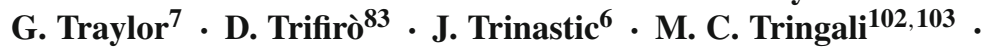

L. Trozzo ${ }^{23,175}$ - M. Tse ${ }^{13}$ - R. Tso ${ }^{1}$ - K. Tsubono ${ }^{25}$ - T. Tsuzuki ${ }^{19}$.

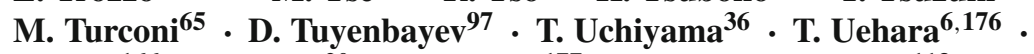

S. Ueki ${ }^{166}$ - K. Ueno ${ }^{20}$ - D. Ugolini ${ }^{177}$ - C. S. Unnikrishnan ${ }^{113}$.

A. L. Urban ${ }^{1}$ - T. Ushiba ${ }^{25}$ - S. A. Usman ${ }^{105}$ - H. Vahlbruch ${ }^{21}$. 
G. Vajente $^{1}$ - G. Valdes $^{97}$ - N. van Bakel ${ }^{12}$ - M. van Beuzekom ${ }^{12}$. J. F. J. van den Brand ${ }^{12,74}$. C. Van Den Broeck ${ }^{12}$ - D. C. Vander-Hyde ${ }^{45}$. L. van der Schaaf ${ }^{12}$ - J. V. van Heijningen ${ }^{12}$ - M. H. P. M. van Putten ${ }^{178}$.

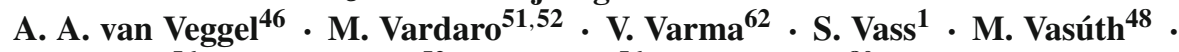

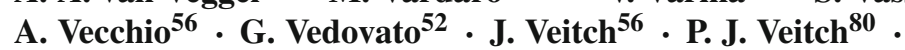

K. Venkateswara ${ }^{179}$ - G. Venugopalan ${ }^{1}$ - D. Verkindt ${ }^{8}$. F. Vetrano V8,69 $^{68,}$

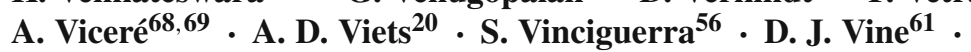

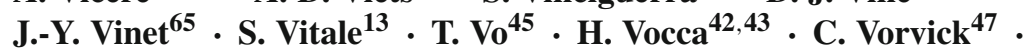
D. V. Voss ${ }^{6}$ - W. D. Vousden ${ }^{56}$ - S. P. Vyatchanin ${ }^{60}$ - A. R. Wade $^{1}$. L. E. Wade ${ }^{88}$ - M. Wade W8 $^{88}$ T. Wakamatsu ${ }^{157}$ - M. Walker ${ }^{2}$. L. Wallace ${ }^{1}$. S. Walsh ${ }^{11,37}$ - G. Wang ${ }^{15,69}$ - H. Wang ${ }^{56}$ - M. Wang ${ }^{56}$ - Y. Wang ${ }^{63}$. R. L. Ward $^{24}$ - J. Warner ${ }^{47}$ - M. Was ${ }^{8}$ - J. Watchi ${ }^{92}$ - B. Weaver ${ }^{47}$. L.-W. Wei ${ }^{65}$ - M. Weinert ${ }^{11}$ - A. J. Weinstein ${ }^{1}$ - R. Weiss ${ }^{13}$ - L. Wen ${ }^{63}$. P. Weßels ${ }^{11}$. T. Westphal ${ }^{11}$. K. Wette ${ }^{11}$. J. T. Whelan ${ }^{117}$. B. F. Whiting6 ${ }^{6}$ C. Whittle ${ }^{136}$ - D. Williams ${ }^{46}$. R. D. Williams ${ }^{1}$ - A. R. Williamson ${ }^{105}$. J. L. Willis ${ }^{180}$ - B. Willke11,21 - M. H. Wimmer11,21 - W. Winkler ${ }^{11}$. C. C. Wipf ${ }^{1}$ - H. Wittel ${ }^{11,21}$ - G. Woan ${ }^{46}$ - J. Woehler ${ }^{11}$ - J. Worden ${ }^{47}$. J. L. Wright ${ }^{46}$ - D. S. Wu ${ }^{11}$ - G. Wu ${ }^{7}$ - W. Yam ${ }^{13}$ - H. Yamamoto ${ }^{1}$. K. Yamamoto ${ }^{36}$ - T. Yamamoto ${ }^{36}$ - C. C. Yancey $^{75}$ - K. Yano ${ }^{35}$. M. J. Yap ${ }^{24}$ - J. Yokoyama ${ }^{26}$ - T. Yokozawa ${ }^{123}$ - T. H. Yoon ${ }^{181} \cdot$ Hang Yu$^{13}$. Haocun Yu' ${ }^{13}$ - H. Yuzurihara ${ }^{123}$ - M. Yvert ${ }^{8}$ - A. Zadrożny ${ }^{134}$. L. Zangrando ${ }^{52} \cdot$ M. Zanolin ${ }^{112}$ - S. Zeidler ${ }^{19}$ - J.-P. Zendri ${ }^{52} \cdot$ M. Zevin ${ }^{95}$. L. Zhang ${ }^{1}$ - M. Zhang ${ }^{147}$ - T. Zhang ${ }^{46}$ - Y. Zhang ${ }^{117}$ - C. Zhao ${ }^{63}$. M. Zhou' ${ }^{95}$ Z. Zhou' ${ }^{95}$. S. J. Zhu ${ }^{11,37}$ - X. J. Zhu ${ }^{63}$ - M. E. Zucker ${ }^{1,13}$. J. Zweizig ${ }^{1}$

\section{* Deceased, March 2016 \\ $\sharp$ Deceased, March 2017 \\ $\dagger$ Deceased, February 2017 \\ $\ddagger$ Deceased, December 2016}

\footnotetext{
1 LIGO, California Institute of Technology, Pasadena, CA 91125, USA

2 Louisiana State University, Baton Rouge, LA 70803, USA

3 American University, Washington, DC 20016, USA

4 Università di Salerno, Fisciano I-84084, Salerno, Italy

5 INFN, Sezione di Napoli, Complesso Universitario di Monte S.Angelo, I-80126 Napoli, Italy

6 University of Florida, Gainesville, FL 32611, USA

7 LIGO Livingston Observatory, Livingston, LA 70754, USA

8 Laboratoire d'Annecy-le-Vieux de Physique des Particules (LAPP), Université Savoie Mont Blanc, CNRS/IN2P3, F-74941 Annecy-le-Vieux, France

9 University of Sannio at Benevento, I-82100 Benevento, Italy

10 INFN, Sezione di Napoli, I-80100 Napoli, Italy
} 
11 Albert-Einstein-Institut, Max-Planck-Institut für Gravitationsphysik, D-30167 Hannover, Germany

12 Nikhef, Science Park, 1098, XG Amsterdam, The Netherlands

13 LIGO, Massachusetts Institute of Technology, Cambridge, MA 02139, USA

14 Instituto Nacional de Pesquisas Espaciais, 12227-010 São José dos Campos, São Paulo, Brazil

15 INFN, Gran Sasso Science Institute, I-67100 L'Aquila, Italy

16 INFN, Sezione di Roma Tor Vergata, I-00133 Roma, Italy

17 Inter-University Centre for Astronomy and Astrophysics, Pune 411007, India

18 International Centre for Theoretical Sciences, Tata Institute of Fundamental Research, Bengaluru 560089, India

19 National Astronomical Observatory of Japan, 2-21-1, Ohsawa, Mitaka-shi, Tokyo 181-8588, Japan

20 University of Wisconsin-Milwaukee, Milwaukee, Wisconsin 53201, USA

21 Leibniz Universität Hannover, D-30167 Hannover, Germany

22 Università di Pisa, I-56127 Pisa, Italy

23 INFN, Sezione di Pisa, I-56127 Pisa, Italy

24 Australian National University, Canberra, Australian Capital Territory 0200, Australia

25 The University of Tokyo, Department of Physics, 7-3-1, Hongo, Bunkyo-ku, Tokyo 113-0033, Japan

26 The University of Tokyo, Research Center for the Early Universe, 7-3-1, Hongo, Bunkyo-ku, Tokyo 113-0033, Japan

27 The University of Tokyo, Earthquake Research Institute, 1-1-1, Yayoi, Bunkyo-ku, Tokyo 113-0032, Japan

28 California State University Fullerton, Fullerton, CA 92831, USA

29 LAL, Univ. Paris-Sud, CNRS/IN2P3, Université Paris-Saclay, F-91898 Orsay, France

30 Chennai Mathematical Institute, Chennai 603103, India

31 Hirosaki University, Department of Advanced Physics, 3, Bunkyo-cho, Hirosaki-shi, Aomori 036-8561, Japan

32 Università di Roma Tor Vergata, I-00133 Roma, Italy

33 Universität Hamburg, D-22761 Hamburg, Germany

34 INFN, Sezione di Roma, I-00185 Roma, Italy

35 Tokyo Institute of Technology, Graduate School of Science and Technology, 2-12-1, Ookayama, Meguro-ku, Tokyo 152-8551, Japan

36 The University of Tokyo, Institute for Cosmic Ray Research, Higashi-Mozumi 238, Kamioka-cho, Hida-shi, Gifu 506-1205, Japan

37 Albert-Einstein-Institut, Max-Planck-Institut für Gravitationsphysik, D-14476 Potsdam-Golm, Germany

38 APC, AstroParticule et Cosmologie, Université Paris Diderot, CNRS/IN2P3, CEA/Irfu, Observatoire de Paris, Sorbonne Paris Cité F-75205, Paris Cedex 13, France

39 Osaka University, Graduate School of Science, Physics, 1-1, Machikaneyama-cho, Toyonaka-shi, Osaka 560-0043, Japan

40 West Virginia University, Morgantown, WV 26506, USA 
41 Center for Gravitational Waves and Cosmology, West Virginia University, Morgantown, WV 26505, USA

42 Università di Perugia, I-06123 Perugia, Italy

43 INFN, Sezione di Perugia, I-06123 Perugia, Italy

44 European Gravitational Observatory (EGO), I-56021 Cascina, Pisa, Italy

45 Syracuse University, Syracuse, NY 13244, USA

46 SUPA, University of Glasgow, Glasgow G12 8QQ, United Kingdom

47 LIGO Hanford Observatory, Richland, WA 99352, USA

Wigner RCP, RMKI, H-1121 Budapest, Konkoly Thege Miklós út 29-33, , Hungary

Columbia University, New York, NY 10027, USA

50 Stanford University, Stanford, CA 94305, USA

51 Università di Padova, Dipartimento di Fisica e Astronomia, I-35131 Padova, Italy

INFN, Sezione di Padova, I-35131 Padova, Italy

MTA Eötvös University, "Lendulet” Astrophysics Research Group, Budapest 1117, Hungary

Center for Relativistic Astrophysics and School of Physics, Georgia Institute of Technology, Atlanta, GA 30332, USA

University of Birmingham, Birmingham B15 2TT, United Kingdom

Università degli Studi di Genova, I-16146 Genova, Italy

INFN, Sezione di Genova, I-16146 Genova, Italy

RRCAT, Indore, MP 452013, India SUPA, University of the West of Scotland, Paisley PA1 2BE, United Kingdom University of Western Australia, Crawley, Western Australia 6009, Australia Nijmegen, The Netherlands

Artemis, Université Côte d'Azur, CNRS, Observatoire Côte d'Azur, CS 34229, F-06304 Nice Cedex 4, France Washington State University, Pullman, WA 99164, USA Università degli Studi di Urbino 'Carlo Bo', I-61029 Urbino, Italy INFN, Sezione di Firenze, I-50019 Sesto Fiorentino, Firenze, Italy University of Oregon, Eugene, OR 97403, USA

71 Laboratoire Kastler Brossel, UPMC-Sorbonne Universités, CNRS, ENS-PSL Research University, Collège de France, F-75005 Paris, France 


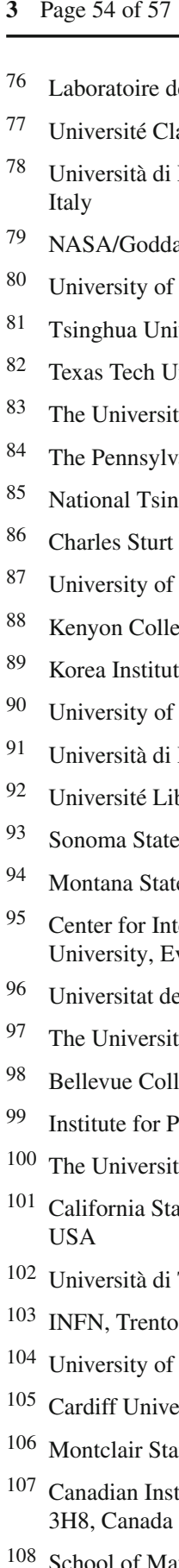

110 IISER-TVM, CET Campus, Trivandrum Kerala 695016, India 
111 University of Szeged, Dóm tér 9, Szeged 6720, Hungary

112 Embry-Riddle Aeronautical University, Prescott, AZ 86301, USA

113 Tata Institute of Fundamental Research, Mumbai 400005, India

114 INAF, Osservatorio Astronomico di Capodimonte, I-80131 Napoli, Italy

115 University of Michigan, Ann Arbor, MI 48109, USA

116 High Energy Accelerator Research Organization, 1-1, Oho, Tsukuba-shi, Ibaraki 305-0801, Japan

117 Rochester Institute of Technology, Rochester, NY 14623, USA

118 NCSA, University of Illinois at Urbana-Champaign, Urbana, IL 61801, USA

119 Center for Gravitational Physics, Yukawa Institute for Theoretical Physics, Kyoto University, Kyoto 606-8502, Japan

120 University of Białystok, 15-424 Białystok, Poland

121 SUPA, University of Strathclyde, Glasgow G1 1XQ, United Kingdom

122 University of Southampton, Southampton SO17 1BJ, United Kingdom

123 Osaka City University, Department of Physics, 3-3-138, Sugimoto-cho, Sumiyosi-ku, Osaka-shi, Osaka 558-8585, Japan

124 University of Washington Bothell, 18115 Campus Way NE, Bothell, WA 98011, USA

125 Institute of Applied Physics, Nizhny Novgorod 603950, Russia

126 Korea Astronomy and Space Science Institute (KASI), 776, Daedeokdae-ro, Yuseong-gu, Daejeon 34055, Republic of Korea

127 National Institute for Mathematical Sciences, Daejeon 34047, Korea

128 Inje University, 197 Inje-ro, Gimhae-si 50834, Korea

129 Myongji University, Yongin 449-728, Korea

130 Pusan National University, Busan 609-735, Korea

131 Seoul National University, Seoul 151-742, Korea

132 Hiroshima University, Department of Physical Science, 1-3-1, Kagamiyama, Higashihiroshima-shi, Hiroshima 739-8526, Japan

133 Department of Applied Physics, Fukuoka University, Fukuoka, Jonan, Nanakuma 814-0180, Japan

134 NCBJ, 05-400 Świerk-Otwock, Poland

135 Institute of Mathematics, Polish Academy of Sciences, 00656 Warsaw, Poland

136 The School of Physics \& Astronomy, Monash University, Clayton 3800, Victoria, Australia

137 Hanyang University, Seoul 133-791, Korea

138 The Chinese University of Hong Kong, Shatin, NT, Hong Kong

139 University of Alabama in Huntsville, Huntsville, AL 35899, USA

140 ESPCI, CNRS, F-75005 Paris, France

141 University of Minnesota, Minneapolis, MN 55455, USA

142 The Institute of Statistical Mathematics, Department of Mathematical Analysis and Statistical Inference, 10-3 Midori-cho, Tachikawa, Tokyo 190-8562, Japan

143 Università di Camerino, Dipartimento di Fisica, I-62032 Camerino, Italy

144 Tohoku University, Sendai, Miyagi 982-0826, Japan 
145 Southern University and A\&M College, Baton Rouge, LA 70813, USA

146 The University of Melbourne, Parkville, Victoria 3010, Australia

147 College of William and Mary, Williamsburg, VA 23187, USA

148 Instituto de Física Teórica, University Estadual Paulista/ICTP South American Institute for Fundamental Research, São Paulo, SP 01140-070, Brazil

149 The Kyoto University, Disaster Prevention Research Institute, Gokasho, Uji, Kyoto 611-0011, Japan

150 National Institute of Information and Communications Technology, The Applied Electromagnetic Research Institute , 4-2-1, Nukuikita-machi, Koganei-shi, Tokyo 184-8795, Japan

151 Kyoto University, Department of Physics, Astronomy, Oiwake-cho, KitaShirakawa, Sakyou-ku, Kyoto-shi, Kyoto 606-8502, Japan

152 National Tsing Hua University, Department of Physics, No. 101, Section 2, Kuang-Fu Road, Hsinchu, Taiwan 30013, ROC

153 University of Shanghai for Science and Technology, School of Optical-Electrical and Computer Engineering, 516, Jun Gong Rd, Shanghai 200093, P. R. China

154 Whitman College, 345 Boyer Avenue, Walla Walla, WA 99362, USA

155 Niigata University, Faculty of Engineering, 8050, Ikarashi-2-no-cho, Nishi-ku, Niigata-shi, Niigata 950-2181, Japan

156 Sokendai (The Graduate University for Advanced Studies), 2-21-1, Ohsawa, Mitaka-shi, Tokyo 181-8588, Japan

157 Niigata University, Graduate School of Science and Technology, 8050, Ikarashi-2-no-cho, Nishi-ku, Niigata-shi, Niigata 950-2181, Japan

158 Université de Lyon, F-69361 Lyon, France

159 Hobart and William Smith Colleges, Geneva, NY 14456, USA

160 Janusz Gil Institute of Astronomy, University of Zielona Góra, 65-265 Zielona Góra, Poland

161 Kyushu University, Faculty of Arts and Science, 744, Motooka, Nishi-ku, Fukuoka 819-0395, Japan

162 Waseda University, Department of Physics, 3-4-1, Okubo, Shinjuku, Tokyo 169-8555, Japan

163 Nagaoka University of Technology, Department of Information Science and Control Engineering, 1603-1 Kamitomioka, Nagaoka, Niigata 940-2188, Japan

164 King's College London, University of London, London WC2R 2LS, United Kingdom

165 IISER-Kolkata, Mohanpur, West Bengal 741252, India

166 Nagaoka University of Technology, Department of Information \& Management Systems Engineering, 1603-1 Kamitomioka, Nagaoka, Niigata 940-2188, Japan

167 Hosei University, The Graduate School of Science and Engineering, Kajino-cho 3-7-2, Koganei-shi, Tokyo 184-8584, Japan

168 Toho University, Faculty of Science, 2-2-1 Miyama, Funabashi-shi, Chiba, Japan

169 Indian Institute of Technology, Gandhinagar Ahmedabad Gujarat, 382424, India

170 Institute for Molecular Science, National Institutes of Natural Sciences, 38 Nishigo-Naka, Myodaiji, Okazaki 444-8585, Japan

171 Institute for Quantum Studies, Chapman University, 1 University Dr, Orange, CA 92866, USA

172 Andrews University, Berrien Springs, MI 49104, USA 
173 National Institutes of Natural Sciences, The Device Engineering and Applied Physics Research Division, 322-6 Oroshi-cho, Toki city, GIFU Prefecture 509-5292, Japan

174 National Institute of Advanced Industrial Science and Technology, Metrology Institute of Japan, 1-1-1, Umezono, Tsukuba-shi, Ibaraki 305-8568, Japan

175 Università di Siena, I-53100 Siena, Italy

176 National Defense Academy of Japan, Department of Communications Engineering, Hashirimizu 1-10-20, Yokosuka-shi, Kanagawa-Pref 239-8686, Japan

177 Trinity University, San Antonio, TX 78212, USA

178 Physics and Astronomy, Sejong University, 209 Neungdong-ro, Gwangjin-gu 143-747, Seoul, South Korea

179 University of Washington, Seattle, WA 98195, USA

180 Abilene Christian University, Abilene, TX 79699, USA

181 Department of Physics, Korea University, 145, Anam-ro, Seongbuk-gu, Seoul 02841, Korea1 